\title{
A Connected Sum Construction for Complete Minimal Surfaces of Finite Total Curvature
}

\author{
SEONG-DeOG YANG
}

\section{Introduction.}

Background. In the study of minimal surfaces, it is of great value to have a large collection of examples for reference and insight. The purpose of this article is to develop a general procedure for constructing complete minimal surfaces of finite total curvature in $\mathbb{E}^{3}$.

As far as the author knows, most, if not all, of the nontrivial examples of complete minimal surfaces have been found through extensive use of a global version of the Weierstrass representation theorem; one first assumes the existence of a minimal surface with certain properties, makes a good guess at the complex theoretic data, and then determines whether or not the period problem is solved. This method has had great success. (See $[\mathrm{N}],[\mathrm{DHKW}],[\mathrm{HK}]$ for a survey.) But, since this method uses complex data, it is not easy to gain insight into the examples constructed this way. Furthermore, the period problem restricts examples found by this method to those that have a lot of symmetry in general. For instance, it was only in 1980 that the first example of an arbitrary number of catenoidal ends was found $[\mathrm{JM}]$. For these reasons, a more direct and geometric method of constructing complete minimal surfaces of finite total curvature is desirable. In particular, one hopes that a general method will be found to construct complicated ones from simple ones.

In this paper, I provide such a procedure by solving a nonlinear P.D.E. with singular perturbation methods. Kapouleas was the first who applied this technique in order to construct complete embedded minimal surfaces of finite total curvature, by desingularizing the circles of intersection of a collection of catenoids and planes with a common axis [K5]. Here, I prove the following.

The main results. For any integer $I \geq 0$, fix a collection $\mathcal{M}:=\left\{N_{i}\right\}_{i=1}^{I+1}$ of complete, immersed (without any branch points), orientable, minimal 
surfaces in $\mathbb{E}^{3}$ of nonzero finite total curvature. Assume that none of the $N_{i}$ 's has an exceptional bounded Jacobi field (whose meaning will be explained shortly). Then, there exists a very small number $\tau_{0}>0$ depending upon $\mathcal{M}$ such that the following is true:

For any $0<\tau<\tau_{0}$ and any integer $K \geq 0$ with $I+K>0$, let $\mathcal{B}:=\left\{B_{m}\right\}_{m=K+1}^{K+I}$ and $\mathcal{E}:=\left\{E_{m}\right\}_{m=1}^{K}$ be collections of catenoidal necks and catenoidal ends, respectively, of neck sizes $\tau_{m}$ which satisfy $\tau_{m}<\tau$ and $C^{-1} \leq \tau_{i} / \tau_{j} \leq C$ for some constant $\mathrm{C}$ depending only on $\mathcal{M}$. But, if $I=0$, then $\mathcal{B}:=\emptyset$, and if $\mathrm{K}=0$, then $\mathcal{E}:=\emptyset$. Then we can construct a complete, immersed, orientable, minimal surface of finite total curvature by gluing together all the elements of $\mathcal{M} \cup \mathcal{B} \cup \mathcal{E}$ in the following manner:

1. $B_{m}$ serves as a bridge between two elements of $\mathcal{M}$.

2. $E_{m}$ is glued to an element of $\mathcal{M}$.

3. The resulting minimal surface can be thought of as a tree when we consider the $N_{i}$ 's as vertices and the $B_{m}$ 's as edges.

The gluing can occur at any point and on either side of $N_{i}$. When $\tau \rightarrow 0$, the resulting minimal surface converges to the union of $N_{i}$ 's touching at the points where the gluing occurs, and $B_{m}$ 's and $E_{m}$ 's converge to catenoids if they are appropriately scaled.

Recently, there appeared new results in applying gluing techniques for constructing constant mean curvature surfaces in $\mathbb{E}^{3}[\mathrm{MP}][\mathrm{MPP}]$ and minimal hypersurfaces in $\mathbb{E}^{n}$ for $n \geq 4[\mathrm{FP}]$.

About Jacobi fields. Recall that any minimal surface necessarily possesses Jacobi fields which arise from translations, homothetic expansions, rotations, and possibly other Jacobi fields. I shall call any Jacobi field which does not arise from translations exceptional. Refer to $[\mathrm{CR}],[\mathrm{P}],[\mathrm{PR}]$. Unfortunately we need to assume that there are no exceptional bounded Jacobi fields for some technical reasons which will be explained in the outline of the proof. However, the assumption is quite general in the sense that Ejiri \& Kotani and Montiel \& Ros independently proved that it holds true for generic complete (finitely branched) minimal surfaces with 'genus zero' of finite total curvature [EK], [MR]. In particular, the assumption holds true if the branching values of the Gauss map lie in an equator [MR, Corollary 15], which is the case for any complete orientable minimal surface of finite total curvature parameterized on the unit sphere $\mathbb{C} \cup\{\infty\}$ with the Gauss 


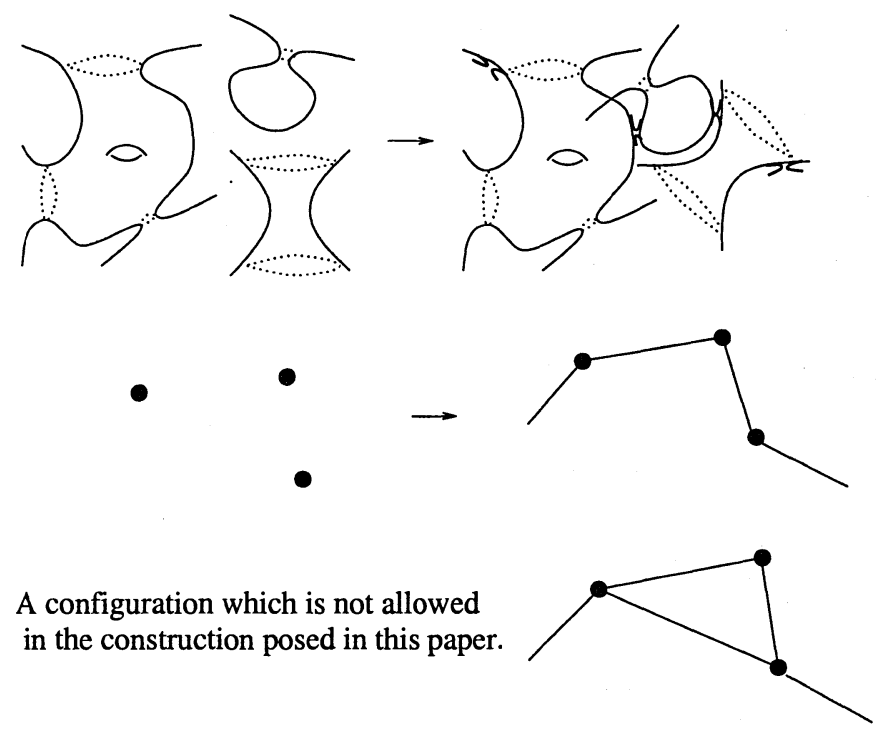

Figure 1.

map $g=\omega^{n}, n \geq 1$. See [DHKW, §3.8] for explicit descriptions of examples of such minimal surfaces, which include catenoids, Enneper's surfaces, Jorge-Meeks n-noids, etc.

On the other hand, there do exist complete minimal surfaces which have exceptional bounded Jacobi fields. For instance, $[\mathrm{EK}],[\mathrm{MR}]$ say that the Jacobi fields which arise from homothetic expansions for flat-ended minimal surfaces (refer to [RT] for such examples), as well as the Jacobi fields which arise from rotations around the axes of the minimal surfaces constructed by Kapouleas [K5], are (nontrivial and) bounded. It would be nice to be able to use those minimal surfaces also as ingredients in the construction posed in this paper.

Outline of the proof. The construction posed in this paper is an application of the gluing techniques developed by Schoen [S2] and Kapouleas [K1-5], in the form they evolved into in [K4]. For the convenience of the reader, we proceed now to provide an outline of the general approach, some of the difficulties in applying them to our case, and the methods by which we overcome them.

First, we construct an approximately minimal surface: 1) fix a collection 
of minimal surfaces with no exceptional bounded Jacobi fields and catenoids of very small neck sizes; 2) remove small disks from the minimal surfaces, remove one or two ends from each of the catenoids; 3 ) arrange them so that the boundary circles of the truncated catenoids fit closely into the holes of the drilled minimal surfaces; 4) then glue them together to obtain a connected, immersed, complete, orientable surface, say $\vec{X}: M \rightarrow \mathbb{E}^{3}$, of finite total curvature. The general approach requires that the mean curvature should be small relative to the neck sizes of the catenoids, and for this purpose we need to pay more than passing attention to choosing appropriately the relative sizes of the holes in the minimal surfaces and the boundary circles of the truncated catenoids.

Then, we look for a perturbation of the surface which is minimal. More specifically, when $\vec{\nu}$ denotes a unit normal vector field of $\mathrm{M}$, we look for a function $\varphi: M \rightarrow \mathbb{R}$ such that $\vec{X}_{\varphi}: M \rightarrow \mathbb{E}^{3}$, given by $\vec{X}_{\varphi}=\vec{X}+\varphi \vec{\nu}$, is minimal. Recall that for a real valued function $\phi$ on $M$, the mean curvature $H_{\phi}$ of $\vec{X}_{\phi}: M \rightarrow \mathbb{E}^{3}$, given by $\vec{X}_{\phi}:=\vec{X}+\phi \vec{\nu}$, can be written as follows:

$$
H_{\phi}=H+\frac{1}{2}\left(\Delta_{g}+|\mathbf{A}|^{2}\right) \phi+\tilde{Q_{\phi}},
$$

where $H$ is the mean curvature function of $\vec{X}(M), \Delta_{g}$ is the Laplacian of $M,|\mathbf{A}|^{2}$ is the square of the length of the second fundamental form, and $\tilde{Q_{\phi}}$ is the nonlinear term. So, our problem is now to find a function $\varphi: M \rightarrow \mathbb{R}$ which solves the minimal surface equation

$$
0=H+\frac{1}{2}\left(\Delta_{g}+|\mathbf{A}|^{2}\right) \varphi+\tilde{Q_{\varphi}}
$$

We find such $\varphi$ by using the Schauder fixed point theorem [GT], and the heuristic method is as follows: let $\phi_{1}$ be a solution of the linearized minimal surface equation, that is

$$
\left(\Delta_{g}+|\mathbf{A}|^{2}\right) \phi_{1}=-2 H .
$$

For each function $\phi$ of $M$ into $\mathbb{R}$, we define $\phi_{2}$ to be a solution to

$$
\left(\Delta_{g}+|\mathbf{A}|^{2}\right) \phi_{2}=-2 \tilde{Q_{\phi}} .
$$

Then, we would have a map $\mathcal{J}$ sending $\phi$ to $\phi_{1}+\phi_{2}$, and we thus see that a fixed point of this map is going to be a solution of (1.2).

The success of our work rests on our ability to construct a solution $\phi_{1}$ to (1.3) such that $\phi_{1}$ is the same size as $H$ and that $\tilde{Q}_{\phi_{1}}$ is much smaller than $\phi_{1}$, from which there are two main issues worthy of mention. 
The first issue is the invertibility of the linearized operator $\Delta_{g}+|\mathbf{A}|^{2}$, i.e., the Jacobi operator. We first compactify $M$ using the Weierstrass representation theorem. The general approach developed in [K1-5] requires us to understand the spectrum of the linearized operator not on the entire $M$ but on appropriate domains, each of which is a standard piece extended by joining pieces. Roughly speaking, a standard piece is a drilled minimal surface or a truncated catenoid, and a joining piece is a neighborhood of the region where the gluing occurs. (Refer to the remark after definition 3.2.2.) The domains overlap on the joining pieces and cover $M$. It turns out that there are small (i.e., indistinguishable from 0 ) Dirichlet eigenvalues for the linearized operator on each domain. So, we solve the linearized equation with the Dirichlet condition on each domain modulo the eigenspace of those small eigenvalues (i.e., modulo the approximate kernel), and correct the local solutions later by the force balancing argument. And, this is exactly where the assumption of no exceptional bounded Jacobi fields plays a role, for the force balancing arguments can take care of the bounded Jacobi fields which arise from translations.

To apply the force balancing argument, we need to perturb an arbitrary end so that a prescribed change of forces is achieved. If the end possesses a nonvanishing force, this can be easily arranged by scaling and rotation. But these do not work for the ends of vanishing forces, for example, the end of Enneper's minimal surface. There appeared a similar situation in [K4], and the "geometric principle" was proposed to help overcome the difficulty there. In the present construction, perturbing the ends of vanishing forces is one of the greatest difficulties, and fortunately, it can be achieved through a study of ends using the Weierstrass representation theorem.

The second issue is the decay property of the local solutions along the joining pieces. Following the general approach, we obtain a global solution $\phi_{1}$ to (1.3) by gluing local solutions and taking care of the error term by iteration. The decay of local solutions ensures the smallness of the error term, hence the success of the iteration, and the smallness of the nonlinear term $\tilde{Q}_{\phi_{1}}$ for $\phi_{1}$. We achieve the required decay by adopting the ideas from $[\mathrm{K} 2,4,5]$ : we first modify the local solutions through the addition of a certain function in order to force the solutions to decay exponentially along joining pieces, and then we correct them by rearranging the relative positions of the drilled minimal surfaces and the truncated catenoids.

Because of the perturbation of ends and the rearrangement of the relative positions, we construct a whole family of approximately minimal surfaces and single out an appropriate one to be perturbed by a fixed point argument in the proof of the main theorem. An elaborate accounts for the general pro- 
cedure for solving the linearized minimal surface equation and the analytic reason for the perturbations are explained in the beginning of section 4, but one needs to get familiar with the notation to be developed in subsections 3.1 and 3.2 .

Outline of the paper. In section 2, I present some results related to the ends of complete minimal surfaces of finite total curvature: using the Weierstrass representation theorem, I classify the ends and study their forces. Then I devise a way to perturb the ends (of vanishing forces) by deforming the Weierstrass data so that prescribed changes in the forces can be achieved. In section 3 , I construct a family of approximately minimal surfaces and develop much of the notation. In section 4, I solve the linearized minimal surface equation (1.3). In section 5 , I solve (1.4) and combine the result with that of section 4 to prove the main theorem.

Acknowledgments. I would like to thank my thesis advisor, Professor N. Kapouleas, for suggesting this problem, and for his constant encouragement and stimulating discussions on this subject. This work was partially supported by a Sloan Doctoral Dissertation Fellowship.

This paper is dedicated to the memory of my father, Ik-Hyun Yang.

\section{Ends of complete minimal surfaces of finite total curvature.}

In this section we use the Weierstrass representation theorem extensively to classify ends of complete minimal surfaces of finite total curvature and to show that any end can be perturbed so that a prescribed small change of forces can be achieved. We need this perturbation of ends to handle the kernel of the linearized operator $\Delta_{g}+|\mathbf{A}|^{2}$. When an end has a nonvanishing force, changing forces could be done easily by simply rotating and resizing the end. But, this idea does not work for ends of vanishing forces, for example the end of Enneper's surface. We perturb such ends by deforming the corresponding Weierstrass data. We start with

Theorem 2.1.1 ([O], [HK] Weierstrass representation theorem). Suppose $N$ is a minimal surface in $\mathbb{E}^{3}, M$ its Riemann surface, $g$ the stereographic projection of its Gauss map. Then, $N$ may be represented, up to a 
A connected sum construction for complete minimal surfaces

translation, by the conformal immersion

$$
\vec{X}=(x, y, z):=\operatorname{Re} \int \Phi,
$$

where

$$
\Phi=\left(\phi_{1}, \phi_{2}, \phi_{3}\right):=\left(\frac{1}{2}\left(g^{-1}-g\right) d h, \frac{i}{2}\left(g^{-1}+g\right) d h, d h\right)
$$

for some holomorphic one-form dh on $M$. When $\vec{X}: M \rightarrow \mathbb{E}^{3}$ is a complete conformal minimal immersion with finite total curvature,

1. $M$ is conformally diffeomorphic to $\bar{M} \backslash\left\{p_{1}, \ldots, p_{r}\right\}$ where $\bar{M}$ is a closed Riemann surface, and $r \geq 1$,

2. $\vec{X}$ is proper,

3. The Gauss map $M \rightarrow \mathbb{S}^{2}$, which is meromorphic on $M$, extends to a meromorphic function on $\bar{M}$; the holomorphic one-form dh extends to a meromorphic one-form on $\bar{M}$,

4. The total curvature is an integer multiple of $4 \pi$ and satisfies

$$
\int_{M} K d A \leq-4 \pi(k+r-1)=2 \pi(\chi(M)-1),
$$

where $k$ is the genus of $\bar{M}, r$ as in 1 , and $\chi(M)$ is the Euler characteristic of $M$.

Conversely, let $M$ be a Riemann surface, $g: M \rightarrow \mathbb{C} \cup\{\infty\}$ a meromorphic function and dh a holomorphic one-form on $M$. Then (2.1) and (2.2) define a conformal minimal mapping of some covering of $M$ into $\mathbb{E}^{3}$, which is regular provided the poles and zeros of $g$ coincide with the zeros of dh with same orders. The mapping $\vec{X}$ is well-defined on $M$ if and only if no component of $\Phi$ in (2.2) has a real period. That is

$$
\operatorname{Period}_{\gamma}(\Phi):=\operatorname{Re} \oint_{\gamma} \Phi=0
$$

for all closed curves $\gamma$ on $M$. 
If we denote by $\mathbf{g}$ the metric inherited from the standard metric of $\mathbb{E}^{3}$, by $\mathbf{A}$ the second fundamental form, then we have from [DHKW][HK]

$$
\begin{gathered}
\mathbf{g}=\frac{1}{2}|\Phi|^{2}=\frac{\left(1+|g|^{2}\right)^{2}}{4|g|^{2}}|d h|^{2}, \\
\mathbf{A}=\operatorname{Re}\left\{\frac{d g}{g} \cdot d h\right\}, \quad|\mathbf{A}|^{2}=-2 K=\frac{32|g|^{2}}{\left(1+|g|^{2}\right)^{4}}\left|\frac{d g}{d h}\right|^{2} .
\end{gathered}
$$

For any $p \in \bar{M}$, we can find a neighborhood $D$ of $p$ such that we may assume

$$
\begin{gathered}
p=0 \in D=D_{\delta^{\prime}}=\left\{\omega \in \mathbb{C}:|\omega|<\delta^{\prime}\right\}, \\
g=\omega^{n}, \quad d h=\sum_{m=k}^{\infty} a_{m} \omega^{m} d \omega
\end{gathered}
$$

for some $\delta^{\prime}>0, a_{k} \neq 0$, and $\mathrm{n}, \mathrm{k} \in \mathbb{Z}$ with $n \geq 1$, by rotating and translating $\vec{X}(M)$ in $\mathbb{E}^{3}$ and reparameterizing $D$ if necessary,

There is an interesting relationship between $n$ and $k$.

Proposition 2.1.2. Assume (2.4). Then, $p$ is a branch point iff $k>n . p$ is a regular point iff $k=n$. ( $p$ is umbilic iff $k=n>1$.) $p$ is a puncture iff $k<n-1$.

Proof. First of all, if $k=n-1$, then Res $s_{0} g d h=0$ and $\operatorname{Res}_{0} g^{-1} d h=a_{k} \neq$ 0 . Hence, $\operatorname{Res}_{0} \phi_{1}=-i \operatorname{Res} \phi_{2} \neq 0$, which implies the non-vanishing of a period, a contradiction. If $k>n$, it is easy to check with (2.3) that the metric $\mathbf{g}$ vanishes, hence $\omega=0$ is a branch point.

Suppose $k=n$ or $k<n-1$. Then, (2.1),(2.2) and (2.4) imply, up to translation

$$
\begin{gathered}
x=-\frac{r^{-T}}{2 T}\left(R e a_{k} \cos (T \theta)+I m a_{k} \sin (T \theta)\right)+o\left(r^{-T}\right), \\
y=-\frac{r^{-T}}{2 T}\left(R e a_{k} \sin (T \theta)-I m a_{k} \cos (T \theta)\right)+o\left(r^{-T}\right), \\
-C r^{k+1}<z<C r^{k+1} \quad \text { if } k \neq-1, \quad z=D \ln r+O(r) \quad \text { if } k=-1,
\end{gathered}
$$

for some constants $C, D$, where $\omega=r e^{i \theta}, T:=n-k-1$. When $k=n$, it is easy to see that the image of a small circle $\left\{\omega:|\omega|=\delta^{\prime \prime}\right\} \subset D_{\delta^{\prime}}$ winds around (suitably translated) $z$-axis once, and contracts to a point as $\delta^{\prime \prime} \rightarrow 0$, hence $p=0$ is a regular point. Using (2.3), it is easy to see when it can be umbilic. When $n-1>k$, we see $x, y \rightarrow \infty$, hence $p=0$ is an end. 
The formulae in the above proof imply that the image of an end satisfies, up to translation,

$$
\begin{gathered}
|z| \leq A\left(\sqrt{x^{2}+y^{2}}\right)^{\mu} \quad \text { if } k \neq-1, \\
z=B \ln \left(\sqrt{x^{2}+y^{2}}\right)+O\left(\left(\sqrt{x^{2}+y^{2}}\right)^{-1 /(n-k-1)}\right) \quad \text { if } k=-1,
\end{gathered}
$$

for $\sqrt{x^{2}+y^{2}} \geq R$ where $A, B, R$ are some constants and $\mu:=\frac{-k-1}{n-k-1}$. Motivated by the fact that if $n-1>k>-1$ then $-(n-1) \leq \mu<0$, if $-1>k>-n-1$ then $0<\mu<1 / 2$, if $k=-n-1$ then $1 / 2=\mu$, if $-n-1>k$ then $1 / 2<\mu<1$, we classify the ends as follows.

Definition 2.1.3. We call an end planar, catenoidal, of small amplitude, of moderate amplitude, of large amplitude, respectively if $n-1>k>-1, k=$ $-1,-1>k>-n-1, k=-n-1,-n-1>k$.

The winding number of an end given by (2.4) is $n-k-1$. It is obvious that the above characterization does not depend upon a choice of the Weierstrass data. When an end is embedded, the above definition of a planar or catenoidal end coincides with what appears in literature. The definition of an immersed planar end coincides with that of a flat end of [EK].

Let $\gamma$ be a closed curve in an end $E$, and consider $\int_{\gamma} \vec{\eta}(s) d s$ where $\vec{\eta}(s)$ is a unit vector field in a neighborhood of $\gamma$, tangent to $E$ and normal to $\gamma$, pointing toward infinity. Then, the quantity $\int_{\gamma} \vec{\eta}(s) d s$ is homology invariant $[\mathrm{KKS}],[\mathrm{HK}]$.

Theorem 2.1.4 ([HK]). When $\gamma$ is any closed curve in the homology class of the puncture $p_{i} \in \bar{M}$ defining an end $E$,

$$
\operatorname{Force}(E):=\int_{\gamma} \vec{\eta}(s) d s=-2 \pi \operatorname{Res}_{p_{i}} \Phi
$$

where $\Phi$ is as in (2.2).

In particular we have $\operatorname{Res}_{p_{i}} \phi_{1}, \operatorname{Res}_{p_{i}} \phi_{2}, \operatorname{Res}_{p_{i}} \phi_{3} \in \mathbb{R}$, which is equivalent to the vanishing of the period of $E$.

Lemma 2.1.5. Suppose an end $E$ is given by (2.4). Then, we get the following table, where the vertical direction is the direction of the limit normal of an end. 


\begin{tabular}{|c|c|c|c|c|}
\hline $\begin{array}{c}\text { type } \\
\text { of end }\end{array}$ & $\begin{array}{c}\mu \\
\left(=\frac{-k-1}{n-k-1}\right)\end{array}$ & $\begin{array}{c}\text { relation of } \\
n \text { and } k\end{array}$ & $\begin{array}{c}\text { nonzero } \\
\text { horizontal force }\end{array}$ & $\begin{array}{c}\text { nonzero } \\
\text { vertical force }\end{array}$ \\
\hline planar & $-(n-1) \leq \mu<0$ & $n-1>k>-1$ & impossible & impossible \\
\hline catenoidal & $\mu=0$ & $k=-1$ & impossible & necessary \\
\hline $\begin{array}{c}\text { of small } \\
\text { amplitude }\end{array}$ & $0<\mu<\frac{1}{2}$ & $-1>k>-n-1$ & impossible & possible \\
\hline $\begin{array}{c}\text { of moderate } \\
\text { amplitude }\end{array}$ & $\mu=\frac{1}{2}$ & $k=-n-1$ & necessary & possible \\
\hline $\begin{array}{c}\text { of large } \\
\text { amplitude }\end{array}$ & $\frac{1}{2}<\mu<1$ & $-n-1>k$ & possible & possible \\
\hline
\end{tabular}

Proof. When $E$ is planar, catenoidal, or of small amplitude, we have $R e s_{0} g d h=0$. So,

$$
\operatorname{Res}_{0} \phi_{1}=\frac{1}{2} \operatorname{Res}_{0} g^{-1} d h=-i \operatorname{Re} s_{0} \phi_{2} .
$$

Since $\operatorname{Res}_{0} \phi_{1}, \operatorname{Res}_{0} \phi_{2} \in \mathbb{R}$, we conclude $\operatorname{Res}_{0} \phi_{1}=\operatorname{Res}_{0} \phi_{2}=0$, hence the horizontal components of the force vanish. It is easy to check for vertical components when $E$ is planar or catenoidal.

Suppose $E$ is an end of moderate amplitude. If horizontal components of the force vanish, then $\operatorname{Res}_{0} g^{-1} d h-R e s_{0} g d h=0=\operatorname{Res}_{0} g^{-1} d h+R e s_{0} g d h$ which implies $\operatorname{Res}_{0} g d h=a_{k}=0$, a contradiction.

All other conclusions follow from appropriate examples stated after the next corollary.

Corollary 2.1.6. If there is only one end in a complete minimal surface of nonzero finite total curvature, then it is an end of large or small amplitude.

Proof. From the balancing formula [S2][KKS] and the above table, we conclude that the end is planar or of small or large amplitude. It cannot be planar because of the harmonicity of coordinate functions and the maximum principle, by similar arguments to [JM, corollary 2].

The end of the Enneper's surface is of large amplitude. So are those of higher order Enneper's surfaces [HK]. It would be interesting to have a minimal surface with only one end which is of small amplitude.

Now, we present some examples of various ends and how to perturb them to change their forces. 
Example (i). End of large amplitude. Consider $g=\omega^{n}, d h=\omega^{-n-2}+$ $d h_{F}$ on $\mathbb{C} \cup\{\infty\}$ with $n \geq 1$ where

$$
\begin{aligned}
d h_{F}:= & \frac{\alpha}{-2 \pi}\left(-\omega^{-n-1}+\omega^{n-1}\right) d \omega \\
& +\frac{\beta}{-2 \pi}(-i)\left(\omega^{-n-1}+\omega^{n-1}\right) d \omega+\frac{\gamma}{-2 \pi} \omega^{-1} d w
\end{aligned}
$$

with $n \geq 1, \alpha, \beta, \gamma \in \mathbb{R}$. If $\alpha^{2}+\beta^{2}+\gamma^{2} \neq 0$, they give us Enneper's surface for $n=1$ or higher order Enneper's surface for $n>1$, with the only end at $\omega=0$, which is of large amplitude with vanishing force. Recall that $\omega=\infty$ is a regular point for Enneper's surface, but a branch point for higher order Enneper's' surface. If $\alpha^{2}+\beta^{2}+\gamma^{2} \neq 0$, the Weierstrass data give a branched immersion with "two" ends: $\omega=0$ is still an end of large amplitude with force $(\alpha, \beta, \gamma) ; \omega=\infty$ becomes an end of moderate amplitude if $\alpha^{2}+\beta^{2} \neq 0$, or a catenoidal end if $\alpha^{2}+\beta^{2}=0$; the branch points are uniformly away from $\omega=0$ if $\alpha^{2}+\beta^{2}+\gamma^{2}$ is small enough.

Example (ii). End of moderate amplitude. The Weierstrass data $g=\omega^{n}, d h=\left(\omega^{-n-1}-\omega^{n-1}\right) d \omega$ on $\mathbb{C} \cup\{\infty\}$ with $n \geq 1$ give us a branched immersion with two ends. Both $\omega=0$ and $\omega=\infty$ are ends of moderate amplitude with horizontal forces $(2 \pi, 0,0),(-2 \pi, 0,0)$, respectively.

Consider, on $\mathbb{C} \cup\{\infty\}$, the Weierstrass data $g=\omega^{n}$ and $d h=d h_{F}$ with $\alpha^{2}+\beta^{2}+\gamma^{2} \neq 0$ where $d h_{F}$ is as in example (i). If $\alpha^{2}+\beta^{2} \neq 0$, they give us a branched immersion with two ends. $\omega=0$ and $\infty$ are ends of moderate amplitude with forces $(\alpha, \beta, \gamma),(-\alpha,-\beta,-\gamma)$, respectively. If $\alpha^{2}+\beta^{2}=0$, they give us an $\mathrm{n}$-sheeted catenoid.

Example (iii). End of small amplitude. The Weierstrass data $g=\omega^{n}$, $d h=\left(\omega^{k}+\frac{\gamma}{-2 \pi} \omega^{-1}\right) d \omega$ on $\mathbb{C} \cup\{\infty\}$ with $n \geq 1,-1>k>-n-1, \gamma \in \mathbb{R}$ give us an immersion, branched if $\gamma \neq 0$, with two ends. $\omega=0$ is an end of small amplitude with force $(0,0, \gamma) \cdot \omega=\infty$ is planar if $\gamma=0$, or catenoidal if $\gamma \neq 0$. Creating horizontal forces for ends of small amplitude is not so simple as we will see in the proof of the following proposition.

Proposition 2.1.7. Let $E$ be an immersed end with no branch point which is catenoidal or of any amplitude, and $c>0$ be a small constant. Then for any $\vec{\xi}:=\left(\xi_{1}, \xi_{2}, \xi_{3}\right) \in \mathbb{R}^{3}$ with $\sqrt{\xi_{1}^{2}+\xi_{2}^{2}+\xi_{3}^{2}} \leq c$, we can find an immersed end $E_{\vec{\xi}}$ with no branch point such that

$$
\text { 1. } \operatorname{Force}\left(E_{\vec{\xi}}\right)-\operatorname{Force}(E)=\vec{\xi} \text {, }
$$


2. $E_{\vec{\xi}}$ depends smoothly on $\vec{\xi}$.

Proof. Without loss of generality, we may assume that $E$ is the image of $\vec{X}: D_{\delta^{\prime}} \backslash\{0\} \rightarrow \mathbb{E}^{3}$ with the Weierstrass as in (2.4). First, we note that the existence of a root of $d h=0$ in $D_{\delta^{\prime}} \backslash\{0\}$ would imply the existence of a branch point, hence it has no root.

Case (i). $E$ is an end of large or moderate amplitude. Define

$$
\tilde{g}:=g, \quad \tilde{d h}:=d h+d h_{F}=\left(1+\frac{d h_{F}}{d h}\right) d h
$$

where $d h_{F}$ is as in the example (i) with $\vec{\xi}=(\alpha, \beta, \gamma)$. Note that $1+\frac{d h_{F}}{d h}$ is a holomorphic function in $D_{\delta^{\prime}}$ in this case. When $\alpha, \beta, \gamma$ are small enough in comparison with $\delta^{\prime}$, we can say that the equation $1+\frac{d h_{F}}{d h}=0$ has no root in $D_{\delta}^{\prime}$ because $d h=0$ has no root in $D_{\delta}^{\prime} \backslash\{0\}$. Then, $\tilde{g}, \tilde{d h}$ do not create branch points and give us an immersed end of large or moderate amplitude, respectively, whose force is different from that of $E$ by $(\alpha, \beta, \gamma)$. Note that winding numbers do not change.

Case (ii). $E$ is an end of small amplitude. In this case, $a_{n-1}=0$ in (2.4) and the force of $E$ is $\left(0,0,-2 \pi a_{-1}\right)$, both of which follow from $R e s_{0} g^{-1} d h=R e s_{0} g d h=0$. (See the proof of lemma 2.1.5.) Now if we define $\tilde{g}, \tilde{d h}$ as in (2.5) we would obtain an end with a branch point in general because $\tilde{d h}$ would have a zero in $D_{\delta^{\prime}} \backslash\{0\}$ when $\alpha^{2}+\beta^{2} \neq 0$. Note that winding numbers would change. We consider instead a perturbation given by the following formulae

$$
\tilde{g}:=g\left(1+\frac{d h_{F}}{d h}\right)\left(1+A \omega^{n-k-1}\right), \quad \tilde{d h}:=\left(1+\frac{d h_{F}}{d h}\right) d h
$$

where $A=A(d h, \alpha, \beta)$ is a constant to be defined shortly. Note that $\tilde{g}$ is still holomorphic in $D_{\delta}^{\prime}$ while the order of zero at $\omega=0$ decreases in general, and that the zeros of $\tilde{d h}$ in $D_{\delta^{\prime}} \backslash\{0\}$ coincide with zeros of $\tilde{g}$ with same orders. We easily see that

$$
\tilde{g}^{-1} \tilde{d h}=\omega^{-n}\left(1-A \omega^{n-k-1}+A^{2} \omega^{2(n-k-1)}-\cdots\right) \sum_{m=k}^{\infty} a_{m} \omega^{m} d \omega
$$

which immediately implies $\operatorname{Res}_{\omega=0} \tilde{g}^{-1} \tilde{d h}=-A a_{k}$. On the other hand,

$$
\begin{aligned}
\tilde{g} \tilde{d h} & =\left(g d h+2 g d h_{F}+g d h_{F}^{2} / d h\right)\left(1+A \omega^{n-k-1}\right) \\
& =2 g d h_{F}+g d h_{F}^{2} / d h+\text { holomorphic one-form }
\end{aligned}
$$


which implies $\operatorname{Res}_{\omega=0} \tilde{g} \tilde{d h}=(\alpha+i \beta) / \pi+(\alpha+i \beta)^{2} c_{n+k+1} /\left(4 \pi^{2}\right)$ where $c_{n+k+1}$ is given by $\omega^{k} d \omega / d h=\sum_{m=0}^{\infty} c_{m} \omega^{m}$. By defining

$$
A:=\overline{(\alpha+i \beta) / \pi+(\alpha+i \beta)^{2} c_{n+k+1} /\left(4 \pi^{2}\right)} / a_{k},
$$

we have

$$
R e s_{\omega=0} \tilde{g}^{-1} \tilde{d h}=-A a_{k}=-\overline{R e s_{\omega=0} \tilde{g} \tilde{d h}} .
$$

Then, $\tilde{g}, \tilde{d h}$ on $D_{\delta^{\prime}}$ define an immersed end $\tilde{E}$ without branch points whose force is $\left(0,0,-2 \pi a_{-1}\right)+\left(-2 \pi R e\left(A a_{k}\right), 2 \pi \operatorname{Im}\left(A a_{k}\right), \gamma\right)$. The correspondence

$$
(\alpha, \beta, \gamma) \rightarrow\left(-2 \pi R e\left(A a_{k}\right), 2 \pi \operatorname{Im}\left(A a_{k}\right), \gamma\right)
$$

is a local diffeomorphism on a neighborhood of $(0,0,0)$ to a neighborhood of $(0,0,0)$, so we get the result. Note that $\tilde{E}$ is of small amplitude if $\alpha^{2}+\beta^{2}=0$ but that $\tilde{E}$ is of large amplitude if $\alpha^{2}+\beta^{2} \neq 0$. Note that winding numbers do not change.

Case (iii). $E$ is a catenoidal end. When $E$ is a catenoidal end, it already has non-zero force. Hence we resize and rotate $E$ to generate small extra force. Note that, when $\vec{\xi}=\left(\xi_{1}, \xi_{2}, \xi_{3}\right)$, we have Weierstrass data

$$
\tilde{g}=g, \quad \tilde{d h}=\sqrt{\xi_{1}^{2}+\xi_{2}^{2}+\left(\xi-2 \pi a_{-1}\right)^{2}} d h / \sqrt{\left(2 \pi a_{-1}\right)^{2}}
$$

for $E_{\vec{\xi}}$ up to rotation.

We can perturb an immersed catenoidal end (but not embedded) by perturbing the Weierstrass data as in the case (ii) of the proof, hence keeping the direction of the end. The Winding number is preserved in this case, too. When we tried the same idea to perturb embedded catenoidal ends, we always ended up producing ends with a branch point and a different winding number.

\section{Approximately minimal surfaces.}

\subsection{Construction of a family of approximately minimal surfaces.}

Conventions and definitions. For the rest of this paper, we assume the following: $\tau_{m}$ for $1 \leq m \leq K+I$ are small independent constants except that their ratios are uniformly bounded. Let

$$
\tau:=\max \left\{\tau_{m}\right\}, \quad \ell:=\ln \frac{\delta^{2}}{\tau}
$$


where $\delta$ is a small positive constant which is independent of $\tau_{m}$ 's, except that $\tau / \delta$ is very small when compared with $\delta$. Let $\psi: \mathbb{R} \rightarrow[0,1]$ be a smooth monotone function, fixed once and for all, such that $\psi \equiv 0$ on $(-\infty, 1 / 3]$ and $\psi \equiv 1$ on $[2 / 3, \infty)$. We define $\psi[a, b]: \mathbb{R} \rightarrow[0,1]$ by

$$
\psi[a, b](x):=\psi\left(\frac{x-a}{b-a}\right),
$$

where $a>b$ is allowed as well as $a<b$. We fix a Cartesian coordinate system $(x, y, z)$ in $\mathbb{E}^{3}$, and for $\vec{\xi}=\left(\xi_{1}, \xi_{2}, \xi_{3}\right) \in \mathbb{R}^{3}, \quad|\vec{\xi}|:=\sqrt{\xi_{1}^{2}+\xi_{2}^{2}+\xi_{3}^{2}}$. A function with a compact support is not distinguished from its restriction to a subdomain containing the support, or a trivial extension to a larger domain. $\lambda$ is an arbitrary constant in $\left(0,10^{-2}\right)$.

Collection of minimal surfaces. For any integer $I \geq 0$, fix $I+1$ number of complete, immersed (without branch points), orientable minimal surfaces of nonzero finite total curvature without any exceptional bounded Jacobi fields. The Weierstrass representation theorem 2.1.1 provides immersions $\tilde{X}_{i}: M_{i}=\bar{M}_{i} \backslash\left\{p_{i, q}\right\}_{q=1}^{n_{i}} \rightarrow \mathbb{E}^{3}$ for $1 \leq i \leq I+1$. We can choose a finite cover of $\bar{M}_{i}$ by open sets in such a way that each puncture $p_{i, q}, 1 \leq q \leq n_{i}$, belongs to only one open set, say $\mathcal{N}_{i, q}$. We may assume that $\mathcal{N}_{i, q}$ 's are pairwise disjoint and

$$
\mathcal{N}_{i, q}=D_{4 \delta}:=\{\omega \in \mathbb{C}:|\omega|<4 \delta\} \quad \text { with } p_{i, q}=0 .
$$

We may also assume that the Weierstrass data $g, d h$ on $\mathcal{N}_{i, q}$ are

$$
g=\omega^{n}, \quad d h=\sum_{m=k}^{\infty} a_{m} \omega^{m} d \omega \quad \text { with } a_{k} \neq 0
$$

up to Euclidean motions. We fix these data once and for all, and we do not distinguish the domains or points in $M_{i}$ from their images by $\tilde{X}_{i}$.

Now, we fix arbitrary points $\left\{p_{j}\right\}_{j=1}^{K+2 I} \subset \cup_{i=1}^{I+1} M_{i}$ where the gluing is to occur in such a way that each $M_{i}$ has at least one $p_{j}$ for $K+1 \leq p_{j} \leq K+2 I$ and that $p_{K+i}$ and $p_{K+I+i}$ for each $1 \leq i \leq I$ belong to different $M_{i}$ 's. We may assume that none of the $p_{j}$ 's belongs to the ends $\cup_{i, q} \mathcal{N}_{i, q}$.

For each $\tilde{X}_{i}: M_{i} \rightarrow \mathbb{E}^{3}$, we introduce two kinds of perturbations for the unbalancing and repositioning whose purposes are explained in the outline of the proof. 
Unbalancing. Firstly, we perturb an end so that the force of the resulting one would be different from that of the original one by a prescribed amount. Recall that if all ends of $\tilde{X}_{i}: M_{i} \rightarrow \mathbb{E}^{3}$ are planar, there is a bounded Jacobi field which does not arise from translations [EK, Theorem B]. By our assumption of no exceptional bounded Jacobi fields, we may assume without loss of generality that the end corresponding to $p_{i, 1}$ is not planar. For each $\vec{\xi}_{i} \in \mathbb{R}^{3}$ with $\left|\vec{\xi}_{\Omega_{i}}\right| \leq \gamma_{2} \ell^{2} \tau$ where $\gamma_{2}$ is a constant to be determined in proposition 4.3.4, we have an immersion $X_{i, 1}^{\prime}: D_{4 \delta} \backslash\{0\} \rightarrow \mathbb{E}^{3}$ given by proposition 2.1.7 (with $4 \delta=\delta^{\prime}$ ) the force of whose image is different from that of the original end by $\vec{\xi}_{\Omega_{i}}$. Then we define $\bar{X}_{i}: M_{i} \rightarrow \mathbb{E}^{3}$ by

$$
\bar{X}_{i}(p):=\psi(p) \tilde{X}_{i}(p)+(1-\psi(p)) X_{i, 1}^{\prime}(p)
$$

where $\psi(p):=\psi[2 \delta, 3 \delta](|\omega(p)|)$ on $D_{4 \delta} \backslash\{0\}, \quad:=1$ on $M_{i} \backslash D_{4 \delta}$.

Repositioning. Secondly, for each $p_{j} \in N_{i}$ we change the relative position of a neighborhood of $p_{j}$ and the minimal surface minus the neighborhood by translation. Let $\left(r_{j}, \theta_{j}, z_{j}\right)$ be the cylindrical coordinates system such that the the tangent plane $\subset \mathbb{E}^{3}$ at $\bar{X}_{i}\left(p_{j}\right)$ to $\bar{X}_{i}\left(M_{i}\right)$, which we call $P_{j}$, is given by $z_{j}=0$. We may assume that $\delta$ is small enough that a neighborhood of $p_{j}$ in $M_{i}$, say $\mathcal{N}_{j}$, is mapped by $\bar{X}_{i}$ to the graph over $\left\{\left(r_{j}, \theta_{j}, 0\right) \in P_{j}: r_{j}<4 \delta\right\}$ of a function, say $f_{j}$, and that $\mathcal{N}_{i, q}$ 's and $\mathcal{N}_{j}$ 's are pairwise disjoint. Define $\psi_{j}: M_{i} \rightarrow \mathbb{R}$ by $\psi_{j}:=1$ on $M_{i} \backslash \mathcal{N}_{j}, \psi_{j}:=\psi[2 \delta, 3 \delta] \circ r_{j}$ on $\mathcal{N}_{j}$. Finally, for any $\zeta_{j} \in \mathbb{R}$ with $\left|\zeta_{j}\right| \leq \gamma_{1} \ell^{2} \tau$ where $\gamma_{1}$ is a constant to be determined in proposition 4.3.4, define $\vec{X}_{i}: M_{i} \rightarrow \mathbb{E}^{3}$ by

$$
\vec{X}_{i}(p):=\bar{X}_{i}(p)+\sum_{j} \zeta_{j} \psi_{j}(p) \vec{\nu}_{i}\left(p_{j}\right)
$$

where $\vec{\nu}_{i}$ is a unit normal vector field on $\bar{X}_{i}\left(M_{i}\right)$ and the sum is taken over all indices $j$ such that $p_{j} \in M_{i}$. Note that as $\zeta_{j}$ varies, $\vec{X}_{i}\left(M_{i} \backslash \mathcal{N}_{j}\right)$ translates along $\vec{\nu}_{i}\left(p_{j}\right)$.

We now turn to construct small catenoidal ends and necks by first removing one or two catenoidal ends from an unbalanced catenoid of a fixed neck size, and then by scaling with various scale factors $\tau_{m}$.

Unbalanced catenoid. Put a catenoid $\mathcal{C}$ of neck size 1 in $\mathbb{E}^{3}$ in such a way that its axis is the $z$-axis and its center is at the origin of $\mathbb{E}^{3}$. The force of the top end is $(0,0,2 \pi)$. Choose an arbitrary $\vec{\xi}_{\mathcal{C}_{m}} \in \mathbb{R}^{3}$ with $\left|\vec{\xi}_{\mathcal{C}_{m}}\right| \leq$ $\gamma_{2} \ell^{2} \tau^{(3-2 \lambda) / 2}$. Let $\overline{\mathcal{C}}$ be another catenoid which still centered at the origin and whose top end now possesses a force of $(0,0,2 \pi)+\tau_{m}^{-1} \vec{\xi}_{\mathcal{C}_{m}}$. In fact $\overline{\mathcal{C}}$ is 
obtained from $\mathcal{C}$ rotation and homothetic expansion by small amounts. Consider the cylinder $\left\{(x, y, z): x^{2}+y^{2}=1 / 4,-1<z<1\right\}$. Both $\mathcal{C}$ and $\overline{\mathcal{C}}$ will be graphs over the cylinder given by $r=\tilde{r}(\theta, z)$ for $\mathcal{C}, \quad r=\bar{r}(\theta, z)$ for $\overline{\mathcal{C}}$, respectively, where $(r, \theta, z)$ is the cylindrical coordinate system. Let

$$
\hat{r}(\theta, z):=(1-\psi[-1,1] \circ z) \tilde{r}(\theta, z)+(\psi[-1,1] \circ z) \bar{r}(\theta, z)
$$

on $\{(\theta, z):-1<z<1\}$, and define an unbalanced catenoid $\tilde{\mathcal{C}}$ by $\tilde{\mathcal{C}}:=\mathcal{C}$ if $z<-1,:=$ the image of $(\theta, z) \mapsto \hat{r}(\theta, z)$ if $-1 \leq z \leq 1,:=\overline{\mathcal{C}}$ if $1<z$.

Small catenoidal ends and necks. Choose $\tau_{m}$ for $1 \leq m \leq K$. Cut off the bottom end of $\tilde{\mathcal{C}}$ by a plane in $\{z<0\}$ which is perpendicular to the $z$-axis and which intersects $\tilde{\mathcal{C}}$ in a circle of radius $2 \tau_{m}^{-1 / 2}$, and scale the $\tilde{\mathcal{C}}$ minus the bottom end by $\tau_{m}$, and call them $E_{m}$. That is, $E_{m}$ is the image of $\tilde{\mathcal{C}}$ by

$$
(x, y, z) \rightarrow\left(\tau_{m} x, \tau_{m} y, \tau_{m} z\right) .
$$

These are the catenoidal ends to be used in the construction. For catenoidal necks, choose $\tau_{m}$ for $K+1 \leq m \leq K+I$, and cut off the two ends of $\tilde{\mathcal{C}}$ by planes which are perpendicular to the axes of $\tilde{\mathcal{C}}$ in circles of radius $2 \tau_{m}^{-1 / 2}$, and scale the $\tilde{\mathcal{C}}$ minus the two ends by $\tau_{m}$. We call them $B_{m}$.

The configuration. Arrange the perturbed minimal surfaces $\vec{X}_{i}\left(M_{i}\right), 1 \leq$ $i \leq I+1$, the catenoidal ends $E_{m}, 1 \leq m \leq K$, and the catenoidal necks $B_{m}, K+1 \leq m \leq K+I$ as follows:

1. The boundary circle of $E_{m}$ coincides with the circle in $P_{m}$ of the same radius centered at $p_{m}$.

2. The bottom boundary circle of $B_{m}$ coincides with the circle in $P_{m}$ of the same radius centered at $p_{m}$, and the top boundary circle with the one in $P_{m+I}$ centered at $p_{m+I}$.

3. When we consider $N_{i}$ 's as vertices and $B_{m}$ 's as edges (and disregard $E_{m}$ 's), it is a connected tree.

The gluing. Now we glue them together as follows. Fix a $p_{j}$. Recall the cylindrical coordinates system $\left(r_{j}, \theta_{j}, z_{j}\right)$ where the tangent plane $P_{j}$ to $\vec{X}_{i}\left(M_{i}\right)$ at $p_{j}$ is given by $z_{j}=0$. Then, over the annulus $\tau_{j} / 4 \delta \mathrm{D}_{4 \delta}:=$ $\left\{\left(r_{j}, \theta_{j}, 0\right) \in P_{j}: \frac{\tau_{j}}{4 \delta}<r_{j}<4 \delta\right\}$, a neighborhood of $p_{j}$ in $M_{i}$ is mapped by $\vec{X}_{i}$ to the graph of a function, say $z_{j}=\bar{f}_{j}\left(r_{j}, \theta_{j}\right)$, and $E_{j}$ if $1 \leq j \leq K$ or 
$B_{j}$ if $K+1 \leq j \leq K+I$ is also the graph of a function, say $z_{j}=\tilde{f}_{j}\left(r_{j}, \theta_{j}\right)$. (We define $\tilde{\tilde{f}}_{j}:=0$ if $r_{j}>2 \sqrt{\tau_{j}}$ and may assume $\tilde{f}_{j} \geq 0$.) Define $\psi_{j}:=$ $\psi\left[\sqrt{\tau_{j}}, 2 \sqrt{\tau_{j}}\right] \circ r_{j}$ and

$$
\Phi_{j}\left(r_{j}, \theta_{j}\right):=\psi_{j} \bar{f}_{j}\left(r_{j}, \theta_{j}\right)+\left(1-\psi_{j}\right) \tilde{f}_{j}\left(r_{j}, \theta_{j}\right) \quad \text { on } \quad \tau_{j} / 4 \delta \mathrm{D}_{4 \delta} .
$$

The graph of $\Phi_{j}$ transits smoothly from $B_{m}$ or $E_{m}$ to $\vec{X}_{i}\left(M_{i}\right)$, and we glue all $\vec{X}_{i}\left(M_{i}\right), B_{m}, E_{m}$ using these transition functions to obtain a smooth, connected, orientable surface $M$ and a family of smooth complete immersions of finite total curvature $\vec{X}_{\zeta, \vec{\xi}}: M \rightarrow \mathbb{E}^{3}$ for each parameter $\left(\left(\zeta_{j}\right),\left(\vec{\xi}_{\Omega_{i}}\right),\left(\vec{\xi}_{\mathcal{C}_{m}}\right)\right)$ in $Z_{\gamma_{1}} \times \Xi_{\gamma_{2}} \times \bar{\Xi}_{\gamma_{2}}$ where

$$
\begin{gathered}
Z_{\gamma_{1}}:=\left\{\left(\zeta_{1}, \cdots, \zeta_{K+2 I}\right) \in \mathbb{R}^{K+2 I}:\left|\zeta_{j}\right| \leq \gamma_{1} \ell^{2} \tau,\right\}, \\
\Xi_{\gamma_{2}}:=\left\{\left(\vec{\xi}_{\Omega_{1}}, \cdots, \vec{\xi}_{\Omega_{I+1}}\right) \in \mathbb{R}^{3(I+1)}:\left|\vec{\xi}_{\Omega_{i}}\right| \leq \gamma_{2} \ell^{2} \tau\right\}, \\
\bar{\Xi}_{\gamma_{2}}:=\left\{\left(\vec{\xi}_{\mathcal{C}_{1}}, \cdots, \vec{\xi}_{\mathcal{C}_{K+I}}\right) \in \mathbb{R}^{3(K+I)}:\left|\vec{\xi}_{\mathcal{C}_{m}}\right| \leq \gamma_{2} \ell^{2} \tau^{(3-2 \lambda) / 2}\right\} .
\end{gathered}
$$

Remark 3.1.1. (1) It may be possible to relax the tree requirement for the graph prescribing the way the connected sums are taken, but we postpone discussing this for another occasion.

(2) Note that the positions where the gluing occurs, the sizes of the catenoidal necks and ends, and the relative sizes of $N_{i}$ 's may be prescribed, hence there are $3(K+2 I)$ continuous parameters which can be chosen freely in the construction of the approximately minimal surfaces (when there is no symmetry). There are also (discrete) choices of which sides of the minimal surfaces the catenoid is to be attached to.

Lemma 3.1.2. The immersions $\vec{X}_{\zeta, \vec{\xi}}: M \rightarrow \mathbb{R}^{3}$ depends smoothly upon $\zeta$ and $\vec{\xi}$.

Proof. follows trivially from proposition 2.1 .7 and the way the approximately minimal surfaces are constructed.

Convention. We will use subscripts ${ }_{\zeta, \vec{\xi}}$ to denote the dependency of various objects upon the parameter $\left(\left(\zeta_{j}\right),\left(\vec{\xi}_{\Omega_{i}}\right),\left(\vec{\xi}_{\mathcal{C}_{m}}\right)\right) \in Z_{\gamma_{1}} \times \Xi_{\gamma_{2}} \times \bar{\Xi}_{\gamma_{2}}$, but we try to avoid writing them if it can be clearly understood from the context. 
The smallness of the mean curvature, hence the justification for the phrase 'approximately minimal', will be shown in lemma 3.3.7.

We define $\mathcal{N}_{j} \subset M$ to be the domain which is mapped by $\vec{X}_{\zeta, \vec{\xi}}$ onto the graph of $\Phi_{j}$ over $\tau_{j} / 4 \delta \mathrm{D}_{4 \delta} \subset P_{j}$. By abusing notation, we use the same $\mathcal{N}_{i, q}$ for ends of $M_{i}$ to denote the corresponding ends of $M$. $\tilde{\mathcal{N}}_{k}$ for $1 \leq k \leq K$ is used to denote the ends of $M$ which arise from the attached catenoidal ends. Note that $\tilde{\mathcal{N}}_{k}$ may be represented up to Euclidean motion by the Weierstrass data

$$
g=\omega, \quad d h=\frac{\left|(0,0,2 \pi)+\tau_{k}^{-1} \vec{\xi}_{C_{k}}\right|}{2 \pi} \tau_{k} \frac{d \omega}{\omega} \quad \text { on } D_{4 \delta}
$$

\subsection{Conformal change of the metric.}

The smooth metrics $\mathbf{g}=\mathbf{g}_{\zeta, \vec{\xi}}$ on $\mathrm{M}$ induced by the immersions $\vec{X}_{\zeta, \vec{\xi}}: M \rightarrow$ $\mathbb{E}^{3}$ are not good for the purpose of estimating functions and tensors on $M$. In this subsection, we construct a metric conformal to $\mathrm{g}$ by gluing metrics defined locally, and then use it to estimate various quantities in the next subsection.

Firstly, we note that on the unperturbed ends $\mathcal{N}_{i, q}$ for $1 \leq i \leq I+1,2 \leq$ $q \leq n_{i}$,

$$
\mathbf{g}=\frac{\left(1+|g|^{2}\right)^{2}}{4|g|^{2}} \frac{|d h|^{2}}{|d \omega|^{2}}|\omega|^{2}\left(r^{-2} d r^{2}+d \theta^{2}\right)
$$

using (3.1) and (2.3) with $\omega=r e^{i \theta}$. Introducing $x:=\ln \frac{\delta}{r}$, we see that $r^{-2} d r^{2}+d \theta^{2}=d x^{2}+d \theta^{2}$. Define a metric $\chi_{i, q}:=d x^{2}+d \theta^{2}$ on $\mathcal{N}_{i, q}$. On the perturbed ends $\mathcal{N}_{i, 1}$, define $\chi_{i, 1}$ similarly using (3.1) and (2.5), (2.6) or (2.7), respectively, depending upon how we perturb the end $\mathcal{N}_{i, 1}$.

Secondly, on the attached catenoidal ends $\tilde{\mathcal{N}}_{k}$ for $1 \leq k \leq K$ define $x:=\ln \frac{\delta}{|\omega|}+\ln \frac{\delta^{2}}{\tau_{k}}$ and $\tilde{\chi}_{k}:=d x^{2}+d \theta^{2}$ using (3.2).

Thirdly, we note that on $\mathcal{N}_{j}$ for $1 \leq j \leq K+2 I$, where the gluing occurs, we have that

$$
\mathbf{g}=\left(1+\left(\frac{\partial \Phi_{j}}{\partial r_{j}}\right)^{2}\right) d r_{j}^{2}+2 \frac{\partial \Phi_{j}}{\partial r_{j}} \frac{\partial \Phi_{j}}{\partial \theta_{j}} d r_{j} d \theta_{j}+\left(r_{j}^{2}+\left(\frac{\partial \Phi_{j}}{\partial \theta_{j}}\right)^{2}\right) d \theta_{j}^{2}
$$

using the cylindrical coordinates system $\left(r_{j}, \theta_{j}, z_{j}\right)$ and the function $\Phi_{j}$, 
hence if $x:=\ln \frac{\delta}{r_{j}}$,

$$
\begin{aligned}
\mathbf{g}=\delta^{2} e^{-2 x} & \left(\left(1+\delta^{-2} e^{2 x}\left(\frac{\partial \Phi_{j}}{\partial x}\right)^{2}\right) d x^{2}\right. \\
+ & \left.2 \delta^{-2} e^{2 x} \frac{\partial \Phi_{j}}{\partial x} \frac{\partial \Phi_{j}}{\partial \theta_{j}} d x d \theta_{j}+\left(1+\delta^{-2} e^{2 x}\left(\frac{\partial \Phi_{j}}{\partial \theta_{j}}\right)^{2}\right) d \theta_{j}^{2}\right)
\end{aligned}
$$

Define a metric $\chi_{j}:=r_{j}^{-2} \mathbf{g}=\delta^{-2} e^{2 x} \mathrm{~g}$ on $\mathcal{N}_{j}$.

Now we define smooth cutoff functions to be used in gluing the metrics. In the following, $B_{m}^{\prime}$ or $E_{m}^{\prime}$ stands for the connected component of $M \backslash\left(\cup \mathcal{N}_{j} \cup\right.$ $\tilde{\mathcal{N}}_{k}$ ) which arises from $B_{m}$ or $E_{m}$, respectively, where the unions are taken for $1 \leq j \leq K+I, 1 \leq k \leq K$ :

1. $\psi_{i, q}:=\psi[2 \delta, \delta]$ or on $\mathcal{N}_{i, q},:=0$ on $M \backslash \mathcal{N}_{i, q}$ for $1 \leq i \leq I+1,1 \leq q \leq n_{i}$,

2. $\tilde{\psi}_{k}:=\psi[2 \delta, \delta] \circ r$ on $\tilde{\mathcal{N}}_{k},:=0$ on $M \backslash \tilde{\mathcal{N}}_{k}$ for $1 \leq k \leq K$,

3. $\psi_{j}:=\psi\left[\frac{\tau}{2 \delta}, \frac{\tau}{\delta}\right] \circ r_{j}-\psi[\delta, 2 \delta] \circ r_{j}$ on $\mathcal{N}_{j},:=0$ on $M \backslash \mathcal{N}_{j}$ for $1 \leq j \leq$ $K+2 I$,

4. $\psi_{B_{m}}:=1-\psi_{m}-\psi_{m+I}$ on $B_{m}^{\prime},:=0$ on $M \backslash B_{m}^{\prime}$ for $K+1 \leq m \leq K+I$, and $\psi_{E_{m}}:=1-\psi_{m}-\tilde{\psi}_{m}$ on $E_{m}^{\prime},:=0$ on $M \backslash E_{m}^{\prime}$ for $1 \leq m \leq K$.

Definition 3.2.1. Define a metric $\chi=\chi_{\zeta, \vec{\xi}}$ conformal to $\mathbf{g}$ by

$$
\begin{aligned}
\chi:= & \sum_{j=1}^{K+I} \psi_{j} \chi_{j}+\sum_{i=1}^{I+1} \sum_{q=1}^{n_{i}} \psi_{i, q} \chi_{i, q}+\sum_{m=K+1}^{K+I} \psi_{B_{m}} \tau_{m}^{-2} \mathbf{g} \\
& +\sum_{m=1}^{K} \psi_{E_{m}} \tau_{m}^{-2} \mathbf{g}+\sum_{k=1}^{K} \tilde{\psi}_{k} \tilde{\chi}_{k} \\
& +\left(1-\sum_{j=1}^{K+I} \psi_{j}-\sum_{i=1}^{I+1} \sum_{q=1}^{n_{i}} \psi_{i, q}-\sum_{m=K+1}^{K+I} \psi_{B_{m}}-\sum_{m=1}^{K} \psi_{E_{m}}-\sum_{k=1}^{K} \tilde{\psi}_{k}\right) \mathbf{g}
\end{aligned}
$$

A positive function $\rho_{g}: M \rightarrow \mathbb{R}$ is defined by $\chi=\rho_{g}^{2} \mathbf{g}$.

With $\chi$ metric, each end of $M$ is isometric to $S^{1} \times[0, \infty)$, and a neighborhood of the region where the gluing occurs becomes a long cylinder of length $\sim \ln \left(\delta^{2} / \tau\right)$ which is almost flat. The remaining region with $\chi$ metric 
has finite area and uniform geometry, that is nonzero injectivity radius and bounded curvature.

Now we develop some notation which is conveniently used to denote various regions of $M$.

Definition 3.2.2. (1) Define $\tau_{j}$ for $K+I+1 \leq j \leq K+2 I$ by $\tau_{j}:=\tau_{j-I}$ and $\ell_{j}:=\ln \frac{\delta^{2}}{\tau_{j}} \quad$ for $1 \leq j \leq K+2 I$.

(2) Define a function $x: M \rightarrow \mathbb{R}$ by $x(p):=\min \left\{\ell_{j}, \max \left\{0, \ln \frac{\delta}{r_{j}(p)}\right\}\right\}$ on $\mathcal{N}_{j},:=\max \left\{0, \ln \frac{\delta}{r(p)}\right\}$ on $\mathcal{N}_{i, q},:=\max \left\{\ell_{k}, \ell_{k}+\ln \frac{\delta}{r(p)}\right\}$ on $\tilde{\mathcal{N}}_{k}$, and extend it to be continuous and constant on each component of $M \backslash\left(\cup \mathcal{N}_{j} \cup \mathcal{N}_{i, q} \cup \tilde{\mathcal{N}}_{k}\right)$ where the unions are taken for $1 \leq j \leq K+2 I, 1 \leq$ $i \leq I+1,1 \leq q_{i} \leq n_{i}$, and $1 \leq k \leq K$.

(3) $V_{j}[b, c], V_{i, q}[b, c]$ or $\tilde{V}_{k}[b, c]$ is defined to be the closure of the component of $\{p \in M: b<x(p)<c\}$ which is a subset of $\mathcal{N}_{j}, \mathcal{N}_{i, q}$ or $\tilde{\mathcal{N}}_{k}$, respectively.

(4) We define closed curves $\mathcal{C}_{j}[d]:=\left\{p \in V_{j}\left[0, \ell_{j}\right]: x(p)=d\right\}, \mathcal{C}_{i, q}[d]:=$ $\left\{p \in V_{i, q}[0, \infty]: x(p)=d\right\}, \tilde{\mathcal{C}}_{k}[d]:=\left\{p \in \tilde{V}_{k}\left[\ell_{k}, \infty\right]: x(p)=d\right\}$.

(5) Define $\mathcal{C}_{m}$ to be the closure of the component of $\{p \in M: x(p)>0\}$ which arises from $E_{m}$ for $1 \leq m \leq K$ or from $B_{m}$ for $K+1 \leq m \leq$ $K+I$, and $\mathcal{C}_{m}^{\prime}:=\left\{p \in \mathcal{C}_{m}: \ell_{m}-2<x(p)<\ell_{m}+2\right\}$. Define $\Omega_{i}$ for $1 \leq i \leq I+1$ to be the closure of the component of $M \backslash \cup_{m=1}^{K+I}\{p \in$ $\left.\mathcal{C}_{m}: x(p)=\ell_{m}\right\}$ which arises from $N_{i}$, and $\Omega_{i}^{\prime}:=\left\{p \in \Omega_{i}: x(p)<2\right\}$.

Note that $\Omega_{i}$ 's and $\mathcal{C}_{m}$ 's cover $M$ and they overlap, if they do, on $V_{j}\left[0, \ell_{j}\right]$ 's. In the language of $[\mathrm{K} 5]$, we can interpret $V_{j}\left[0, \ell_{j}\right]$ as joining pieces, $\Omega_{i} \backslash \cup_{j} V_{j}\left[0, \ell_{j}\right], \mathcal{C}_{m} \backslash \cup_{j} V_{j}\left[0, \ell_{j}\right]$ as standard pieces, and $\Omega_{i}^{\prime}, \mathcal{C}_{m}^{\prime}$ as cores of the standard pieces. $V_{i, q}[0, \infty]$ or $\tilde{V}_{k}\left[\ell_{k}, \infty\right]$ are an end of $\Omega_{i}$ or of $\mathcal{C}_{k}$,respectively.

Note that $\ell / \ell_{j}$ for any $j$ is arbitrarily close to 1 when $\tau$ is small enough. Recall that $M$ is orientable. We choose a unit normal vector field on $\vec{X}_{\zeta, \vec{\xi}}(M)$ and denote it by $\vec{\nu}_{\zeta, \vec{\xi}}$. With $\chi$ metric, the minimal surface equation (1.2), parametrized by $\left(\left(\zeta_{j}\right),\left(\vec{\xi}_{\Omega_{i}}\right),\left(\vec{\xi}_{\mathcal{C}_{m}}\right)\right) \in Z_{\gamma_{1}} \times \Xi_{\gamma_{2}} \times \bar{\Xi}_{\gamma_{2}}$, can be written as

$$
\left(\Delta_{\chi}+\rho_{g}^{-2}|\mathbf{A}|^{2}\right) \phi=-2 \rho_{g}^{-2} H_{\zeta, \vec{\xi}}-2 \rho_{g}^{-2} \tilde{Q}_{\zeta, \vec{\xi}, \phi} .
$$

Definition 3.2.3. $\mathcal{L}_{\chi}=\mathcal{L}_{\zeta, \vec{\xi}, \chi}:=\Delta_{\chi}+\rho_{g}^{-2}|\mathbf{A}|^{2}$. 


\subsection{Estimates for various geometric quantities of the approximately minimal surfaces.}

Convention. From now on, we assume the following for the indices: $1 \leq$ $i \leq I+1,1 \leq q \leq n_{i}, 1 \leq j \leq K+2 I, 1 \leq k \leq K, 1 \leq m \leq K+I$. Unless stated otherwise, all indices are assumed to run through the above range, for example with sums and unions.

Let $\Phi_{j}$ be as in subsection 3.1, that is $\Phi_{j}$ is a function defined on the annulus $\left\{\left(r_{j}, \theta_{j}, 0\right) \in P_{j}: \frac{\tau_{j}}{4 \delta}<r_{j}<4 \delta\right\}$ whose graph is a neighborhood of the region where the gluing occurs. Then, $V_{j}\left[0, \ell_{j}\right]$ is the image of the embedding

$$
\vec{X}:\left[0, \ell_{j}\right] \times \mathbb{S}^{1} \rightarrow \mathbb{E}^{3}, \quad(x, \theta) \mapsto\left(\delta e^{-x} \cos \theta, \delta e^{-x} \sin \theta, \Phi_{j}\right) .
$$

In particular, if we write $x=x^{1}, \theta=x^{2}, \mathbf{g}=g_{a b} d x^{a} d x^{b}, \mathbf{A}=A_{a b} d x^{a} d x^{b}$, then

$$
A_{a b}=\frac{\operatorname{det}\left[\begin{array}{c}
\partial_{a b} \vec{X} \\
\partial_{1} \vec{X} \\
\partial_{2} \vec{X}
\end{array}\right]}{\sqrt{\operatorname{det}\left[g_{a b}\right]}}=\frac{\left|\begin{array}{ccc}
\partial_{a b}\left(\delta e^{-x} \cos \theta\right) & \partial_{a b}\left(\delta e^{-x} \sin \theta\right) & \partial_{a b} \Phi_{j} \\
-\delta e^{-x} \cos \theta & -\delta e^{-x} \sin \theta & \partial_{x} \Phi_{j} \\
-\delta e^{-x} \sin \theta & \delta e^{-x} \cos \theta & \partial_{\theta} \Phi_{j}
\end{array}\right|}{\delta^{2} e^{-2 x} \sqrt{1+\delta^{-2} e^{2 x}\left(\partial_{x} \Phi_{j}^{2}+\partial_{\theta} \Phi_{j}^{2}\right)}}
$$

For $g_{a b}$, recall (3.4). Now we proceed to estimate $\Phi_{j}$. As in [K4], for a tensor $T$,

$$
\left\|T: C^{t, \alpha}(\Omega, \chi, q)\right\|:=\sup _{p \in \Omega} \frac{\left\|T: C^{t, \alpha}\left(\Omega \cap B_{p}, \chi\right)\right\|}{q(p)}
$$

where $q$ is a function on $\Omega \subset M$ and $B_{p}$ is the geodesic ball of radius 1 centered at $p$. We omit $q$ when $q \equiv 1$.

Lemma 3.3.1. For any $t \in \mathbb{N}$, we have

$$
\left\|\nabla \Phi_{j}: C^{t}\left(V_{j}\left[0, \ell_{j}\right], d x^{2}+d \theta^{2}, q\right)\right\| \leq C(t) \delta^{2}
$$

where $q:=e^{-2 x}$ in $V_{j}\left[0, \ell_{j} / 2\right]$ and $:=e^{-\ell_{j}}$ in $V_{j}\left[\ell_{j} / 2, \ell_{j}\right]$.

Proof. Recall that $\Phi_{j}:=\psi_{j} \bar{f}_{j}+\left(1-\psi_{j}\right) \tilde{f}_{j}$ where $\psi_{j}$ is a smooth cutoff function which transits from 1 on $2 \sqrt{\tau_{j}} \leq r_{j} \leq \delta$ to 0 on $\tau_{j} / \delta \leq r_{j} \leq \sqrt{\tau_{j}}$. Since $\tilde{f}_{j}$ is analytic and $\left.\tilde{f}_{j}\right|_{r=0}=\left.D \tilde{f}_{j}\right|_{r=0}=0$, we have

$$
\tilde{f}_{j}=O\left(r_{j}^{2}\right), \quad D \tilde{f}_{j}=O\left(r_{j}\right), \quad D^{m} \tilde{f}_{j}=O(1) \quad \text { for } \sqrt{\tau} \leq r_{j} \leq \delta \quad \text { for } m \geq 2,
$$


where $D$ in this proof means $\partial_{r_{j}}$ or $r_{j}^{-1} \partial_{\theta_{j}}$. Note that

$$
\left|\tilde{f}_{j}\right| \leq C \tau_{j} \quad \text { on } \sqrt{\tau_{j}} \leq r_{j} \leq 2 \sqrt{\tau_{j}} .
$$

On the other hand, $\bar{f}_{j}$ for $\tau_{j} / \delta \leq r_{j} \leq 2 \sqrt{\tau_{j}}$ can be written as

$$
\begin{aligned}
\bar{f}_{j}\left(r_{j}, \theta_{j}\right) & =\tau_{j} \int_{r_{j} / \tau_{j}}^{2 \sqrt{\tau_{j}} / \tau_{j}} \frac{d t}{\sqrt{t^{2}-1}} \quad \text { for } 1 \leq j \leq K+I, \\
& =\tau_{j}^{\prime} \int_{r_{j} / \tau_{j}^{\prime}}^{2 \sqrt{\tau_{j}^{\prime}} / \tau_{j}^{\prime}} \frac{d t}{\sqrt{t^{2}-1}} \text { for } K+I+1 \leq j \leq K+2 I,
\end{aligned}
$$

where $\tau_{j}^{\prime}:=\tau_{j}\left|(0,0,-2 \pi)+\tau_{j}^{-1} \vec{\xi}_{C_{j-I}}\right| / 2 \pi$. (See (3.2).) Then it is easy to see

$$
\begin{gathered}
\left|\bar{f}_{j}\right| \leq C \tau_{j} \quad \text { on } \sqrt{\tau_{j}} \leq r_{j} \leq 2 \sqrt{\tau_{j}} \\
\left|D^{m} \bar{f}_{j}\right| \leq C \tau_{j} r_{j}^{-m} \quad \text { on } \tau_{j} / \delta \leq r_{j} \leq 2 \sqrt{\tau_{j}} \text { for } m \geq 1 .
\end{gathered}
$$

Now the result follows from the above estimates, the trivial estimates for $\psi_{j}$, and the relation $r_{j}=\delta e^{-x}$.

As in $[\mathrm{K} 1,4]$, two metrics $\chi_{1}$ and $\chi_{2}$ are called $t$-equivalent on a domain $\Omega$ by a constant $\mathrm{C}$ iff for $(i, j)=(1,2)$ or $(2,1)$, we have

$$
\left\|\chi_{i}: C^{t}\left(\Omega, \chi_{j}\right)\right\| \leq C, \quad\left\|\chi_{i}^{-1}: C^{t}\left(\Omega, \chi_{j}\right)\right\| \leq C .
$$

If $\chi_{1}, \chi_{2}$ are t-equivalent by $C_{1}$, then

$$
\left\|\cdot: C^{t}\left(\Omega, \chi_{1}\right)\right\| \leq C\left(t, C_{1}\right)\left\|\cdot: C^{t}\left(\Omega, \chi_{2}\right)\right\| .
$$

Lemma 3.3.2. On $\Omega_{i}^{\prime}$, $\mathbf{g}$ and $\chi$ are t-equivalent by $C(t, \delta)$. On $C_{m}^{\prime}, \tau_{m}^{-2} \mathbf{g}$ and $\chi$ are t-equivalent by $C(t, \delta)$. On $V_{j}\left[\frac{\ell_{j}}{2}-1, \frac{\ell_{j}}{2}\right], \tau_{j}^{-1} \mathbf{g}$ and $\chi$ are $t$ equivalent by $C(t)$. On $V_{j}\left[0, \ell_{j}\right], d x^{2}+d \theta^{2}$ and $\chi$ are t-equivalent by $C(t)$, and

$$
\left\|\chi-\left(d x^{2}+d \theta^{2}\right): C^{t}\left(V_{j}\left[0, \ell_{j}\right], d x^{2}+d \theta^{2}, q\right)\right\| \leq C(t) \delta^{2},
$$

where $q=e^{-2 x} \quad$ in $V_{j}\left[0, \ell_{j} / 2\right]$ and $=e^{2\left(x-\ell_{j}\right)}$ in $V_{j}\left[\ell_{j} / 2, \ell_{j}\right]$.

Proof. The equivalences of metrics on $\Omega_{i}^{\prime}$ or on $\mathcal{C}_{m}^{\prime}$ follow easily from definitions. The rest of the assertion follows from definitions, formula (3.4), and lemma 3.3.1.

In the following lemmas, $n \geq 1, k$ are integers assigned to each $V_{i, q}[0, \infty]$ by (3.1). Note that for $q=1$ they are related to the unperturbed Weierstrass data, and that $n-k-1 \geq 1$ by proposition 2.1 .2 . 
Lemma 3.3.3. When $q>1$, or $q=1$ and $V_{i, 1}[0, \infty]$ was originally (i.e. when $\left.\vec{\xi}_{i}=\overrightarrow{0}\right)$ an end of large or moderate amplitude or a catenoidal end, we have

$$
\left\|\mathbf{A}: C^{t}\left(V_{i, q}[0, \infty], \chi, e^{-(k+1) x}\right)\right\| \leq C(t) \delta^{k+1} .
$$

Note the difference of decay rates depending upon the type of ends. When $V_{i, 1}[0, \infty]$ was originally an end of small amplitude,

$$
\left\|\mathbf{A}: C^{t}\left(V_{i, 1}[0, \infty], \chi, e^{n x}\right)\right\| \leq C(t) \delta^{-n} .
$$

Finally we have

$$
\begin{gathered}
\left\|\mathbf{A}: C^{t}\left(V_{j}\left[0, \ell_{j}\right], \chi, q\right)\right\| \leq C(t) \delta^{2} \quad \text { for } q \text { as in lemma } 3.3 .1 \\
\left\|\mathbf{A}: C^{t}\left(\Omega_{i}^{\prime}, \chi\right)\right\| \leq C(t, \delta), \quad\left\|\mathbf{A}: C^{t}\left(C_{m}^{\prime}, \chi\right)\right\| \leq C(t, \delta) \tau_{m} \\
\left\|\mathbf{A}: C^{t}\left(\tilde{V}_{k}\left[\ell_{k}, \infty\right], \chi\right)\right\| \leq C(t, \delta) \tau_{k}
\end{gathered}
$$

Proof. For the first inequality with $q>1$, we see from (2.3),(3.1) that

$$
\begin{aligned}
\mathbf{A} & =\operatorname{Re}\left\{f(\omega) \omega^{k-1} d \omega^{2}\right\} \\
& =\operatorname{Re}\left\{f(\omega) \omega^{k+1}\right\}\left(d x^{2}-d \theta^{2}\right)+2 \operatorname{Im}\left\{f(\omega) \omega^{k+1}\right\} d x d \theta .
\end{aligned}
$$

for some holomorphic $f$ with $f(0) \neq 0$. From the holomorphicity of $f$, we have $\| \operatorname{Ref}($ or Imf $): C^{t}\left(D_{\delta},|d \omega|^{2}\right) \| \leq C(t)$ which in turn implies

$$
\| \operatorname{Ref}(\text { or } \operatorname{Imf}): C^{t}\left(V_{i, q}[0, \infty], \chi\right) \| \leq C(t)
$$

The first inequality with $q>1$ follows. The case for $q=1$ when $V_{i, 1}[0, \infty]$ was originally an end of large or moderate amplitude follows similarly, with perturbed Weierstrass data (2.5). When $V_{i, 1}[0, \infty]$ was originally a catenoidal end, we may consider only the effect of the scaling and get the result from (2.7).

When $V_{i, 1}[0, \infty]$ was originally an end of small amplitude, we have

$$
\mathbf{A}=\operatorname{Re}\left\{\frac{d \tilde{g}}{d \omega} f_{1}(\omega) \omega^{-n+k} d \omega^{2}\right\}
$$

from (2.6), where $f_{1}(\omega):=\left(1+A \omega^{n-k-1}\right)^{-1} \omega^{-k} d h / d \omega$. When $f_{2}:=$ $\left(-2 \pi \omega^{-k} d h / d \omega\right)^{-1}, f_{3}:=1+A \omega^{n-k-1}$, we have

$$
\tilde{g}=(-\alpha-i \beta) f_{2} f_{3} \omega^{-k-1}+f_{3} \omega^{n}+\gamma f_{2} f_{3} \omega^{n-k-1}+(\alpha-i \beta) f_{2} f_{3} \omega^{2 n-k-1}
$$


from which we compute $\frac{d \tilde{g}}{d \omega}$ to obtain

$$
\begin{aligned}
\mathbf{A}=\operatorname{Re}\left\{f_{4} \omega^{k-1} d \omega^{2}\right\}+\operatorname{Re}\{ & \left.(-\alpha-i \beta) f_{5} \omega^{-n-2} d \omega^{2}\right\} \\
& +\operatorname{Re}\left\{\gamma f_{6} d \omega^{2}\right\}+\operatorname{Re}\left\{(\alpha-i \beta) f_{7} \omega^{n-2} d \omega^{2}\right\}
\end{aligned}
$$

for some holomorphic functions $f_{4}, \cdots, f_{7}$ with $f_{i}(0) \neq 0$. Arguing as in the case for $q>1$, we obtain

$$
\left\|\mathbf{A}: C^{t}\left(V_{i, 1}[x, x+1], \chi\right)\right\| \leq C(t)\left(1+\left|\vec{\xi}_{i}\right| \delta^{-(n+k+1)} e^{(n+k+1) x}\right) \delta^{k+1} e^{-(k+1) x}
$$

which implies the result.

On $V_{j}\left[0, \ell_{j}\right]$, the result follows from (3.4), (3.7), and lemmas 3.3.1 and 3.3.2.

On $\Omega_{i}^{\prime}$, we use (2.3),(2.4) with $n=k$ to estimate $\mathbf{A}$ locally with $|d \omega|^{2}$ metric, and appeal to the equivalence of $|d \omega|^{2}$ and $\mathbf{g}$ on $D_{\delta}$, the compactness of this region with respect to the $\mathbf{g}$ metric, and finally the equivalence of $\mathbf{g}$ and $\chi$ in this region.

On $C_{m}^{\prime}$, the scaled picture by the order of $\tau_{m}^{-1}$ and the equivalence of $\tau_{m}^{-2} \mathbf{g}$ and $\chi$ give the result.

On $\tilde{V}_{k}\left[\ell_{k}, \infty\right]$, the result follows from (3.2) and (2.3).

\section{Lemma 3.3.4.}

$$
\begin{gathered}
\left\|\rho_{g}^{\mp 1}: C^{t}\left(V_{i, q}[0, \infty], \chi, e^{ \pm(n-k-1) x}\right)\right\| \leq C(t) \delta^{\mp(n-k-1)}, \\
\left\|\rho_{g}^{\mp 1}: C^{t}\left(V_{j}\left[0, \ell_{j}\right], \chi, e^{\mp x}\right)\right\| \leq C(t) \delta^{ \pm 1}, \\
\left\|\rho_{g}^{\mp 1}: C^{t}\left(C_{m}^{\prime}, \chi\right)\right\| \leq C(t, \delta) \tau_{m}^{ \pm 1}, \\
\left\|\rho_{g}^{\mp 1}: C^{t}\left(\Omega_{i}^{\prime}, \chi\right)\right\| \leq C(t, \delta), \\
\left\|\rho_{g}^{\mp 1}: C^{t}\left(\tilde{V}_{k}\left[\ell_{k}, \infty\right], \chi, e^{ \pm\left(x-\ell_{k}\right)}\right)\right\| \leq C(t)\left(\delta / \tau_{k}\right)^{\mp .}
\end{gathered}
$$

Proof. On $V_{i, q}[0, \infty]$ with $q>1$, we have from (3.1), (3.3)

$$
\rho_{g}^{-1}=\frac{1+|g|^{2}}{4|g|} \frac{|d h|}{|d \omega|}|\omega|=\frac{1+|\omega|^{2 n}}{4|\omega|^{n}}\left|\sum_{m=k}^{\infty} a_{m} \omega^{m}\right||\omega|:=F|\omega|^{-(n-k-1)} .
$$

$F$ is smooth in $D_{\delta}$, which implies $\left\|F: C^{t}\left(D_{\delta},|d \omega|^{2}\right)\right\| \leq C(t)$. Therefore $\left\|F: C^{t}\left(V_{i, q}[0, \infty], \chi\right)\right\| \leq C(t)$, and we get the result for $\rho_{g}^{-1}$. Since $\left.F\right|_{\omega=0} \neq$ $0, \frac{1}{F}$ is also smooth in $D_{\delta}$ by choosing smaller $\delta$ if necessary, we get the result for $\rho_{g}$. 
On $V_{i, q}[0, \infty]$ with $q=1$, we work with the perturbed Weierstrass data, that is $(2.5),(2.6),(2.7)$. When $V_{i, 1}[0, \infty]$ was originally an end of large or moderate amplitude, the above calculations are still valid with the perturbed Weierstrass data (2.5). When $V_{i, 1}[0, \infty]$ was originally an end of small amplitude, we observe from $(2.6),(3.3)$ that

$$
\begin{aligned}
\rho_{g}^{-1} & =\frac{1+\left|g+g \frac{d h_{F}}{d h}\right|^{2}\left|1+A \omega^{n-k-1}\right|^{2}}{4\left|1+A \omega^{n-k-1}\right|}\left|\frac{\omega^{-k} d h}{d \omega}\right||\omega|^{-(n-k-1)} \\
& :=F|\omega|^{-(n-k-1)} .
\end{aligned}
$$

We easily see that $F$ is a smooth function on $D_{\delta}$ with $\left.F\right|_{\omega=0} \neq 0$, hence arguing as in the case for $q>1$, we get the result. The result for the case where $V_{i, 1}[0, \infty]$ was originally a catenoidal end follows from (2.7).

On $V_{j}\left[0, \ell_{j}\right]$, we have $\rho_{g}^{\mp 1}=r_{i}^{ \pm 1}=\delta^{ \pm 1} e^{\mp x}$ which with the equivalence of $\chi$ and $d x^{2}+d \theta^{2}$ implies the result. On $\tilde{V}_{k}\left[\ell_{k}, \infty\right]$, it follows from (3.2). On the rest of $M$, the results follow easily from definitions.

The following corollary with lemma 3.3.2 tells us that the linearized operator $\mathcal{L}_{\chi}$ is not different much from the flat Laplacian on the joining pieces and ends.

Corollary 3.3.5. When $q>1$, or $q=1$ and $V_{i, 1}[0, \infty]$ was originally an end of large or moderate amplitude or a catenoidal end,

$$
\left\|\rho_{g}^{-2}|\mathbf{A}|^{2}: C^{t}\left(V_{i, q}[0, \infty], \chi, e^{-2 n x}\right)\right\| \leq C(t) \delta^{2 n} .
$$

When $q=1$ and $V_{i, 1}[0, \infty]$ was originally an end of small amplitude, (recall that $k+1<0$ in this case)

$$
\left\|\rho_{g}^{-2}|\mathbf{A}|^{2}: C^{t}\left(V_{i, 1}[0, \infty], \chi, e^{2(k+1) x}\right)\right\| \leq C(t) \delta^{-2(k+1)} .
$$

When $q:=e^{-2 x}$ in $V_{j}\left[0, \frac{\ell_{j}}{2}\right],:=e^{-2\left(\ell_{j}-x\right)}$ in $V_{j}\left[\frac{\ell_{j}}{2}, \ell_{j}\right]$,

$$
\left\|\rho_{g}^{-2}|\mathbf{A}|^{2}: C^{t}\left(V_{j}\left[0, \ell_{j}\right], \chi, q\right)\right\| \leq C(t) \delta^{2} .
$$

Finally

$$
\begin{aligned}
\left\|\rho_{g}^{-2}|\mathbf{A}|^{2}: C^{t}\left(\cup_{i=1}^{I+1} \Omega_{i}^{\prime} \bigcup \cup_{m=1}^{I+K} C_{m}^{\prime}, \chi\right)\right\| \leq C(t, \delta), \\
\left\|\rho_{g}^{-2}|\mathbf{A}|^{2}: C^{t}\left(\tilde{V}_{k}\left[\ell_{k}, \infty\right], \chi, e^{-2\left(x-\ell_{k}\right)}\right)\right\| \leq C(t) \delta^{2} .
\end{aligned}
$$


Proof. follows from the previous two lemmas, since $\rho_{g}^{-2}|\mathbf{A}|^{2}=\rho_{g}^{2}|\mathbf{A}|_{\chi}^{2}$ where $|\cdot|_{\chi}$ is the length with respect to the $\chi$ metric.

Definition 3.3.6. The mean curvature function $H_{\zeta, \vec{\xi}}$ related to $\vec{X}_{\zeta, \vec{\xi}}: M \rightarrow$ $\mathbb{E}^{3}$ is decomposed as follows:

$$
H_{\zeta, \vec{\xi}}=\sum_{j=1}^{K+2 I}\left(h_{\tau, j}+h_{\zeta, j}\right)+\sum_{i=1}^{I+1} h_{\vec{\xi}, \Omega_{i}}+\sum_{m=1}^{I+K} h_{\vec{\xi}, \mathcal{C}_{m}}
$$

where $\operatorname{supp}_{\tau, j} \subset V_{j}\left[\frac{\ell_{j}}{2}-1, \frac{\ell_{j}}{2}\right], \quad \operatorname{supp}_{\zeta, j} \subset \mathcal{N}_{j} \cap \cup_{i=1}^{I+1} \Omega_{i}^{\prime}, \operatorname{supp}_{\vec{\xi}, \mathcal{C}_{m}} \subset \mathcal{C}_{m}^{\prime}$, and finally $\operatorname{supp}_{\vec{\xi}, \Omega_{i}} \subset \mathcal{N}_{i, 1}$.

Lemma 3.3.7. When $\|\cdot\|$ stands for $\left\|\cdot: C^{t}(M, \chi)\right\|$, we have

$$
\begin{gathered}
\left\|\rho_{g}^{-2} h_{\tau, j}\right\| \leq C(t, \delta) \tau_{j}, \quad\left\|\rho_{g}^{-2} h_{\zeta, j}\right\| \leq C(t, \delta)\left|\zeta_{j}\right| \leq C(t, \delta) \gamma_{1} \ell_{j}^{2} \tau_{j}, \\
\left\|\rho_{g}^{-2} h_{\vec{\xi}, \Omega_{i}}\right\| \leq C(t, \delta)\left|\vec{\xi}_{\Omega_{i}}\right| \leq C(t, \delta) \gamma_{2} \ell^{2} \tau \\
\left\|\rho_{g}^{-2} h_{\vec{\xi}, \mathcal{C}_{m}}\right\| \leq C(t, \delta)\left|\vec{\xi}_{\mathcal{C}_{m}}\right| \leq C(t, \delta) \gamma_{2} \ell^{2} \tau^{(3-2 \lambda) / 2} .
\end{gathered}
$$

Proof. Recall that $H_{\zeta, \vec{\xi}}=\frac{1}{2} \sum A_{a b} g^{a b}$ in a local coordinates system. Then $\left\|h_{\tau, j}\right\| \leq C(t, \delta)$ follows from (3.4),(3.7) with lemmas 3.3.1 and 3.3.2. Trivially we have $\left\|h_{\vec{\xi}, \Omega_{i}}\right\| \leq C(t, \delta)\left|\vec{\xi}_{\Omega_{i}}\right|, \quad\left\|h_{\zeta, j}\right\| \leq C(t, \delta)\left|\zeta_{j}\right|$ from Taylor expansions of $\vec{X}_{\zeta, \vec{\xi}}: M \rightarrow \mathbb{E}^{3}$. By considering the picture scaled back by the order of $\tau_{m}^{-1}$, we see $\left\|h_{\vec{\xi}, \mathcal{C}_{m}}\right\| \leq C(t, \delta) \tau_{m}^{-2}\left|\vec{\xi}_{\mathcal{C}_{m}}\right|$. Combining these results with lemma 3.3.4, we finish the proof.

\section{Linearized equation.}

The general procedure for solving linearized equations. We will shortly find ourselves in need to solve

$$
\mathcal{L}_{\chi} \phi=E \quad \text { on } \quad M
$$

with suppE $\subset \cup_{i=1}^{I+1} \Omega_{i}^{\prime}$. Following the general approach of [K1-5], we proceed as follows: we decompose $E=\sum_{i=1}^{I+1} E_{i}$ such that supp $E_{i} \subset \Omega_{i}$. On each $\Omega_{i}$, we add a linear combination of some (vector) functions $\omega_{i}$ to $E_{i}$ so that $E_{i}+\Lambda_{i} \cdot \omega_{i}$ is orthogonal to the Dirichlet approximate kernel, hence we obtain a unique solution to

$$
\mathcal{L}_{\chi} \phi_{i}=E_{i}+\Lambda_{i} \cdot \omega_{i} \quad \text { on } \Omega_{i}, \quad \phi_{i}=0 \quad \text { on } \quad \partial \Omega_{i}
$$


Now we add a linear combination of some functions $v_{j}$ and, we obtain the exponential decay of $\phi_{i}+\sum_{j} \Theta_{j} v_{j}$ along the joining pieces $V_{j}\left[0, \ell_{j}\right] \subset \Omega_{i}$. Let $\psi_{i}: M \rightarrow \mathbb{R}$ be a smooth cutoff function which transits from 1 on $\Omega_{i}$ minus small neighborhoods of the boundary to 0 on $M \backslash \Omega_{i}$. Then, we have

$$
\begin{aligned}
\mathcal{L}_{\chi}\left(\sum_{i=1}^{I+1} \psi_{i}\left(\phi_{i}+\sum_{j} \Theta_{j} v_{j}\right)\right) \\
=E+\sum_{i=1}^{I+1} \Lambda_{i} \cdot \omega_{i}+\sum_{j}^{K+2 I} \Theta_{j} \eta_{j}+\sum_{i=1}^{I+1}\left[\mathcal{L}_{\chi}, \psi_{i}\right]\left(\phi_{i}+\sum_{j} \Theta_{j} v_{j}\right)
\end{aligned}
$$

on $M$, where $\eta_{j}:=\mathcal{L}_{\chi}\left(\psi_{i} v_{j}\right)$ and for the rest of the paper, $\left[\mathcal{L}_{\chi}, \psi\right]=\mathcal{L}_{\chi} \psi-$ $\psi \mathcal{L}_{\chi}$. Note that the last term on the right hand side of the equation is supported in $\cup_{m=1}^{I+K} \mathcal{C}_{m}^{\prime}$, which is to be rewritten as $\sum E_{m}^{\prime}$ with $\operatorname{supp} E_{m}^{\prime} \subset \mathcal{C}_{m}^{\prime}$. Add a linear combination of some (vector) functions $\bar{\omega}_{m}$ to $E_{m}^{\prime}$ so that $E_{m}^{\prime}+\bar{\Lambda}_{m} \cdot \bar{\omega}_{m}$ becomes orthogonal to the Dirichlet approximate kernel, and we get a unique solution to

$$
\mathcal{L}_{\chi} \phi_{m}^{\prime}=E_{m}^{\prime}+\bar{\Lambda}_{m} \cdot \bar{\omega}_{m} \quad \text { on } \mathcal{C}_{m}
$$

Let $\psi_{m}^{\prime}$ be a smooth cutoff function which transits smoothly from 1 on $\mathcal{C}_{m}$ minus small neighborhood of the boundary to 0 on $M \backslash \mathcal{C}_{m}$. Then

$$
\begin{aligned}
\mathcal{L}_{\chi}\left(\sum_{i=1}^{I+1} \psi_{i}\left(\phi_{i}+\Theta_{j} v_{j}\right)\right. & \left.+\sum_{m=1}^{I+K} \psi_{m}^{\prime} \phi_{m}^{\prime}\right) \\
= & E+\sum_{i=1}^{I+1} \Lambda_{i} \cdot \omega_{i}+\sum_{j=1}^{K+2 I} \Theta_{j} \eta_{j}+\sum_{m=1}^{I+K} \bar{\Lambda}_{m} \cdot \bar{\omega}_{m}+E^{\prime \prime}
\end{aligned}
$$

on $M$ with supp $E^{\prime \prime} \subset \cup_{i=1}^{I+1} \Omega_{i}^{\prime}$. Then we iterate the process to obtain $\phi$ which satisfies

$$
\mathcal{L}_{\chi} \phi=E+\sum_{i=1}^{I+1} \Lambda_{i} \cdot \omega_{i}+\sum_{i=j}^{I+1} \Theta_{j} \eta_{j}+\sum_{m=1}^{I+K} \bar{\Lambda}_{m} \cdot \bar{\omega}_{m} \quad \text { on } M .
$$

The reason for the perturbations for unbalancing and repositioning can now be explained. Consider the linearized minimal surface equation. Recall that $\sum_{j} h_{\tau, j}$ is caused by the gluing, and that 
$\sum_{i} h_{\vec{\xi}, \Omega_{i}}+\sum_{m} h_{\vec{\xi}, \mathcal{C}_{m}}$ and $\sum_{j} h_{\zeta, j}$ are artificially introduced for unbalancing and repositioning, respectively. For $\sum_{j} h_{\tau, j}$, a slight modification of the general procedure produces $\phi_{\tau}$ and some unwanted terms such that

$$
\mathcal{L}_{\chi} \phi_{\tau}=-2 \rho_{g}^{-2} \sum h_{\tau, j}+\sum \Lambda_{\tau, i} \cdot \omega_{i}+\sum \bar{\Lambda}_{\tau, m} \cdot \bar{\omega}_{m}+\sum \Theta_{\tau, j} \eta_{j} .
$$

Then we construct $\phi_{\vec{\xi}}$ following the general procedure, and $\phi_{\zeta}$ in somewhat different manner, which satisfy

$$
\begin{aligned}
\mathcal{L}_{\chi} \phi_{\zeta}= & -2 \rho_{g}^{-2} \sum h_{\zeta, i}+\sum \Lambda_{\zeta, i} \cdot \omega_{i}+\sum \bar{\Lambda}_{\zeta, m} \cdot \bar{\omega}_{m}+\sum \Theta_{\zeta, j} \eta_{j}, \\
\mathcal{L}_{\chi} \phi_{\vec{\xi}}= & -2 \rho_{g}^{-2}\left(\sum h_{\vec{\xi}, \Omega_{i}}+\sum h_{\vec{\xi}, \mathcal{C}_{m}}\right)+\sum \Lambda_{\vec{\xi}, i} \cdot \omega_{i} \\
& +\sum \bar{\Lambda}_{\vec{\xi}, m} \cdot \bar{\omega}_{m}+\sum \Theta_{\vec{\xi}, j} \eta_{j}
\end{aligned}
$$

with

$$
\Lambda_{\tau, i}-\Lambda_{\vec{\xi}, i} \sim 0 \sim \Lambda_{\zeta, i}, \quad \bar{\Lambda}_{\tau, m}-\bar{\Lambda}_{\vec{\xi}, m} \sim 0 \sim \bar{\Lambda}_{\zeta, m}, \quad \Theta_{\tau, j}-\Theta_{\zeta, j} \sim 0 \sim \Theta_{\vec{\xi}, j} .
$$

So for each parameter in $Z_{\gamma_{1}} \times \Xi_{\gamma_{2}} \times \bar{\Xi}_{\gamma_{2}}$, we have $\Phi_{\zeta, \vec{\xi}}:=\phi_{\tau}+\phi_{\zeta}+\phi_{\vec{\xi}}$ which satisfies

$$
\mathcal{L}_{\chi} \Phi_{\zeta, \vec{\xi}} \sim-2 \rho_{g}^{-2} H_{\zeta, \vec{\xi}}
$$

Our hope is that all $\sim$ are actually $=$ for some parameter $\zeta, \vec{\xi}$.

\subsection{Linearized equation on joining pieces and ends..}

In this subsection, we study the existence, uniqueness, and the decay property of solutions of linearized equations on joining pieces $V_{j}\left[0, \ell_{j}\right]$ and ends $V_{i, q}[0, \infty], \tilde{V}_{k}\left[\ell_{k}, \infty\right]$. Note that the linearized operator $\mathcal{L}_{\chi}$ on these domains is a small perturbation of the Laplacian $\Delta_{s}$ of the flat metric $s:=d x^{2}+d \theta^{2}$ due to lemma 3.3.2 and corollary 3.3.5. The situation is quite similar to [K5,Appendix A], but our metric and the 0 -th order term on $V_{j}\left[\ell_{j} / 2, \ell_{j}\right]$ show different behavior. Furthermore our strategy of obtaining local solutions on joining pieces is different from that of [K5], and requires us to analyze our situation more carefully.

Conventions. We use $\tilde{\varepsilon}$ to denote a constant which can be taken arbitrarily small by choosing smaller $\delta$ if necessary, and we fix a constant $\alpha \in(0,1)$.

Lemma 4.1.1. The smallest eigenvalue for the Dirichlet problem for $\mathcal{L}_{\chi}$ on any domain of $V_{j}\left[0, \ell_{j}\right]$ is no smaller than $C \ell_{j}^{-2}$. In particular, the Dirichlet problem has a unique solution. 
Proof. By the domain monotonicity and variational characterization of eigenvalues [C], and by lemma 3.3.2, it suffices to show, where $V:=V_{j}\left[0, \ell_{j}\right]$,

$$
\iint_{V} \phi^{2} d x d \theta \leq C \ell_{j}^{2} \iint_{V}\left(|\nabla \phi|_{s}^{2}-4 \rho_{g}^{-2}|\mathbf{A}|^{2} \phi^{2}\right) d x d \theta
$$

for any smooth function $\phi$ vanishing on $\partial V$. For this, we first decompose $\phi(x, \theta)=\phi_{\text {avg }}(x)+\phi_{o s c}(x, \theta)$ by $\phi_{\text {avg }}(x):=\frac{1}{2 \pi} \int_{0}^{2 \pi} \phi(x, \theta) d \theta$, and observe that

$$
\begin{gathered}
\iint_{V} q(x) \phi^{2}(x, \theta) d x d \theta=\iint_{V} q(x) \phi_{o s c}^{2}(x, \theta) d x d \theta+2 \pi \int_{0}^{\ell_{j}} q(x) \phi_{a v g}^{2}(x) d x, \\
\phi_{o s c}^{2}(x, \theta) \leq 2 \pi \int_{0}^{2 \pi}|\nabla \phi(x, \theta)|_{s}^{2} d \theta \quad \text { for } x \in\left[0, \ell_{j}\right], \\
\phi_{a v g}^{2}(x) \leq x \int_{0}^{x}\left(\phi_{a v g}^{\prime}(\tilde{x})\right)^{2} d \tilde{x} \leq x \iint_{V}|\nabla \phi(\tilde{x}, \theta)|_{s}^{2} d \tilde{x} d \theta \quad \text { for } x \in\left[0, \ell_{j} / 2\right], \\
\phi_{a v g}^{2}(x) \leq\left(\ell_{j}-x\right) \int_{x}^{\ell_{j}}\left(\phi_{\text {avg }}^{\prime}(\tilde{x})\right)^{2} d \tilde{x} \leq\left(\ell_{j}-x\right) \iint_{V}|\nabla \phi(\tilde{x}, \theta)|_{s}^{2} d \tilde{x} d \theta
\end{gathered}
$$

for $x \in\left[\ell_{j} / 2, \ell_{j}\right]$. The above with $q \equiv 1$ or $q$ as in corollary 3.3 .5 imply

$$
\begin{aligned}
\iint_{V} \phi^{2} d x d \theta & \leq C \ell_{j}^{2} \iint_{V}|\nabla \phi|_{s}^{2} d x d \theta, \\
\iint_{V} \rho_{g}^{-2}|\mathbf{A}|^{2} \phi^{2} d x d \theta & \leq C \tilde{\varepsilon} \iint_{V}|\nabla \phi|_{s}^{2} d x d \theta .
\end{aligned}
$$

The result follows.

Corollary 4.1.2. If $\mathcal{L}_{\chi} \phi_{1}=\mathcal{L}_{\chi} \phi_{2}$ in $\Omega \subset V_{j}\left[0, \ell_{j}\right]$ and $\phi_{1} \geq \phi_{2}$ on $\partial \Omega$, then $\phi_{1} \geq \phi_{2}$ on $\Omega$.

Proof. If $\Omega^{\prime}:=\left\{p \in \Omega: \phi_{1}(p)<\phi_{2}(p)\right\}$ is not empty, then $\mathcal{L}_{\chi}\left(\phi_{1}-\phi_{2}\right)=0$ in $\Omega^{\prime}$ and $\phi_{1}-\phi_{2}=0$ on $\partial \Omega^{\prime}$. But, then we should have $\phi_{1}-\phi_{2}=0$ in $\Omega^{\prime}$ by the previous lemma, a contradiction.

Proposition 4.1.3. Let $V:=V_{j}\left[x_{0}, x_{1}\right]$ where $0 \leq x_{0}<x_{1} \leq \ell_{j}$ with $x_{1}-x_{0}$ large enough, say $x_{1}-x_{0} \geq \ell_{j} / 4$. Or let $V:=V_{i, q}\left[x_{0}, \infty\right]$ with $x_{0}=0$, or $V:=\tilde{V}_{k}\left[x_{0}, \infty\right]$ with $x_{0}=\ell_{k}$. Let $\lambda_{0}$ be an arbitrary number in 
$(0,1)$. Then, there are bounded linear mappings

$$
\begin{gathered}
\mathcal{R}_{1}: C^{0, \alpha}\left(V, s, e^{-\left(1-\lambda_{0}\right)\left(x-x_{0}\right)}\right) \rightarrow C^{2, \alpha}\left(V, s, e^{-\left(1-\lambda_{0}\right)\left(x-x_{0}\right)}\right), \\
\mathcal{R}_{2}: C^{0, \alpha}\left(V_{j}\left[x_{0}, x_{1}\right], s, e^{-\left(1-\lambda_{0}\right)\left(x_{1}-x\right)}\right) \rightarrow C^{2, \alpha}\left(V_{j}\left[x_{0}, x_{1}\right], s, e^{-\left(1-\lambda_{0}\right)\left(x_{1}-x\right)}\right)
\end{gathered}
$$

which satisfy, if $\phi_{E}$ is the image of $E$ by $\mathcal{R}_{1}$ or $\mathcal{R}_{2}$,

1. $\mathcal{L}_{\chi} \phi_{E}=E$ in $V$.

2. For $\mathcal{R}_{1}$, we have $\phi_{E}=a_{E} \in \mathbb{R}$ on $\mathcal{C}_{j}\left[x_{0}\right], \phi_{E}=0$ on $\mathcal{C}_{j}\left[x_{1}\right]$ if $V=$ $V_{j}\left[x_{0}, x_{1}\right]$; otherwise $\phi_{E}=a_{E} \in \mathbb{R}$ on $\mathcal{C}_{j}\left[x_{0}\right]$ or on $\tilde{\mathcal{C}}_{k}\left[x_{0}\right]$.

For $\mathcal{R}_{2}$, we have $\phi_{E}=a_{E} \in \mathbb{R}$ on $\mathcal{C}_{j}\left[x_{1}\right]$ and $\phi_{E}=0$ on $\mathcal{C}_{j}\left[x_{0}\right]$.

3. For $\mathcal{R}_{1}$,

$$
\begin{aligned}
\left\|\phi_{E}: C^{2, \alpha}\left(V, s, e^{-\left(1-\lambda_{0}\right)\left(x-x_{0}\right.}\right)\right\| & \\
& \leq C\left(\lambda_{0}\right)\left\|E: C^{0, \alpha}\left(V, s, e^{-\left(1-\lambda_{0}\right)\left(x-x_{0}\right)}\right)\right\| .
\end{aligned}
$$

For $\mathcal{R}_{2}$,

$$
\begin{array}{r}
\left\|\phi_{E}: C^{2, \alpha}\left(V, s, e^{-\left(1-\lambda_{0}\right)\left(x_{1}-x\right)}\right)\right\| \\
\quad \leq C\left(\lambda_{0}\right)\left\|E: C^{0, \alpha}\left(V, s, e^{-\left(1-\lambda_{0}\right)\left(x_{1}-x\right)}\right)\right\| .
\end{array}
$$

4. For $\mathcal{R}_{1},\left|a_{E}\right| \leq C\left(\lambda_{0}\right)\left\|E: C^{0, \alpha}\left(V, s, e^{-\left(1-\lambda_{0}\right)\left(x-x_{0}\right)}\right)\right\|$.

For $\mathcal{R}_{2},\left|a_{E}\right| \leq C\left(\lambda_{0}\right)\left\|E: C^{0, \alpha}\left(V, s, e^{-\left(1-\lambda_{0}\right)\left(x_{1}-x\right)}\right)\right\|$.

Proof. By standard theory, it is easy to construct a bounded linear mapping $i_{\Delta_{s}}$

$$
\begin{gathered}
C^{0, \alpha}\left(V, s, e^{-\left(1-\lambda_{0}\right)\left(x-x_{0}\right)}\right) \rightarrow C^{2, \alpha}\left(V, s, e^{-\left(1-\lambda_{0}\right)\left(x-x_{0}\right)}\right) \quad \text { or } \\
C^{0, \alpha}\left(V_{j}\left[x_{0}, x_{1}\right], s, e^{-\left(1-\lambda_{0}\right)\left(x_{1}-x\right)}\right) \rightarrow C^{2, \alpha}\left(V_{j}\left[x_{0}, x_{1}\right], s, e^{-\left(1-\lambda_{0}\right)\left(x_{1}-x\right)}\right)
\end{gathered}
$$

which satisfies $2,3,4$, and 1 with $\Delta_{s}$ instead of $\mathcal{L}_{\chi}$. Then, $i_{\Delta_{s}}$ is the inverse of $\Delta_{s}$ which is regarded as a mapping from the range of $i_{\Delta_{s}}$ to $C^{0, \alpha}\left(V, s, e^{-\left(1-\lambda_{0}\right)\left(x-x_{0}\right)}\right)$ (or $C^{0, \alpha}\left(V_{j}\left[x_{0}, x_{1}\right], s, e^{-\left(1-\lambda_{0}\right)\left(x_{1}-x\right)}\right)$ ). Since the operator norm of $\mathcal{L}_{\chi}-\Delta_{s}$ is small (in particular $\leq \tilde{\varepsilon}$ ) by lemma 3.3.2 and corollary 3.3 .5 , we have the result. 
The map $\mathcal{R}_{2}$ will be used only in the proof of lemma 4.1.5.

In the following proposition, $\lambda$ is the constant used for the range of $\vec{\xi}_{\mathcal{C}_{m}}$. Note that $\phi$ in the next proposition shows a linear decay in general as is seen from lemma 4.1.5 and corollary 4.1.2.

Proposition 4.1.4. Let $\bar{v}_{j}$ be the solution to $\mathcal{L}_{\chi} \bar{v}_{j}=0$ in $V_{j}\left[\frac{\ell_{j}}{2}, \ell_{j}\right], \bar{v}_{j}=1$ on $C_{j}\left[\frac{\ell_{j}}{2}\right], \bar{v}_{j}=0$ on $\mathcal{C}_{j}\left[\ell_{j}\right]$. Suppose

$$
\mathcal{L}_{\chi} \phi=0 \quad \text { in } V_{j}\left[\ell_{j} / 2, \ell_{j}\right], \quad \phi=0 \text { on } \mathcal{C}_{j}\left[\ell_{j}\right]
$$

Then, there exists $a_{\phi} \in \mathbb{R}$, unique by the construction, such that

$$
\begin{aligned}
& \left\|\phi+a_{\phi} \bar{v}_{j}: C^{2, \alpha}\left(V_{j}\left[\frac{\ell_{j}}{2}, \ell_{j}\right], \chi, e^{-(1-\lambda)\left(x-\ell_{j} / 2\right)}\right)\right\|
\end{aligned}
$$

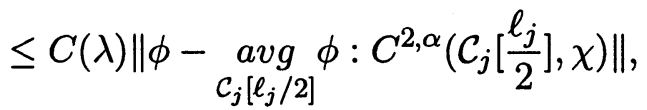

(2)

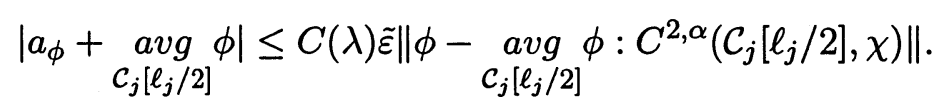

Proof. Let $\Delta_{s} \tilde{v}_{j}=0$ in $V_{j}\left[\ell_{j} / 2, \ell_{j}\right], \tilde{v}_{j}=1$ on $\mathcal{C}_{j}\left[\ell_{j} / 2\right], \tilde{v}_{j}=0$ on $\mathcal{C}_{j}\left[\ell_{j}\right]$. Define $\varphi$ by

$$
\Delta_{s} \varphi=0 \quad \text { in } V_{j}\left[\ell_{j} / 2, \ell_{j}\right], \quad \varphi=\phi \quad \text { on } \partial V_{j}\left[\ell_{j} / 2, \ell_{j}\right] .
$$

By standard theory using Fourier expansion on a circle, it is not hard to see that if $a_{\varphi}=-\operatorname{avg} \varphi$, then (1) with 0 instead of $\lambda$ is satisfied with $\varphi, a_{\varphi}, \tilde{v}_{j}, s$ instead of $\phi, a_{\phi}, \bar{v}_{j}, \chi$, and

$$
\begin{aligned}
& \left\|\mathcal{L}_{\chi}\left(\varphi+a_{\varphi} \tilde{v}_{j}\right): C^{0, \alpha}\left(V_{j}\left[\ell_{j} / 2, \ell_{j}\right], s, e^{-\left(x-\ell_{j}\right) / 2}\right)\right\| \\
& \leq C \tilde{\varepsilon} \| \varphi-\underset{\mathcal{C}_{j}\left[\ell_{j} / 2\right]}{\operatorname{avg} \varphi: C^{2, \alpha}\left(\mathcal{C}_{j}\left[\ell_{j} / 2\right], s\right) \| .}
\end{aligned}
$$


If $\varphi^{\prime}$ is the image of $-\mathcal{L}_{\chi}\left(\varphi+a_{\varphi} \tilde{v}_{j}\right)$ by $\mathcal{R}_{1}$ of the previous proposition with $\lambda_{0}=\lambda$, then

$$
\varphi+a_{\varphi} \tilde{v}_{j}+\varphi^{\prime}=\phi+\left(a_{\varphi}+\left.\varphi^{\prime}\right|_{\mathcal{C}_{j}\left[\ell_{j} / 2\right]}\right) \bar{v}_{j} \quad \text { on } V_{j}\left[\ell_{j} / 2, \ell_{j}\right]
$$

by lemma 4.1 .1 because they coincide on $\mathcal{C}_{j}\left[\ell_{j} / 2\right] \cup \mathcal{C}_{j}\left[\ell_{j}\right]$ and have the same image (i.e. 0 ) by $\mathcal{L}_{\chi}$. Define $a_{\phi}:=a_{\varphi}+\left.\varphi^{\prime}\right|_{\mathcal{C}_{j}\left[\ell_{j} / 2\right]}$. Finally we change all norms in terms of $\chi$ by appealing to lemma 3.3.2.

Lemma 4.1.5. Suppose $0 \leq t<u \leq \ell_{j}$ with $u-t$ large enough, say $u-t \geq$ $\ell_{j} / 4$, and $\phi_{l}, \phi_{r}$ satisfy

$$
\begin{gathered}
\mathcal{L}_{\chi} \phi_{l}=\mathcal{L}_{\chi} \phi_{r}=0 \quad \text { in } V_{j}[t, u] \\
\phi_{l}-1=\phi_{r}=0 \quad \text { on } \mathcal{C}_{j}[t], \quad \phi_{l}=\phi_{r}-1=0 \quad \text { on } \mathcal{C}_{j}[u]
\end{gathered}
$$

Then, on $V_{j}[t, u]$,

$$
\left|\phi_{l}-\frac{u-x}{u-t}\right| \leq \tilde{\varepsilon}, \quad\left|\phi_{r}-\frac{x-t}{u-t}\right| \leq \tilde{\varepsilon} .
$$

Proof. First consider the case where $t \leq \ell / 2 \leq u$. Let $\varphi_{l}=(u-x) /(u-t)$ and $\varphi_{r}=(x-t) /(u-t)$. Define $E_{1}^{l}:=-\mathcal{L}_{\chi} \varphi_{l} \cdot \psi[\ell / 2-1, \ell / 2] \circ x, E_{2}^{l}:=-\mathcal{L}_{\chi} \varphi_{l}-E_{1}^{l}$ and $E_{1}^{r}, E_{2}^{r}$ similarly with $\varphi_{r}$ instead of $\varphi_{l}$, then

$$
\begin{array}{r}
\left\|E_{1}^{l}\left(E_{1}^{r}\right): C^{0, \alpha}\left(V_{j}[t, u], s, e^{-2(x-t)}\right)\right\| \leq \tilde{\varepsilon}, \\
\left\|E_{2}^{l}\left(E_{2}^{r}\right): C^{0, \alpha}\left(V_{j}[t, u], s, e^{-2(u-x)}\right)\right\| \leq \tilde{\varepsilon} .
\end{array}
$$

In particular, $E_{1}^{l}, E_{1}^{r}$ are in the domain of $\mathcal{R}_{1}$ (of proposition 4.1.3), and $E_{2}^{l}, E_{2}^{r}$ are in the domain of $\mathcal{R}_{2}$ with $\lambda_{0}=1 / 2$. For $i=1,2$, let $\phi_{i}^{l}, a_{i}^{l}$ be given by proposition 4.1 .3 with $E_{i}^{l}$ and $\phi_{i}^{r}, a_{i}^{r}$ with $E_{i}^{r}$. Then we observe

$$
\begin{gathered}
\left\|\phi_{i}^{l}: C^{2, \alpha}\left(V_{j}[t, u], s, e^{-(x-t) / 2}\right)\right\|, \quad\left|a_{i}^{l}\right| \leq C \tilde{\varepsilon}, \\
\left\|\phi_{i}^{r}: C^{2, \alpha}\left(V_{j}[t, u], s, e^{-(u-x) / 2}\right)\right\|, \quad\left|a_{i}^{r}\right| \leq C \tilde{\varepsilon}, \\
\mathcal{L}_{\chi}\left(\phi_{1}^{l}+\phi_{2}^{l}+\varphi_{l}\right)=\mathcal{L}_{\chi}\left(\phi_{1}^{r}+\phi_{2}^{r}+\varphi_{r}\right)=0 \quad \text { in } V_{j}[t, u], \\
\phi_{1}^{l}+\phi_{2}^{l}+\varphi_{l}=1+a_{1}^{l} \quad \text { on } \mathcal{C}_{j}[t],=a_{2}^{l} \quad \text { on } \mathcal{C}_{j}[u], \\
\phi_{1}^{r}+\phi_{2}^{r}+\varphi_{r}=a_{1}^{r} \quad \text { on } \mathcal{C}_{j}[t],=1+a_{2}^{r} \quad \text { on } \mathcal{C}_{j}[u],
\end{gathered}
$$


so by corollary 4.1 .2 ,

$$
\begin{aligned}
\phi_{1}^{l}+\phi_{2}^{l}+\varphi_{l}=\left(a_{1}^{l}+1\right) \phi_{l}+a_{2}^{l} \phi_{r} & \text { in } V_{j}[t, u], \\
\phi_{1}^{r}+\phi_{2}^{r}+\varphi_{r}=a_{1}^{r} \phi_{l}+\left(1+a_{2}^{r}\right) \phi_{r} & \text { in } V_{j}[t, u],
\end{aligned}
$$

hence we obtain

$$
\left(\begin{array}{c}
\phi_{l} \\
\phi_{r}
\end{array}\right)=\frac{1}{\left(1+a_{1}^{l}\right)\left(1+a_{2}^{r}\right)-a_{1}^{r} a_{2}^{l}}\left(\begin{array}{cc}
1+a_{2}^{r} & -a_{2}^{l} \\
-a_{1}^{r} & 1+a_{1}^{l}
\end{array}\right)\left(\begin{array}{c}
\phi_{1}^{l}+\phi_{2}^{l}+\varphi_{l} \\
\phi_{1}^{r}+\phi_{2}^{r}+\varphi_{r}
\end{array}\right),
$$

which implies the result (with $\delta$ chosen small enough). The proofs for other cases follow similarly.

\subsection{Linearized equation with an arbitrary inhomogeneous term.}

Dirichlet problems on $\mathcal{C}_{m}$. We need to solve

$$
\mathcal{L}_{\chi} \phi=E \quad \text { in } \mathcal{C}_{m}, \quad \phi=0 \quad \text { on } \partial \mathcal{C}_{m} .
$$

Recall that $\mathcal{C}_{m}$ is obtained from a catenoid with one or two ends removed ( and extended by one or two joining pieces). A catenoid equipped with the metric $\frac{|\mathbf{A}|^{2}}{2} \mathbf{g}$ is isometric to the standard unit sphere minus two points, hence $\mathcal{C}_{m}$ equipped with the metric $\frac{|\mathbf{A}|^{2}}{2} \mathbf{g}$ can be thought of as a small perturbation of the unit standard sphere. Then [K1,Appendix B] could be used to compare low eigenvalues and corresponding eigenfunctions of $\Delta_{\mathrm{g}^{2}}+2$ on $\mathbb{S}^{2}$ and $\Delta_{\frac{|\mathbf{A}|^{2}}{2} \mathbf{g}}+2$ on $\mathcal{C}_{m}$. Note that the above equation could be rewritten as

$$
\left(\Delta_{\frac{|\mathbf{A}|^{2}}{2} \mathbf{g}}+2\right) \phi=\frac{2}{|\mathbf{A}|^{2}} \rho_{g}^{2} E \quad \text { in } \mathcal{C}_{m}, \quad \phi=0 \quad \text { on } \partial \mathcal{C}_{m}
$$

But, $|\mathbf{A}|^{2}$ may possibly vanish on $V_{j}\left[\frac{\ell_{j}}{2}-1, \frac{\ell_{j}}{2}\right] \subset \mathcal{C}_{m}$, which makes the equation meaningless. For this reason, we consider a metric on $\mathcal{C}_{m}$ which is $\frac{|\mathbf{A}|^{2}+\delta_{1}}{2} \mathbf{g}$ on $V_{j}\left[\frac{\ell_{j}}{2}-1, \frac{\ell_{j}}{2}\right]$, where $\delta_{1}$ is a positive constant which can be taken arbitrarily small.

Dirichlet problems on $\Omega_{i}$. The situation, however, becomes subtler when we consider a Dirichlet problem on $\Omega_{i}$ because of the presence of umbilic points, where $|\mathbf{A}|^{2}=0$, and ends. Recall that each end $V_{i, q}[0, \infty]$ 
is given, up to Euclidean motion, by $(2.1),(2.2)$ with the Weierstrass data. (3.1) for $q>1$ or $(2.5),(2.6),(2.7)$ if $q=1$. We have

$$
\frac{|\mathbf{A}|^{2}}{2} \mathbf{g}=\frac{4\left|g^{\prime}\right|^{2}}{\left(1+|g|^{2}\right)^{2}}|d \omega|^{2} \quad \text { on } V_{i, q}[0, \infty]=D_{\delta} \backslash\{0\},
$$

where ' means the complex differentiation regarding $g$ as a function on $D_{\delta}$, which shows that $\frac{|\mathbf{A}|^{2}}{2} \mathbf{g}$ becomes singular at $p_{i, q}=0$ iff $n \geq 2$. Note that this phenomenon does not occur with a catenoid since it has $n=1$. So we define a small perturbation of the metric $\frac{|\mathbf{A}|^{2}}{2} \mathbf{g}$ on an end by the following formula

$$
h_{i, q}:=\frac{4\left|g^{\prime}\right|^{2}+\delta_{1}}{\left(1+|g|^{2}\right)^{2}}|d \omega|^{2} \quad \text { on } V_{i, q}[0, \infty]=D_{\delta} \backslash\{0\}
$$

which can be smoothly extended on the entire $D_{\delta}$.

Motivated by the above discussion, we now define a (nonsingular) metric on $M$ by slightly perturbing the pull-back of the metric of the sphere.

Definition 4.2.1. Let $\delta_{1}$ be a constant which is small and independent of $\delta, \tau$. Let $\psi_{i, q}$ be a smooth cutoff function on $\bar{M}:=M \cup\{$ punctures $\}$ defined by $:=\psi[1,2] \circ x$ on $V_{i, q}[0, \infty],:=1$ on $\{$ puncture $\}$, and $:=0$ elsewhere on $\bar{M}$, and let $\psi$ be a smooth cutoff function which is $\psi\left[\ell_{j}-1, \ell_{j}\right] \circ x$ on $V_{j}\left[0, \ell_{j}\right]$ and is constant on each component of $M \backslash \cup V_{j}\left[0, \ell_{j}\right]$. Define a smooth metric $h$ on $\bar{M}$ by

$$
h:=\psi \frac{|\mathbf{A}|^{2}}{2} \mathbf{g}+\sum_{i, q} \psi_{i, q} h_{i, q}+\left(1-\psi-\sum_{i, q} \psi_{i, q}\right) \frac{|\mathbf{A}|^{2}+\delta_{1}}{2} \mathbf{g} .
$$

$\tilde{\rho}_{g}: \bar{M} \rightarrow \mathbb{R}$ is defined by $h=\tilde{\rho}_{g}^{2} \mathbf{g}$ on $M, \tilde{\rho}_{g}^{2}:=0$ at punctures.

Before giving the following definition, we note

$$
\tilde{\rho}_{g}^{-2}|\mathbf{A}|^{2}=\frac{8\left|g^{\prime}\right|^{2}}{4\left|g^{\prime}\right|^{2}+\delta_{1}} \quad \text { on } V_{i, q}[0, \infty]=D_{\delta} \backslash\{0\},
$$

and we extend $\tilde{\rho}_{g}^{-2}|\mathbf{A}|^{2}$ on entire $D_{\delta}$ by this formula. Then, $\tilde{\rho}_{g}^{-2}|\mathbf{A}|^{2}$ is smooth on the entire $\bar{M}$. And, we define $\bar{\Omega}_{i}, \overline{\mathcal{C}}_{m}$ to be the closures of $\Omega_{i}, \mathcal{C}_{m}$ in $\bar{M}$, respectively. 
Definition 4.2.2. Given $\tilde{c}>0$, we define the " $\tilde{c}$-approximate kernel of $\Omega_{i}$ ( or $\mathcal{C}_{m}$ )" to be the span of those eigenfunctions of the Dirichlet problem for $\Delta_{h}+\tilde{\rho}_{g}^{-2}|\mathbf{A}|^{2}$ on $\bar{\Omega}_{i}\left(\right.$ or $\overline{\mathcal{C}}_{m}$ ) which have corresponding eigenvalues in $[-\tilde{c}, \tilde{c}]$.

Lemma 4.2.3. If $\delta, \delta_{1}$, and $\tau$ are small enough, then there exists $\tilde{c}>0$ such that the $\tilde{c}$-approximate kernel of $\Omega_{i}$ or $\mathcal{C}_{m}$ is 3 dimensional, and we can find a basis $f_{i, s}, \bar{f}_{m, s}$ for $1 \leq s \leq 3$ such that

$$
\left\|f_{i, s}-\vec{e}_{s} \cdot \vec{\nu}_{\zeta, \vec{\xi}}: L^{2}\left(\bar{\Omega}_{i}, h\right)\right\| \leq \tilde{c}, \quad\left\|\bar{f}_{m, s}-\vec{e}_{s} \cdot \vec{\nu}_{\zeta, \vec{\xi}}: L^{2}\left(\mathcal{C}_{m}, h\right)\right\| \leq \tilde{c}
$$

Moreover, we can take $\tilde{c}$ to be arbitrarily small by taking small enough $\delta, \delta_{1}, \tau$. We define $f_{i}:=\left(f_{i, 1}, f_{i, 2}, f_{i, 3}\right)$ and $\bar{f}_{m}:=\left(\bar{f}_{m, 1}, \bar{f}_{m, 2}, \bar{f}_{m, 3}\right)$.

Proof. The proof is basically an application of [K1, Appendix B], which is thus assumed to be familiar to the reader, and only the proof for $\Omega_{i}$ is provided since the proof for $\mathcal{C}_{m}$ is similar.

First we construct a Riemannian manifold which is to be compared with $\left(\bar{\Omega}_{i}, h\right)$. Define a smooth (nonsingular) metric $h, \tilde{\rho}_{g}$ on $\bar{M}_{i}$ in a similar manner to the construction of $h, \tilde{\rho}_{g}$ on $\bar{M}$ in definition 4.2.1, that is $h$ is a slight perturbation of $\frac{|\mathbf{A}|^{2}}{2} \mathbf{g}$ so that it is not singular at umbilic points and at ends. By our assumption of no exceptional bounded Jacobi fields, we see that the kernel of $\Delta_{h}+\tilde{\rho}_{g}^{-2}|\mathbf{A}|^{2}$ on $\bar{M}_{i}$ is 3-dimensional and spanned by $\vec{e}_{s} \cdot \vec{\nu}$, for $s=1,2,3$, where $\vec{\nu}$ is a unit normal vector field of $\tilde{X}_{i}\left(M_{i}\right)$, and that there exists some constant $c_{\bar{M}_{i}}>0$ such that there are no eigenvalues in $\left[-c_{\bar{M}_{i}}, c_{\bar{M}_{i}}\right]$ except 0 . Now, recall that for each $j$ such that $V_{j}\left[0, \ell_{j}\right] \subset \bar{\Omega}_{i}$, there is a catenoid which contains $V_{j}\left[\ell_{j} / 2, \ell_{j}\right]$ It is divided into two components by the circle $\mathcal{C}_{j}\left[\ell_{j}\right]$. Let $D_{j} \subset \mathbb{S}^{2}$ be the closure of the image by the Gauss map of the smaller component, and $h$ the standard metric of the sphere. We choose $\delta$ small enough that there are no low Dirichlet eigenvalues of $\Delta_{h}+2$, say less than 100. Define $\mathcal{L}_{h}$ on $\bar{M}_{i} \cup_{j} D_{j}$ to be $\Delta_{h}+\tilde{\rho}_{g}^{-2}|\mathbf{A}|^{2}$ on $\bar{M}_{i}$ and $\Delta_{h}+2$ on $D_{j}$. Then, the kernel of $\mathcal{L}_{h}$ is spanned also by $\vec{e}_{s} \cdot \vec{\nu}$ for $s=1,2,3$ and there are no eigenvalues in $\left[-c_{\bar{M}_{i}}, c_{\bar{M}_{i}}\right]$ except 0 .

Note that the assumptions (1),(2) of $[\mathrm{K} 1, \mathrm{~B} .1 .4]$ are satisfied because $\left(\bar{\Omega}_{i}, h\right)$ and $\left(\bar{M}_{i} \cup_{j} D_{j}, h\right)$ are compact. The use of assumption (3) of [K1,B.1.4] in finding the $C^{0}$ norm of eigenfunctions of low eigenvalues is replaced by the following argument: Let $f$ or $g$ be a Dirichlet eigenfunction of $\mathcal{L}_{h}$ on $\bar{M}_{i} \cup_{j} D_{j}$ or of $\Delta_{h}+\tilde{\rho}_{g}^{-2}|\mathbf{A}|^{2}$ on $\bar{\Omega}_{i}$, respectively, of low eigenvalue, 
say less than 10 . Then,

$$
\begin{aligned}
\left\|f: C^{0}\left(\bar{M}_{i} \cup_{j} D_{j}\right)\right\| & \leq C\left(\delta, \delta_{1}\right)\left\|f: L^{2}\left(\bar{M}_{i} \cup_{j} D_{j}, h\right)\right\|, \\
\left\|g: C^{0}\left(\bar{\Omega}_{i}\right)\right\| & \leq C\left(\delta, \delta_{1}\right)\left\|g: L^{2}\left(\bar{\Omega}_{i}, h\right)\right\| .
\end{aligned}
$$

The first inequality follows from standard theory. Note the dependency of the constant upon $\delta, \delta_{1}$, which follows from the dependency, for example, of the Sobolev constant upon them. For the second, we first note that

$$
\left\|g: C^{0}\left(\bar{\Omega}_{i} \backslash \cup_{j} V_{j}\left[2, \ell_{j}\right]\right)\right\| \leq C\left(\delta, \delta_{1}\right)\left\|g: L^{2}\left(\bar{\Omega}_{i}, h\right)\right\|
$$

from the uniform control on the geometry of $\left(\bar{\Omega}_{i} \backslash \cup_{j} V_{j}\left[2, \ell_{j}\right], h\right)$. To estimate on $V_{j}\left[2, \ell_{j}\right]$, we observe

$$
\left(\Delta_{\chi}+\rho_{g}^{-2}\left(\left(1+\frac{\lambda}{2}\right)|\mathbf{A}|^{2}+\frac{\lambda}{2} \delta_{1}\right)\right) g=0 \quad \text { in } V_{j}\left[2, \ell_{j}\right], \quad g=0 \quad \text { on } \mathcal{C}_{j}\left[\ell_{j}\right]
$$

Now, from lemma 3.3.3 we see that $\left(1+\frac{\lambda}{2}\right)|\mathbf{A}|^{2}+\frac{\lambda}{2} \delta_{1}$ satisfies the same estimate as $|\mathbf{A}|^{2}$, hence we can apply lemmas 4.1.2,4.1.5 with the above estimate to conclude

$$
\left\|g: C^{0}\left(V_{j}\left[2, \ell_{j}\right]\right)\right\| \leq C\left(\delta, \delta_{1}\right)\left\|g: L^{2}\left(\bar{\Omega}_{i}, h\right)\right\| .
$$

Now, we are going to construct maps

$$
\mathcal{F}: C_{0}^{\infty}\left(\bar{M}_{i} \cup_{j} D_{j}\right) \rightarrow C_{0}^{\infty}\left(\bar{\Omega}_{i}\right), \quad \mathcal{G}: C_{0}^{\infty}\left(\bar{\Omega}_{i}\right) \rightarrow C_{0}^{\infty}\left(\bar{M}_{i} \cup_{j} D_{j}\right)
$$

which will satisfy the assumption (4) of [K1,B.1.4].

First, we define $\mathcal{I}: \bar{\Omega}_{i} \rightarrow \bar{M}_{i} \cup_{j} D_{j}$ by sending each $V_{j}\left[\frac{\ell_{j}}{2}, \ell_{j}\right] \subset \Omega_{i}$ into $D_{j}$ using the Gauss map, by sending $V_{j}\left[\frac{\ell_{j}}{2}-1, \frac{\ell_{j}}{2}\right]$ into $\bar{M}_{i}$ using the projection maps into $P_{j}$ on the images of $\tilde{X}_{i}: M_{i} \rightarrow \mathbb{E}^{3}$ and $\vec{X}_{\zeta, \vec{\xi}}$, and by identifying $\bar{\Omega}_{i} \backslash \cup_{j} V_{j}\left[\frac{\ell_{j}}{2}-1, \ell_{j}\right]$ with the obvious domain in $\bar{M}_{i}$. Let $\rho: \mathbb{S}^{2} \rightarrow \mathbb{R}$ denote the distance function from $\cup_{j}\left\{e_{j}\right\} \subset \mathbb{S}^{2}$ where $e_{j}$ is the unit normal along the $z_{j}$ coordinate axis, and define a logarithmic cutoff function $\psi_{\mathbb{S}^{2}}: \mathbb{S}^{2} \rightarrow$ $\mathbb{R}$ by $\psi_{\mathbb{S}^{2}}(\vec{e}):=\psi[2,1]\left(\log \rho(\vec{e}) / \log \delta_{h}\right)$ where $\delta_{h}$ is a small constant to be determined later in this proof. Define smooth cutoff functions $\psi_{\bar{\Omega}_{i}}$ on $\bar{\Omega}_{i}$ to be $\psi_{\mathbb{S}^{2}} \circ \nu_{\zeta, \vec{\xi}}$ on $V_{j}\left[0, \ell_{j}\right]$ for each $j$ and 1 on $\bar{\Omega}_{i} \backslash \cup_{j} V_{j}\left[0, \ell_{j}\right]$, and $\psi_{\bar{M}_{i} \cup_{j} D_{j}}$ on $\bar{M}_{i} \cup_{j} D_{j}$ to be $\psi_{\mathbb{S}^{2}} \circ \vec{\nu}_{\zeta, \vec{\xi}}$ on $\cup_{j}\left(\mathcal{N}_{j} \cup D_{j}\right)$ for each $j$, where $\mathcal{N}_{j} \subset \bar{M}_{i}$ is the obvious neighborhood of $p_{j} \in \bar{M}_{i}$, and 1 elsewhere on $\bar{M}_{i} \cup_{j} D_{j}$. Finally we define $\mathcal{F}(f)$ to be the the pull-back of $\psi_{\bar{M}_{i} \cup_{j} D_{j}} f$ by $\mathcal{I}$, and $\mathcal{G}(g)$ to be the push-forward of $\psi_{\bar{\Omega}_{i}} g$ by $\mathcal{I}$. 
It is not hard to check that the assumption (4) is now satisfied, arguing as follows: The region on $\bar{\Omega}_{i}$ ( or on $\bar{M}_{i} \cup_{j} D_{j}$ ) where $\psi_{\bar{\Omega}_{i}}\left(\right.$ or $\psi_{\bar{M}_{i} \cup_{j} D_{j}}$ ) is not 1 has arbitrarily small $h$-area by choosing $\delta_{h}$ small enough ( regardless of $\delta, \delta_{1}$. This and the dependency upon $\delta, \delta_{1}$ of the constants in the bounds for the $C^{0}$ norm by $L^{2}$ norm are the reason for the introduction of $\cup_{j} D_{j}$ ). With $\delta_{h}$ chosen now, we can ensure that $\mathcal{I}$ is as close as we like to an isometry on the region where $\psi_{\bar{\Omega}_{i}}$ is not 0 , by choosing $\delta, \delta_{1}, \tau$ small enough. Note that the change in the $h$ metric on the perturbed end $V_{i, 1}[0, \infty]$ is small because of (4.1) and the smallness of the perturbation of the Gauss map which can be seen from (2.5), (2.6), (2.7).

However, in order to apply the proof of lemma [K1,B.2.2], we need extra conditions because the operators we are comparing are not exactly Laplacians. When we write $\mathcal{L}_{h}:=\Delta_{h}+B$, we should have

$$
\begin{aligned}
\left|\left\langle\tilde{\rho}^{-2}|\mathbf{A}|^{2} \mathcal{F}\left(f_{1}\right), \mathcal{F}\left(f_{2}\right)\right\rangle-\left\langle B f_{1}, f_{2}\right\rangle\right| & \leq \epsilon\left\|f_{1}\right\|_{\infty}\left\|f_{2}\right\|_{\infty}, \\
\left|\left\langle B \mathcal{G}\left(g_{1}\right), \mathcal{G}\left(g_{2}\right)\right\rangle-\left\langle\tilde{\rho}^{-2}|\mathbf{A}|^{2} g_{1}, g_{2}\right\rangle\right| & \leq \epsilon\left\|f_{1}\right\|_{\infty}\left\|f_{2}\right\|_{\infty}
\end{aligned}
$$

which are easy to check and left to the reader.

With the above setting, the proof of lemma [K1,B.2.2] can be easily adopted for the current lemma, with $\|f\|_{2}^{2}$ there replaced by $\left\langle\left(\mathcal{L}_{h}+\tilde{C}\right) f, f\right\rangle$ or $\left\langle\left(\Delta_{h}+\tilde{\rho}^{-2}|\mathbf{A}|^{2}+\tilde{C}\right) g, g\right\rangle$ for some constant $\tilde{C}$ which is smaller than the lowest Dirichlet eigenvalue of $\mathcal{L}_{h}$. Finally we have to compare $\mathcal{F}\left(\vec{\nu} \cdot \vec{e}_{s}\right)$ with $\vec{\nu}_{\zeta, \vec{\xi}} \cdot \vec{e}_{s}$ of $\Omega_{i}$, which is again left to the reader.

Definition 4.2.4. Define $\omega_{i, s}: \Omega_{i} \rightarrow \mathbb{R}$ and $\bar{\omega}_{m, s}: \mathcal{C}_{m} \rightarrow \mathbb{R}$ for $1 \leq s \leq 3$ by

$$
\omega_{i, s}(p):=\rho_{g}^{-2} \frac{\partial h_{\vec{\xi}, \Omega_{i}}}{\partial \xi_{\Omega_{i}, s}}(p), \quad \bar{\omega}_{m, s}(p):=\rho_{g}^{-2} \frac{\partial h_{\vec{\xi}, \mathcal{C}_{m}}}{\partial \xi_{\mathcal{C}_{m}, s}}(p) .
$$

Note that they are compactly supported. For the convenience of notation for later use, we let, where ${ }^{t}$ means the transpose,

$$
\omega_{i}:=\left(\omega_{i, 1}, \omega_{i, 2}, \omega_{i, 3}\right)^{t}, \quad \bar{\omega}_{m}:=\left(\bar{\omega}_{m, 1}, \bar{\omega}_{m, 2}, \bar{\omega}_{m, 3}\right)^{t} .
$$

Note that $h_{\vec{\xi}, \Omega_{i}}$ or $h_{\vec{\xi}, \mathcal{C}_{m}}$ is close to a linear combination of $\rho_{g}^{2} \omega_{i, s}$ 's or $\rho_{g}^{2} \bar{\omega}_{m, s}$ 's for $s=1,2,3$. The functions $\omega_{i, s}, \bar{\omega}_{m, s}$ play the role of the so-called substitute kernel. [K1,4-5].

Lemma 4.2.5. $\left\|\omega_{i, s}: C^{1}\left(\Omega_{i}, \chi\right)\right\| \leq C$ and $\left\|\bar{\omega}_{m, s}: C^{1}\left(\mathcal{C}_{m}, \chi\right)\right\| \leq C$. When

$$
K_{i}:=\left[\int_{\Omega_{i}} \rho_{g}^{2} \omega_{i, s} f_{i, t} d \mathbf{g}\right]_{s, t=1}^{3}, \quad \bar{K}_{m}:=\left[\int_{\mathcal{C}_{m}} \rho_{g}^{2} \bar{\omega}_{m, s} \bar{f}_{m, t} d \mathbf{g}\right]_{s, t=1}^{3},
$$


we have $\max _{1 \leq s, t \leq 3}\left\{\left|\left(K_{i}\right)_{s, t}-\delta_{s, t}\right|,\left|\left(\bar{K}_{m}\right)_{s, t}-\delta_{s, t}\right|\right\} \leq \tilde{\varepsilon}$, where $\tilde{\varepsilon}$ can be taken arbitrarily small when $\delta$ is small enough.

Proof. The estimates for $\omega_{i, s}$ 's and $\bar{\omega}_{m, s}$ 's follow easily from definitions. For $K_{i}$, note

$$
\int_{\Omega_{i}} \rho_{g}^{2} \omega_{i, s} f_{i, t} d \mathbf{g}=\int_{\Omega_{i}} \rho_{g}^{2} \omega_{i, s} \vec{e}_{t} \cdot \vec{\nu}_{\zeta, \vec{\xi}} d \mathbf{g}+\int_{\Omega_{i}} \rho_{g}^{2} \omega_{i, s}\left(f_{i, t}-\vec{e}_{t} \cdot \vec{\nu}_{\zeta, \vec{\xi}}\right) d \mathbf{g} .
$$

Now, we observe that

$$
\int_{\Omega_{i}} \rho_{g}^{2} \omega_{i, s} \vec{e}_{t} \cdot \vec{\nu}_{\zeta, \bar{\xi}} d \mathbf{g}=\frac{\partial}{\partial \xi_{i, s}} \int_{\Omega_{i}} h_{\vec{\xi}, \Omega_{i}} \vec{e}_{t} \cdot \vec{\nu}_{\zeta, \bar{\xi}} d \mathbf{g}=\frac{\partial \xi_{i, t}}{\partial \xi_{i, s}}=\delta_{s, t}
$$

where the second equality comes from the balancing formula [S2], [KKS]. We finish the proof by appealing to lemma 4.2.3. The proof for $\bar{K}_{m}$ is similar to the above and is omitted.

Lemma 4.2.6. There exists $\tilde{c}>0$ such that for any $E$ in $C_{\text {loc }}^{0, \alpha}\left(\Omega_{i}, \chi\right)$, there exists a unique $\Lambda \in \mathbb{R}^{3}$ such that $\rho_{g}^{2} \tilde{\rho}_{g}^{-2}\left(E+\Lambda \cdot \omega_{i}\right)$ is $L^{2}\left(\bar{\Omega}_{i}, h\right)$-orthogonal to the $\tilde{c}$-approximate kernel of $\Omega_{i}$, and also for any $E$ in $C_{l o c}^{0, \alpha}\left(\mathcal{C}_{m}, \chi\right)$, there exists unique $\bar{\Lambda} \in \mathbb{R}^{3}$ such that $\rho_{g}^{2} \tilde{\rho}_{g}^{-2}\left(E+\bar{\Lambda} \cdot \bar{\omega}_{i}\right)$ is $L^{2}\left(\overline{\mathcal{C}}_{m}, h\right)$-orthogonal to the $\tilde{c}$-approximate kernel of $\mathcal{C}_{m}$. In fact,

$$
\Lambda=-\int_{\Omega_{i}} \rho_{g}^{2} \tilde{\rho}_{g}^{-2} E f_{i} d h \cdot K_{i}^{-1}, \quad \bar{\Lambda}=-\int_{\mathcal{C}_{m}} \rho_{g}^{2} \tilde{\rho}_{g}^{-2} E \bar{f}_{m} d h \cdot \bar{K}_{m}^{-1}
$$

where $f_{i}$ and $\bar{f}_{m}$ are as in lemma 4.2.3. Moreover, if suppE $\subset \Omega_{i}^{\prime}$ or $\subset \mathcal{C}_{m}^{\prime}$, then

$$
|\Lambda| \leq C\left\|E: C^{0, \alpha}\left(\Omega_{i}, \chi\right)\right\|, \quad|\bar{\Lambda}| \leq C\left\|E: C^{0, \alpha}\left(\mathcal{C}_{m}, \chi\right)\right\| .
$$

Proof. We prove only the case $E \in C_{l o c}^{0, \alpha}\left(\Omega_{i}, \chi\right)$ since the proof for the other case is similar. Given $\mathrm{E}$, define $\Lambda$ by the formula given in the statement of the lemma. Then, we have

$$
\begin{array}{r}
\int_{\Omega_{i}} \rho_{g}^{2} \tilde{\rho}_{g}^{-2}\left(E+\Lambda \cdot \omega_{i}\right) f_{i, t} d h=\int_{\Omega_{i}} \rho_{g}^{2} \tilde{\rho}_{g}^{-2} E f_{i, t} d h+\Lambda \cdot \int_{\Omega_{i}} \rho_{g}^{2} \tilde{\rho}_{g}^{-2} \omega_{i} f_{i, t} d h \\
=\int_{\Omega_{i}} \rho_{g}^{2} \tilde{\rho}_{g}^{-2} E f_{i, t} d h-\int_{\Omega_{i}} \rho_{g}^{2} \tilde{\rho}_{g}^{-2} E f_{i} d h \cdot K_{i}^{-1} \cdot \int_{\Omega_{i}} \rho_{g}^{2} \tilde{\rho}_{g}^{-2} \omega_{i} f_{i, t} d h
\end{array}
$$


Now, we see that $\int_{\Omega_{i}} \rho_{g}^{2} \tilde{\rho}_{g}^{-2} \omega_{i} f_{i, t} d h=\int_{\Omega_{i}} \rho_{g}^{2} \omega_{i} f_{i, t} d \mathrm{~g}$ is the $t$-th column of $K_{i}$, hence $K_{i}^{-1} \cdot \int_{\Omega_{i}} \rho_{g}^{2} \tilde{\rho}_{g}^{-2} \omega_{i} f_{i, t} d h$ is a column vector whose entries are all zeros except the $t$-th row, which is 1 . Hence we get $\int_{\Omega_{i}} \rho_{g}^{2} \tilde{\rho}_{g}^{-2}\left(E+\Lambda \cdot \omega_{i}\right) f_{i, t} d h=0$. The uniqueness of such $\Lambda$ is obvious. The estimate for $\Lambda$ follows from lemmas $3.3 .4,4.2 .3,4.2 .5$ and various definitions.

Definition 4.2.7. Let $\bar{v}_{j}$ to be as in proposition 4.1.4, and for $\Omega_{i}$ which contains $V_{j}\left[0, \ell_{j}\right]$, define $v_{j}: \Omega_{i} \rightarrow \mathbb{R}$ to be the trivial extension of

$$
\bar{v}_{j} \psi\left[\frac{\ell}{2}+1, \frac{\ell}{2}+2\right] \circ x .
$$

Finally, $\eta_{j}:=\mathcal{L}_{\chi} v_{j}$. We have trivially

$$
\left\|v_{j}: C^{2, \alpha}\left(V_{j}\left[\frac{\ell_{j}}{2}, \frac{\ell_{j}}{2}+1\right], \chi\right)\right\| \leq C .
$$

Now we define some norms which are conveniently used in the rest of the paper.

Definition 4.2.8. Let $\tilde{q}$ be a continuous function which is $:=e^{-(1-2 \lambda)\left(x-\frac{\ell_{j}}{2}\right)}$ on $V_{j}\left[\ell_{j} / 2, \ell_{j}\right]$ and which is constant on each component of $M \backslash \bigcup_{j} V_{j}\left[\ell_{j} / 2, \ell_{j}\right]$. Then

$$
\begin{aligned}
\|\phi\|_{2} & :=\left\|\phi: C^{2, \alpha}(M, \chi, \tilde{q})\right\|, \\
\|E\|_{0} & :=\left\|E: C^{0, \alpha}\left(M, \chi, e^{-(1-2 \lambda) x / 2}\right)\right\| .
\end{aligned}
$$

Now we present how to obtain a global solution of the linearized equation with an arbitrary inhomogeneous term.

Proposition 4.2.9. Suppose $\|E\|_{0}<\infty$. Then, there are $\phi_{E} \in C_{l o c}^{2, \alpha}(M, \chi)$, $\Theta_{E, j} \in \mathbb{R}, \Lambda_{E, i}, \bar{\Lambda}_{E, m} \in \mathbb{R}^{3}$, unique by the construction, such that

1. $\mathcal{L}_{\chi} \phi_{E}=E+\sum \Theta_{E, j} \eta_{j}+\sum \Lambda_{E, i} \cdot \omega_{i}+\sum \bar{\Lambda}_{E, m} \cdot \bar{\omega}_{m}$ in $M$,

2. $\left\|\phi_{E}\right\|_{2} \leq C\|E\|_{0}$,

3. $\left|\Theta_{E, j}\right| \leq C\|E\|_{0}, \quad\left|\Lambda_{E, i}\right| \leq C\|E\|_{0}, \quad\left|\bar{\Lambda}_{E, m}\right| \leq C \tau^{(1-2 \lambda) / 2}\|E\|_{0}$.

Proof. Define smooth cutoff functions 
1. $\psi_{j}$ to be $\psi[1,2] \circ x-\psi\left[\ell_{j}-2, \ell_{j}-1\right] \circ x$ on $V_{j}\left[0, \ell_{j}\right]$, and vanish on $M \backslash V_{j}\left[0, \ell_{j}\right]$,

2. $\psi_{i, q}$ to be $\psi[1,2] \circ x$ on $V_{i, q}[0, \infty]$, and vanish on $M \backslash V_{i, q}[0, \infty]$,

3. $\tilde{\psi}_{k}$ to be $\psi\left[\ell_{k}+1, \ell_{k}+2\right] \circ x$ on $\tilde{V}_{k}\left[\ell_{k}, \infty\right]$, and vanish on $M \backslash \tilde{V}_{k}\left[\ell_{k}, \infty\right]$.

Let $\varphi_{j} \in C_{l o c}^{2, \alpha}\left(V_{j}\left[0, \ell_{j}\right], \chi\right), \varphi_{i, q} \in C_{l o c}^{2, \alpha}\left(V_{i, q}[0, \infty], \chi\right), \tilde{\varphi}_{k} \in C_{l o c}^{2, \alpha}\left(\tilde{V}_{k}\left[\ell_{k}, \infty\right], \chi\right)$ be given by $\mathcal{R}_{1}$ of proposition 4.1 .3 with $\psi_{j} E, \psi_{i, q} E, \tilde{\psi}_{k} E$, respectively, with appropriate $x_{0}, x_{1}$ and $\lambda_{0}=(1-2 \lambda) / 2$. Then,

$$
\left\|\psi_{j} \varphi_{j}\right\|_{2}, \quad\left\|\psi_{i, q} \varphi_{i, q}\right\|_{2}, \quad \tau^{-(1-2 \lambda) / 2}\left\|\tilde{\psi}_{k} \tilde{\varphi}_{k}\right\|_{2} \leq C\|E\|_{0}
$$

Define

$$
\begin{aligned}
E_{0}:= & -\sum\left[\mathcal{L}_{\chi}, \psi_{i, q}\right] \varphi_{i, q}-\sum\left[\mathcal{L}_{\chi}, \tilde{\psi}_{k}\right] \tilde{\varphi}_{k}-\sum\left[\mathcal{L}_{\chi}, \psi_{j}\right] \varphi_{j} \\
& +\left(1-\sum \psi_{i, q}^{2}-\sum \tilde{\psi}_{k}^{2}-\sum \psi_{j}^{2}\right) E
\end{aligned}
$$

Then,

$$
\begin{gathered}
\mathcal{L}_{\chi}\left(\sum \psi_{i, q} \varphi_{i, q}+\sum \tilde{\psi}_{k} \tilde{\varphi}_{k}+\sum \psi_{j} \varphi_{j}\right)=E-E_{0}, \\
\left\|E_{0}\right\|_{0} \leq C\|E\|_{0}, \quad \operatorname{supp} E \subset \cup \Omega_{i}^{\prime} \bigcup \cup \mathcal{C}_{m}^{\prime} .
\end{gathered}
$$

Decompose $E_{0}$ such that $E_{0}=\sum E_{\Omega_{i}}+\sum E_{\mathcal{C}_{m}}$ with $\operatorname{supp} E_{\Omega_{i}} \subset \Omega_{i}^{\prime}$ and $\operatorname{supp} E_{\mathcal{C}_{m}} \subset \mathcal{C}_{m}^{\prime}$. Then,

$\left\|E_{\Omega_{i}}: C^{0, \alpha}\left(\Omega_{i}, \chi\right)\right\| \leq C\|E\|_{0}, \quad\left\|E_{\mathcal{C}_{m}}: C^{0, \alpha}\left(\mathcal{C}_{m}, \chi\right)\right\| \leq C \tau^{(1-2 \lambda) / 2}\|E\|_{0}$.

Let $\phi_{\Omega_{i}}: \bar{\Omega}_{i} \rightarrow \mathbb{R}, \Lambda_{\Omega_{i}, 1} \in \mathbb{R}^{3}$ and $\phi_{\mathcal{C}_{m}}: \mathcal{C}_{m} \rightarrow \mathbb{R}, \bar{\Lambda}_{\mathcal{C}_{m}, 1} \in \mathbb{R}^{3}$ be given by lemma 4.2 .6 with $E_{\Omega_{i}}$ and $E_{\mathcal{C}_{m}}$ respectively. Then

(a)

$$
\begin{aligned}
\left(\Delta_{h}+\tilde{\rho}_{g}^{-2}|\mathbf{A}|^{2}\right) \phi_{\Omega_{i}} & =\rho_{g}^{2} \tilde{\rho}_{g}^{-2}\left(E_{\Omega_{i}}+\Lambda_{\Omega_{i}, 1} \cdot \omega_{j}\right) \text { in } \bar{\Omega}_{i}, & \phi_{\Omega_{i}} & =0 \text { on } \partial \bar{\Omega}_{i} \\
\left(\Delta_{h}+\tilde{\rho}_{g}^{-2}|\mathbf{A}|^{2}\right) \phi_{\mathcal{C}_{m}} & =\rho_{g}^{2} \tilde{\rho}_{g}^{-2}\left(E_{\mathcal{C}_{m}}+\bar{\Lambda}_{\mathcal{C}_{m}, 1} \cdot \bar{\omega}_{m}\right) \text { in } \overline{\mathcal{C}}_{m}, & \phi_{\mathcal{C}_{m}} & =0 \text { on } \partial \overline{\mathcal{C}}_{m}
\end{aligned}
$$

(b) $\left|\Lambda_{\Omega_{i}, 1}\right| \leq C\left\|E_{0}\right\|_{0}, \quad\left|\bar{\Lambda}_{\mathcal{C}_{m}, 1}\right| \leq C \tau^{(1-2 \lambda) / 2}\left\|E_{0}\right\|_{0}$. 
We obtain the $C^{0}\left(\Omega_{i} \backslash \cup V_{j}\left[2, \ell_{j}\right]\right)$ bound of $\phi_{\Omega_{i}}$ and $C^{0}\left(\mathcal{C}_{m} \backslash \cup V_{j}\left[0, \ell_{j}-2\right]\right)$ bound of $\phi_{C_{m}}$ from (a),(b) and standard theory, and the $C^{0}$ bounds on $\cup_{j} V_{j}\left[0, \ell_{j}\right]$ by lemmas 4.1.2,4.1.5 after rewriting (a) in terms of $\mathcal{L}_{\chi}$. Hence by standard theory

$$
\left\|\phi_{\Omega_{i}}: C^{2, \alpha}\left(\Omega_{i}, \chi\right)\right\| \leq C\left\|E_{0}\right\|_{0}, \quad\left\|\phi_{\mathcal{C}_{m}}: C^{2, \alpha}\left(\mathcal{C}_{m}, \chi\right)\right\| \leq C \tau^{(1-2 \lambda) / 2}\left\|E_{0}\right\|_{0} .
$$

Let $\Theta_{j, 1} \in \mathbb{R}$ be given by proposition 4.1 .4 with $\phi_{\Omega_{i}}$ restricted to $V_{j}\left[\frac{\ell_{j}}{2}, \ell_{j}\right]$. Then

$$
\left|\Theta_{j, 1}\right| \leq C\left\|E_{0}\right\|_{0}, \quad\left\|\left(\phi_{\Omega_{i}}+\sum \Theta_{j, 1} v_{j}\right) \psi_{\Omega_{i}}\right\|_{2} \leq C\left\|E_{0}\right\|_{0} .
$$

where the sum is for all $j$ such that $V_{j}\left[0, \ell_{j}\right] \subset \Omega_{i}$. Let

1. $\psi_{\Omega_{i}}:=1$ on $\Omega_{i} \backslash \cup_{j} V_{j}\left[0, \ell_{j}\right],:=\psi\left[\ell_{j}-1, \ell_{j}-2\right] \circ x$ in $V_{j}\left[0, \ell_{j}\right] \subset \Omega_{i},:=$ 0 in $M \backslash \Omega_{i}$,

2. $\psi_{\mathcal{C}_{m}}:=1$ on $\mathcal{C}_{m} \backslash \cup_{j} V_{j}\left[0, \ell_{j}\right],:=\psi[1,2] \circ x$ in $V_{j}\left[0, \ell_{j}\right] \subset \mathcal{C}_{m},:=0$ on $M \backslash \mathcal{C}_{m}$

and define

$$
\begin{aligned}
E_{1} & :=-\sum\left[\mathcal{L}_{\chi}, \psi_{\Omega_{i}}\right]\left(\phi_{\Omega_{i}}+\sum \Theta_{j, 1} v_{j}\right)-\sum\left[\mathcal{L}_{\chi}, \psi_{\mathcal{C}_{m}}\right] \phi_{\mathcal{C}_{m}}, \\
\varphi_{1} & :=\sum \psi_{\Omega_{i}}\left(\phi_{\Omega_{i}}+\sum \Theta_{j, 1} v_{j}\right)+\sum \psi_{\mathcal{C}_{m}} \phi_{\mathcal{C}_{m}} .
\end{aligned}
$$

Then, $\operatorname{supp} E_{1} \subset \cup \Omega_{i}^{\prime} \bigcup \cup \mathcal{C}_{m}^{\prime}$, and

$$
\left\|E_{1}\right\|_{0} \leq C \tau^{\lambda / 2}\left\|E_{0}\right\|_{0}, \quad\left\|\varphi_{1}\right\|_{2} \leq C\left\|E_{0}\right\|_{0} .
$$

(Note that the factor $\tau^{\lambda / 2}$ is obtained from the the difference of decay rates in proposition 4.1.4 and definition 4.2.8). Define inductively $E_{n+1}, \varphi_{n+1}, \Theta_{i, n+1}, \Lambda_{j, n+1}$ from $E_{n}$ as we get $E_{1}, \varphi_{1}, \Theta_{i, 1}, \Lambda_{j, 1}$ from $E_{0}$. Then, for any $n \geq 1$,

$$
\begin{aligned}
& \mathcal{L}_{\chi} \varphi_{n}=E_{n-1}-E_{n}+\sum \Theta_{j, n} \eta_{j}+\sum \Lambda_{i, n} \cdot \omega_{i}+\sum \bar{\Lambda}_{m, n} \cdot \bar{\omega}_{m} \quad \text { in } M, \\
& \left\|E_{n-1}-E_{n}\right\|_{0} \leq 2\left\|E_{n-1}\right\|_{0} \leq 2\left(C \tau^{\lambda / 2}\right)^{n-1}\left\|E_{0}\right\|_{0}, \\
& \left\|\varphi_{n}\right\|_{2} \leq C\left\|E_{n-1}\right\|_{0} \leq C\left(C \tau^{\lambda / 2}\right)^{n-1}\left\|E_{0}\right\|_{0} \\
& \left|\Theta_{j, n}\right|,\left|\Lambda_{i, n}\right| \leq C\left\|E_{n-1}\right\|_{0} \leq C\left(C \tau^{\lambda / 2}\right)^{n-1}\left\|E_{0}\right\|_{0} \\
& \left|\bar{\Lambda}_{m, n}\right| \leq C \tau^{(1-2 \lambda) / 2}\left\|E_{n-1}\right\|_{0} \leq C \tau^{(1-2 \lambda) / 2}\left(C \tau^{\lambda / 2}\right)^{n-1}\left\|E_{0}\right\|_{0} .
\end{aligned}
$$


Define

$$
\begin{array}{r}
\phi_{E}:=\sum \psi_{i, q} \varphi_{i, q}+\sum \tilde{\psi}_{k} \tilde{\varphi}_{k}+\sum \psi_{j} \varphi_{j}+\sum_{n=1}^{\infty} \varphi_{n} \\
\Theta_{E, j}:=\sum_{n=1}^{\infty} \Theta_{j, n}, \quad \Lambda_{E, i}:=\sum_{n=1}^{\infty} \Lambda_{i, n}, \quad \bar{\Lambda}_{E, m}:=\sum_{n=1}^{\infty} \bar{\Lambda}_{m, n} .
\end{array}
$$

\subsection{Linearized minimal surface equation.}

Lemma 4.3.1. For any parameter $\left(\left(\zeta_{j}\right),\left(\vec{\xi}_{\Omega_{i}}\right),\left(\vec{\xi}_{\mathcal{C}_{m}}\right)\right) \in Z_{\gamma_{1}} \times \Xi_{\gamma_{2}} \times \bar{\Xi}_{\gamma_{2}}$, there are $\phi_{\tau} \in C_{\text {loc }}^{2, \alpha}(M, \chi), \Theta_{\tau, j} \in \mathbb{R}, \Lambda_{\tau, i} \in \mathbb{R}^{3}, \bar{\Lambda}_{\tau, m} \in \mathbb{R}^{3}$, unique by the construction, such that

1. $\mathcal{L}_{\chi} \phi_{\tau}=-2 \rho_{g}^{-2} \sum h_{\tau, j}+\sum \Lambda_{\tau, i} \cdot \omega_{i}+\sum \bar{\Lambda}_{\tau, m} \cdot \bar{\omega}_{m}+\sum \Theta_{\tau, j} \eta_{j}$,

2. $\left\|\phi_{\tau}\right\|_{2} \leq C \ell^{2} \tau$,

3. $\left|\Theta_{\tau, j}\right| \leq C \ell^{2} \tau, \quad\left|\Lambda_{\tau, i}\right| \leq C \ell^{2} \tau, \quad\left|\bar{\Lambda}_{\tau, m}\right| \leq C \ell^{2} \tau^{(3-2 \lambda) / 2}$.

Proof. By lemma 4.1.1, we have $\phi_{j}$ such that

$$
\mathcal{L}_{\chi} \phi_{j}=-2 \rho_{g}^{-2} h_{\tau, j} \quad \text { in } V_{j}\left[0, \ell_{j}\right], \quad \phi_{j}=0 \quad \text { on } \partial V_{j}\left[0, \ell_{j}\right] .
$$

Then, standard theory and lemma 4.1.1 imply

$$
\left\|\phi_{j}: C^{2, \alpha}\left(V_{j}\left[0, \ell_{j}\right], \chi\right)\right\| \leq C \ell_{j}^{2} \tau_{j} .
$$

Now, proposition 4.1.4 and definition 4.2 .7 give $a_{j} \in \mathbb{R}$ such that $\left\|\phi_{j}+a_{j} v_{j}: C^{2, \alpha}\left(V_{j}\left[\ell_{j} / 2, \ell_{j}\right], \chi, e^{-(1-\lambda)\left(x-\ell_{j} / 2\right)}\right)\right\| \leq C \ell_{j}^{2} \tau_{j}, \quad\left|a_{j}\right| \leq C \ell_{j}^{2} \tau_{j}$.

If we let

$$
E:=-\sum\left[\mathcal{L}_{\chi}, \psi[1,2] \circ x-\psi[\ell-2, \ell-1] \circ x\right]\left(\phi_{j}+a_{j} v_{j}\right),
$$

then $\|E\|_{0} \leq C \ell^{2} \tau$. Let $\phi_{E}, \Theta_{E, j}, \Lambda_{E, i}, \bar{\Lambda}_{E, m}$ be given by proposition 4.2 .9 with the above $E$, and define

$$
\begin{aligned}
\phi_{\tau} & :=\sum(\psi[1,2] \circ x-\psi[\ell-2, \ell-1] \circ x)\left(\phi_{j}+a_{j} v_{j}\right)+\phi_{E} \\
\Theta_{\tau, j} & :=a_{j}+\Theta_{E, j}, \quad \Lambda_{\tau, i}:=\Lambda_{E, i}, \quad \bar{\Lambda}_{\tau, m}:=\bar{\Lambda}_{E, m} .
\end{aligned}
$$


Lemma 4.3.2. For any parameter $\left(\left(\zeta_{j}\right),\left(\vec{\xi}_{\Omega_{i}}\right),\left(\vec{\xi}_{\mathcal{C}_{m}}\right)\right) \in Z_{\gamma_{1}} \times \Xi_{\gamma_{2}} \times \bar{\Xi}_{\gamma_{2}}$, there are $\phi_{\zeta} \in C_{\text {loc }}^{2, \alpha}(M, \chi), \Theta_{\zeta, j} \in \mathbb{R}, \Lambda_{\zeta, i} \in \mathbb{R}^{3}, \bar{\Lambda}_{\zeta, m} \in \mathbb{R}^{3}$, unique by the construction, such that
1. $\mathcal{L}_{\chi} \phi_{\zeta}=-2 \rho_{g}^{-2} \sum h_{\zeta, i}+\sum \Lambda_{\zeta, i} \cdot \omega_{i}+\sum \bar{\Lambda}_{\zeta, m} \cdot \bar{\omega}_{m}+\sum \Theta_{\zeta, j} \eta_{j}$,
2. $\left\|\phi_{\zeta}\right\|_{2} \leq C \gamma_{1} \ell^{2} \tau$,
3. $\left|\zeta_{j}-\Theta_{\zeta, j}\right| \leq \frac{2}{3} \gamma_{1} \ell^{2} \tau, \quad\left|\Lambda_{\zeta, i}\right| \leq C \gamma_{1} \ell \tau, \quad\left|\bar{\Lambda}_{\zeta, m}\right| \leq C \gamma_{1} \ell \tau^{(3-2 \lambda) / 2}$.

Proof. Recall that for each joining piece $V_{j}\left[0, \ell_{j}\right]$, there is associated the cylindrical coordinates system $\left(r_{j}, \theta_{j}, z_{j}\right)$. For the convenience of notation in this proof, we define $\tilde{x}_{j}: \mathcal{N}_{j} \cap \cup_{i=1}^{I+1} \Omega_{i} \rightarrow \mathbb{R}$ by $\tilde{x}_{j}(p):=x(p)$ in $V_{j}\left[0, \ell_{j}\right]$ and $:=1-r_{i} \circ \vec{X}_{\zeta, \vec{\xi}}(p) / \delta$ elsewhere. Let $\tilde{V}_{j}[a, b]$ be defined as in definition 3.2.2 but with $\tilde{x}_{j}$ instead of $x$. Note that $\tilde{V}_{j}[a, b]=V_{j}[a, b]$ for any $0 \leq a \leq b \leq \ell_{j}$.

Let $\phi_{j, 1}: \tilde{V}_{j}\left[-3, \ell_{j}\right] \rightarrow \mathbb{R}$ be such that the image of $\vec{X}_{\zeta, \vec{\xi}}+\phi_{j, 1} \vec{\nu}_{\zeta, \vec{\xi}}$ belongs to $\vec{X}_{\zeta, \vec{\xi}}(M)$ with $\zeta_{j}=0$. Then

(a) $\left\|\phi_{j, 1}: C^{3}\left(\tilde{V}_{j}[-3,-1], \chi\right)\right\| \leq C\left|\zeta_{j}\right|$ and $\phi_{j, 1}$ vanishes on $\tilde{V}_{j}\left[-1, \ell_{j}\right]$,

(b) $\left\|\phi_{j, 1}-\zeta_{j} \vec{e}_{j} \cdot \vec{\nu}_{\zeta, \vec{\xi}}: C^{3}\left(\tilde{V}_{j}[-3,-2], \chi\right)\right\| \leq C\left|\zeta_{j}\right|^{2}$,

(c) $\left\|\mathcal{L}_{\chi} \phi_{j, 1}+2 \rho_{g}^{-2} h_{\zeta, j}: C^{1}\left(\tilde{V}_{j}[-2,-1], \chi\right)\right\| \leq C\left|\zeta_{j}\right|^{2}$ since it has only higher order terms in $\phi_{j, 1}$ (recall the mean curvature identity (1.1), and note that $\rho_{g} \equiv 1$ in this region.),

where $\vec{e}_{j}$ is the unit vector in the positive $z_{j}$ direction (up to sign). Now, note that $\mathcal{L}_{\chi}\left(\vec{e}_{j} \cdot \vec{\nu}_{\zeta, \vec{\xi}}\right)=0$ on $\tilde{V}_{j}[-3,-2] \cup \tilde{V}_{j}\left[-1, \frac{\ell_{j}}{2}-1\right] \cup \tilde{V}_{j}\left[\frac{\ell_{j}}{2}, \ell_{j}\right]$ and that $\vec{e}_{j} \cdot \vec{\nu}_{\zeta, \xi}$ is $1 / \sqrt{1+\delta^{-2} e^{2 x}\left(\partial_{x} \Phi_{j}^{2}+\partial_{\theta} \Phi_{j}^{2}\right)}$ in $\tilde{V}_{j}\left[0, \ell_{j}\right]$. By utilizing (3.6), lemmas $3.3 .1,3.3 .2$, corollary 3.3 .5 , and the uniform geometry on $\tilde{V}_{j}[-3,0]$, we have

(d) $\left\|\vec{e}_{j} \cdot \vec{\nu}_{\zeta, \vec{\xi}}-1: C^{0}\left(\tilde{V}_{j}\left[-3, \ell_{j}\right]\right)\right\| \leq \tilde{\varepsilon}, \quad\left\|\vec{e}_{j} \cdot \vec{\nu}_{\zeta, \vec{\xi}}: C^{3}\left(\tilde{V}_{j}\left[-3, \ell_{j}\right], \chi\right)\right\| \leq C$.

(e) $\left\|\mathcal{L}_{\chi}\left(\vec{e}_{j} \cdot \vec{\nu}_{\zeta, \vec{\xi}}\right): C^{1}\left(\tilde{V}_{j}[-2,-1], \chi\right)\right\| \leq C\left|\zeta_{j}\right|$. (Note that $\mathcal{L}_{\chi}\left(\vec{e}_{j} \cdot \vec{\nu}_{\zeta, \vec{\xi}}\right)=0$ in this region when $\zeta_{j}=0$.)

(f) $\left\|\mathcal{L}_{\chi}\left(\vec{e}_{j} \cdot \vec{\nu}_{\zeta, \vec{\xi}}\right): C^{1}\left(\tilde{V}_{j}\left[\ell_{j} / 2-1, \ell_{j} / 2\right], \chi\right)\right\| \leq C \tau$ from explicit computations. 
For $\tilde{V}_{j}\left[0, \ell_{j}\right] \subset \Omega_{i}$, define $\phi_{j, 2}, E_{j, 1}, E_{j, 2}: \Omega_{i} \rightarrow \mathbb{R}$ by $\phi_{j, 2}:=\left(\phi_{j, 1}-\zeta_{j} \vec{e}_{j} \cdot \vec{\nu}_{\zeta, \vec{\xi}}\right) \psi[-3,-2] \circ \tilde{x}_{j}, \quad \mathcal{L}_{\chi} \phi_{j, 2}+2 \rho_{g}^{-2} h_{\zeta, j}:=E_{j, 1}+E_{j, 2}$ with $\operatorname{supp} E_{j, 1} \in \tilde{V}_{j}[-3,-1], \operatorname{supp} E_{j, 2} \in \tilde{V}_{j}\left[\ell_{j} / 2-1, \ell_{j} / 2\right]$. Then,

(g) $\left\|E_{j, 1}: C^{1}\left(\tilde{V}_{j}[-3,-1], \chi\right)\right\| \leq C\left|\zeta_{j}\right|^{2}$ from (b),(c), and (e).

By propositions 4.1.1,4.1.3, and standard theory, we have $\phi_{j, 3}, \phi_{j, 4}$ : $\tilde{V}_{j}\left[0, \ell_{j}\right] \rightarrow \mathbb{R}$ such that

$$
\begin{aligned}
\mathcal{L}_{\chi} \phi_{j, 3} & =-E_{j, 2} \quad \text { in } \tilde{V}_{j}\left[0, \ell_{j}\right] \\
\phi_{j, 3} & =0 \quad \text { on } \mathcal{C}_{j}[0] \cup \mathcal{C}_{j}\left[\ell_{j}\right] \\
\mathcal{L}_{\chi} \phi_{j, 4} & =0 \quad \text { in } \tilde{V}_{j}\left[0, \ell_{j}\right] \\
\phi_{j, 4} & =0 \quad \text { on } \mathcal{C}_{j}[0], \\
\phi_{j, 4} & =\zeta_{j} \vec{e}_{j} \cdot \vec{\nu}_{\zeta, \vec{\xi}} \quad \text { on } \mathcal{C}_{j}\left[\ell_{j}\right] .
\end{aligned}
$$

$\phi_{j, 3}, \phi_{j, 4}$ satisfy

(h) $\left\|\phi_{j, 3}: C^{2, \alpha}\left(\tilde{V}_{j}\left[0, \ell_{j}\right], \chi\right)\right\| \leq C \ell^{2} \tau\left|\zeta_{j}\right|$ by (f), lemma 4.1.1, and standard theory,

(i) $\left|\phi_{j, 4}-\zeta_{j} \cdot x / \ell\right| \leq \tilde{\varepsilon}\left|\zeta_{j}\right|$ by lemmas 4.1.2,4.1.5, and (d).

Now we observe

$\mathcal{L}_{\chi}\left(\phi_{j, 2}+\phi_{j, 3}+\phi_{j, 4}\right)=0 \quad$ in $\tilde{V}_{j}\left[\ell_{j} / 2, \ell_{j}\right], \quad \phi_{j, 2}+\phi_{j, 3}+\phi_{j, 4}=0 \quad$ on $\mathcal{C}_{j}\left[\ell_{j}\right]$ and

(j) $\left|\zeta_{j} / 2-\underset{\mathcal{C}\left[\ell_{j} / 2\right]}{\operatorname{avg}}\left(\phi_{j, 2}+\phi_{j, 3}+\phi_{j, 4}\right)\right| \leq C \tilde{\varepsilon}\left|\zeta_{j}\right|$ from (d),(h), and (i).

Let $a_{j} \in \mathbb{R}$ be given by proposition 4.1 .4 with $\phi=\phi_{j, 2}+\phi_{j, 3}+\phi_{j, 4}$. Then

$$
\left|a_{j}-\zeta_{j}\right| \leq \frac{5}{8}\left|\zeta_{j}\right|
$$

by (j). If we define $\phi_{j, 5}: \Omega_{i} \rightarrow \mathbb{R}$ by

$$
\phi_{j, 5}:=\phi_{j, 2}+\left(\phi_{j, 3}+\phi_{j, 4}\right) \psi[1,2] \circ x+a_{j} v_{j},
$$

then, $\left\|\phi_{j, 5} \psi\left[\ell_{j}-2, \ell_{j}-1\right] \circ x\right\|_{2} \leq C\left|\zeta_{j}\right|$ and

$$
\mathcal{L}_{\chi} \phi_{j, 5}=-2 \rho_{g}^{-2} h_{\zeta, j}+a_{j} \eta_{j}+E_{j, 3} \quad \text { in } \Omega_{i}
$$


where $E_{j, 3}:=E_{j, 1}+\left[\mathcal{L}_{\chi}, \psi[1,2] \circ x\right]\left(\phi_{j, 3}+\phi_{j, 4}\right)$. If we let

$$
E:=-\sum\left(E_{j, 3}+\left[\mathcal{L}_{\chi}, \psi\left[\ell_{j}-2, \ell_{j}-1\right] \circ x\right] \phi_{j, 5}\right),
$$

then $\|E\|_{0} \leq C \ell \tau$. Let $\phi_{E}, \Theta_{E, j}, \Lambda_{E, i}, \bar{\Lambda}_{E, m}$ be given by proposition 4.2 .8 with the above $E$, and define

$$
\begin{array}{r}
\phi_{\zeta}:=\sum \phi_{j, 5} \psi\left[\ell_{j}-2, \ell_{j}-1\right] \circ x+\phi_{E}, \\
\Theta_{\zeta, j}:=a_{j}+\Theta_{E, j}, \quad \Lambda_{\zeta, i}:=\Lambda_{E, i}, \quad \bar{\Lambda}_{\zeta, m}:=\bar{\Lambda}_{E, m} .
\end{array}
$$

Lemma 4.3.3. For any parameter $\left(\left(\zeta_{j}\right),\left(\vec{\xi}_{\Omega_{i}}\right),\left(\vec{\xi}_{\mathcal{C}_{m}}\right)\right) \in Z_{\gamma_{1}} \times \Xi_{\gamma_{2}} \times \bar{\Xi}_{\gamma_{2}}$, there are $\phi_{\vec{\xi}} \in C_{\text {loc }}^{2, \alpha}(M, \chi), \Theta_{\vec{\xi}, j} \in \mathbb{R}, \Lambda_{\vec{\xi}, i} \in \mathbb{R}^{3}, \bar{\Lambda}_{\vec{\xi}, m} \in \mathbb{R}^{3}$, unique by the construction, such that

$$
\begin{aligned}
& \text { 1. } \mathcal{L}_{\chi} \phi_{\vec{\xi}}=-2 \rho_{g}^{-2}\left(\sum h_{\vec{\xi}, \Omega_{i}}+\sum h_{\vec{\xi}, \mathcal{C}_{m}}\right)+\sum \Lambda_{\vec{\xi}, i} \cdot \omega_{i}+\sum \bar{\Lambda}_{\vec{\xi}, m} \cdot \bar{\omega}_{m}+ \\
& \sum \Theta_{\vec{\xi}, j} \eta_{j},
\end{aligned}
$$

2. $\left\|\phi_{\vec{\xi}}\right\|_{2} \leq C \gamma_{2} \ell^{2} \tau$,

3. $\left|\Theta_{\vec{\xi}, j}\right| \leq C \gamma_{2} \ell^{2} \tau, \quad\left|\vec{\xi}_{\Omega_{i}}-\Lambda_{\vec{\xi}, \Omega_{i}}\right| \leq \frac{2}{3} \gamma_{2} \ell^{2} \tau, \quad\left|\vec{\xi}_{\mathcal{C}_{m}}-\bar{\Lambda}_{\vec{\xi}, \mathcal{C}_{m}}\right| \leq$ $\frac{2}{3} \gamma_{2} \ell^{2} \tau^{(3-2 \lambda) / 2}$.

Proof. By lemma 4.2.6 and arguments similar to the proof of proposition 4.2 .9$, there are $\phi_{\vec{\xi}, i}, \Lambda_{\vec{\xi}, j}$ and $\bar{\phi}_{\vec{\xi}, m}, \bar{\Lambda}_{\vec{\xi}, m}$ such that

$$
\begin{gathered}
\mathcal{L}_{\chi} \phi_{\vec{\xi}, i}=-2 \rho_{g}^{-2} h_{\vec{\xi}, \Omega_{i}}+\Lambda_{\vec{\xi}, i} \cdot \omega_{i} \quad \text { in } \Omega_{i}, \quad \phi_{\vec{\xi}, i}=0 \quad \text { on } \partial \Omega_{i}, \\
\mathcal{L}_{\chi} \bar{\phi}_{\vec{\xi}, m}=-2 \rho_{g}^{-2} h_{\vec{\xi}, \mathcal{C}_{m}}+\bar{\Lambda}_{\vec{\xi}, m} \cdot \bar{\omega}_{m} \quad \text { in } \mathcal{C}_{m}, \quad \bar{\phi}_{\vec{\xi}, m}=0 \quad \text { on } \partial \mathcal{C}_{m}, \\
\left\|\phi_{\vec{\xi}, i}: C^{2, \alpha}\left(\Omega_{i}, \chi\right)\right\| \leq C \gamma_{2} \ell^{2} \tau, \quad\left\|\bar{\phi}_{\vec{\xi}, m}: C^{2, \alpha}\left(\mathcal{C}_{m}, \chi\right)\right\| \leq C \gamma_{2} \ell^{2} \tau^{(3-2 \lambda) / 2} .
\end{gathered}
$$

By the formula of lemma 4.2.6,

$$
\begin{aligned}
\vec{\xi}_{\Omega_{i}}-\Lambda_{\vec{\xi}, i} & =\vec{\xi}_{\Omega_{i}}-\int_{\Omega_{i}} h_{\vec{\xi}, \Omega_{i}} f_{i} d \mathbf{g} \cdot K_{i}^{-1} \\
& =\vec{\xi}_{\Omega_{i}}-\int_{\Omega_{i}} h_{\vec{\xi}, \Omega_{i}} \vec{\nu}_{\zeta, \vec{\xi}} \cdot \vec{e} d \mathbf{g} \cdot K_{i}^{-1}+\int_{\Omega_{i}} h_{\vec{\xi}, \Omega_{i}}\left(\vec{\nu}_{\zeta, \vec{\xi}} \cdot \vec{e}-f_{i}\right) d \mathbf{g} \cdot K_{i}^{-1}
\end{aligned}
$$


where $f_{i}=\left(f_{i, 1}, f_{i, 2}, f_{i, 3}\right), \quad \vec{e}=\left(\vec{e}_{1}, \vec{e}_{2}, \vec{e}_{3}\right)$. Since $\int_{\Omega_{i}} h_{\vec{\xi}, \Omega_{i}} \vec{\nu}_{\zeta, \vec{\xi}} \cdot \vec{e} d \mathbf{g}=\vec{\xi}_{\Omega_{i}}$ by the balancing formula [S2][KKS], we obtain, by lemmas $4.2 .3,4.2 .5$ and choosing small enough $\delta$ if necessary,

$$
\left|\vec{\xi}_{\Omega_{i}}-\Lambda_{\vec{\xi}, i}\right| \leq \tilde{\varepsilon} \gamma_{2} \ell^{2} \tau, \quad \text { and similarly } \quad\left|\vec{\xi}_{\mathcal{C}_{m}}-\bar{\Lambda}_{\vec{\xi}, \mathcal{C}_{m}}\right| \leq \tilde{\varepsilon} \gamma_{2} \ell^{2} \tau^{(3-2 \lambda) / 2} .
$$

Let $a_{j}$ be given by proposition 4.1 .4 with $\phi=\phi_{\vec{\xi}, i}$ restricted on $V_{j}\left[\ell_{j} / 2, \ell_{j}\right] \subset$ $\Omega_{i}$. Then,

$$
\left|a_{j}\right| \leq C \gamma_{2} \ell^{2} \tau, \quad\left\|\left(\phi_{\vec{\xi}, i}+\sum a_{j} v_{j}\right) \psi_{\Omega_{i}}\right\|_{2} \leq C \gamma_{2} \ell^{2} \tau .
$$

where $\psi_{\Omega_{i}}$ is the smooth cutoff function defined in the proof of lemma 4.2.9. Let $\psi_{\mathcal{C}_{m}}$ be also the cutoff function from lemma 4.2.9 and define

$$
E:=-\sum\left[\mathcal{L}_{\chi}, \psi_{\Omega_{i}}\right]\left(\phi_{\vec{\xi}, i}+\sum a_{j} v_{j}\right)-\sum\left[\mathcal{L}_{\chi}, \psi_{\mathcal{C}_{m}}\right] \bar{\phi}_{\vec{\xi}, m}
$$

Then, $\|E\|_{0} \leq C \gamma_{2} \ell^{2} \tau^{1+\lambda / 2}$. Let $\phi_{E}, \Lambda_{E, i}, \bar{\Lambda}_{E, m}, \Theta_{E, j}$ be given by proposition 4.2 .9 with the above $E$, and define

$$
\begin{gathered}
\phi_{\xi}:=\sum \psi_{\Omega_{i}}\left(\phi_{\vec{\xi}, i}+\sum a_{j} v_{j}\right)+\sum \psi_{\mathcal{C}_{m}} \bar{\phi}_{\vec{\xi}, \mathcal{C}_{m}}+\phi_{E} \\
\Lambda_{\vec{\xi}, i}:=\Lambda_{\vec{\xi}, i}+\Lambda_{E, i}, \quad \bar{\Lambda}_{\vec{\xi}, m}:=\bar{\Lambda}_{\vec{\xi}, m}+\bar{\Lambda}_{E, m}, \quad \Theta_{\vec{\xi}, j}:=a_{j}+\Theta_{E, j} .
\end{gathered}
$$

Proposition 4.3.4. For any parameter

$$
\left(\left(\zeta_{j}\right),\left(\vec{\xi}_{\Omega_{i}}\right),\left(\vec{\xi}_{\mathcal{C}_{m}}\right)\right) \in Z_{\gamma_{1}} \times \Xi_{\gamma_{2}} \times \bar{\Xi}_{\gamma_{2}}
$$

there are $\Phi_{\zeta, \vec{\xi}} \in C_{\text {loc }}^{2, \alpha}(M, \chi), \Theta_{\zeta, \vec{\xi}, j} \in \mathbb{R}, \Lambda_{\zeta, \vec{\xi}, i} \in \mathbb{R}^{3}, \bar{\Lambda}_{\zeta, \vec{\xi}, m} \in \mathbb{R}^{3}$, unique by the construction, such that

1. $\mathcal{L}_{\chi} \Phi_{\zeta, \vec{\xi}}=-2 \rho_{g}^{-2} H+\sum \Theta_{\zeta, \vec{\xi}, j} \eta_{j}+\sum \Lambda_{\zeta, \vec{\xi}, i} \cdot \omega_{i}+\sum \bar{\Lambda}_{\zeta, \vec{\xi}, m} \cdot \bar{\omega}_{m} \quad$ in $M$,

2. $\left\|\Phi_{\zeta, \vec{\xi}}\right\|_{2} \leq C\left(\gamma_{1}, \gamma_{2}\right) \ell^{2} \tau$,

3. $\left|\zeta_{j}-\Theta_{\zeta, \vec{\xi}, j}\right| \leq \frac{3}{4} \gamma_{1} \ell^{2} \tau, \quad\left|\vec{\xi}_{\Omega_{i}}-\Lambda_{\zeta, \vec{\xi}, i}\right| \leq \frac{3}{4} \gamma_{2} \ell^{2} \tau, \quad\left|\vec{\xi}_{\mathcal{C}_{m}}-\bar{\Lambda}_{\zeta, \vec{\xi}, m}\right| \leq$ $\frac{3}{4} \gamma_{2} \ell^{2} \tau^{(3-2 \lambda) / 2}$. 
Proof. Define

$$
\begin{gathered}
\Phi_{\zeta, \vec{\xi}}:=\phi_{\tau}+\phi_{\zeta}+\phi_{\vec{\xi}}, \quad \Theta_{\zeta, \vec{\xi}, j}:=\Theta_{\tau, j}+\Theta_{\zeta, j}+\Theta_{\vec{\xi}, j}, \\
\Lambda_{\zeta, \vec{\xi}, \phi, i}:=\Lambda_{\tau, i}+\Lambda_{\zeta, i}+\Lambda_{\vec{\xi}, i}, \quad \bar{\Lambda}_{\zeta, \vec{\xi}, m}:=\bar{\Lambda}_{\tau, m}+\bar{\Lambda}_{\zeta, m}+\bar{\Lambda}_{\vec{\xi}, m}
\end{gathered}
$$

where all $\phi$ 's, $\Theta$ 's, $\Lambda$ 's, $\bar{\Lambda}$ 's are from the previous three lemmas. (We take large enough $\gamma_{2}$ in terms of $C$, large enough $\gamma_{1}$ in terms of $C, \gamma_{2}$, and large enough $\ell$ in terms of $C, \gamma_{1}, \gamma_{2}$ so that our claim holds.)

\section{Nonlinear Term and the Main Theorem.}

\subsection{Nonlinear term.}

For a function $\phi$ on $M$ such that $\vec{X}_{\zeta, \vec{\xi}, \phi}:=\vec{X}_{\zeta, \vec{\xi}}+\phi \vec{\nu}_{\zeta, \vec{\xi}}$ is an immersion, the nonlinear term $Q_{\zeta, \vec{\xi}, \phi}$ is defined from the mean curvature identity (1.1)

$$
Q_{\zeta, \vec{\xi}, \phi}:=\rho_{g}^{-2} H_{\zeta, \vec{\xi}, \phi}-\rho_{g}^{-2} H_{\zeta, \vec{\xi}}-\frac{1}{2} \mathcal{L}_{\chi} \phi
$$

where $H_{\zeta, \vec{\xi}}, H_{\zeta, \vec{\xi}, \phi}$ are mean curvature functions related to $\vec{X}_{\zeta, \vec{\xi}}, \vec{X}_{\zeta, \vec{\xi}, \phi}$, respectively. As in [K1], a pointwise rescaling $T_{\chi}$ of a tensor $T$ is defined by $\left|T_{\chi}\right|_{\chi}=|T|$ where the length in the left is with respect to $\chi$ and in the right is with respect to $\mathbf{g}$.

Lemma 5.1.1. If $\phi \in C_{l o c}^{2, \alpha}(M, \chi)$ and $|\phi \mathbf{A}|<1$ on $M$, then $\vec{X}_{\zeta, \vec{\xi}, \phi}:=$ $\vec{X}_{\zeta, \vec{\xi}}+\phi \vec{\nu}_{\zeta, \vec{\xi}}$ is an immersion, and if in addition

$$
\left\|\phi \mathbf{A}_{\chi}: C^{0, \alpha}(M, \chi)\right\| \leq C \quad \text { and } \quad\left\|(\nabla \phi)_{\chi}: C^{0, \alpha}(M, \chi)\right\| \leq C,
$$

then

$$
\begin{aligned}
\left\|Q_{\zeta, \vec{\xi}, \phi}\right\|_{q} \leq C\left\{\left\|\rho_{g}^{-1} \mathbf{A}_{\chi}\right\|_{q_{1}}^{2} \cdot\|\phi\|_{q_{2}}+\left\|\rho_{g}^{-1} \mathbf{A}_{\chi}\right\|_{q_{3}} \cdot\|\nabla \phi\|_{q_{4}}\right. \\
\left.+\left\|\rho_{g}^{-2} \cdot(\nabla \mathbf{A})_{\chi}\right\|_{q_{5}} \cdot\|\phi\|_{q_{6}}+\left\|\nabla_{g}^{2} \phi\right\|_{q_{7}}\right\}\left\{\left\|\phi \mathbf{A}_{\chi}\right\|_{q_{8}}+\left\|(\nabla \phi)_{\chi}\right\|_{q_{8}}\right\}
\end{aligned}
$$

where $\|\cdot\|_{q}:=\left\|\cdot: C^{0, \alpha}(M, \chi, q)\right\|$ and $q, q_{1}, \cdots, q_{8}$ are arbitrary functions satisfying $q_{1}^{2} \cdot q_{2}=q_{3} \cdot q_{4}=q_{5} \cdot q_{6}=q_{7}, q_{7} \cdot q_{8}=q$.

Proof. follows from [K1, Appendix C] by similar arguments to the ones in $[\mathrm{K} 1, \mathrm{~V} .1 .3]$. 
Proposition 5.1.2. Suppose $\|\phi\|_{2} \leq \gamma_{3} \ell^{2} \tau$ where $\gamma_{3}$ is a constant to be determined in the proof of Theorem 5.2.1. Then, $\vec{X}_{\zeta, \vec{\xi}, \phi}:=\vec{X}_{\zeta, \vec{\xi}}+\phi \vec{\nu}_{\zeta, \vec{\xi}}$ is an immersion and

$$
\left\|Q_{\zeta, \vec{\xi}, \phi}\right\|_{0} \leq C \tau^{-(3-2 \lambda) / 4}\|\phi\|_{2}^{2} \leq C \gamma_{3}^{2} \tau^{5 / 4}
$$

Proof. We first note that

$$
\mathbf{A}_{\chi}=\rho_{g}^{2} \mathbf{A}, \quad(\nabla \mathbf{A})_{\chi}=\rho_{g}^{3} \nabla \mathbf{A}=\rho_{g}^{3}\left(\nabla_{\chi} \mathbf{A}+T * \mathbf{A}\right)
$$

where $T:=\nabla-\nabla_{\chi}$ is the difference tensor of the two connections and $*$ is the contraction. Since

$$
\left\|T: C^{1}(M, \chi)\right\| \leq C\left\|\rho_{g}^{2} \nabla \rho_{g}^{-2}: C^{1}(M, \chi)\right\| \leq C,
$$

from lemma 3.3.4, we obtain from lemmas 3.3.3, 3.3.4 that

$$
\left\|\mathbf{A}_{\chi}: C^{1}\left(M, \chi, q_{1}\right)\right\| \leq C
$$

where $q_{1}:=e^{-(2 n-k-1) x}$ on $V_{i, q}[0, \infty]$ when $q>1$ or $q=1$ and $V_{i, 1}[0, \infty]$ was originally an end of large or moderate amplitude or a catenoidal end, $:=e^{-(n-2 k-2) x}$ on $V_{i, 1}[0, \infty]$ when $V_{i, 1}[0, \infty]$ was originally an end of small amplitude (recall that $-n-1<k<-1$ in this case), $q_{1}:=1$ on $\left(\cup \Omega_{i}^{\prime}\right) \bigcup\left(\cup V_{j}\left[0, \frac{\ell_{j}}{2}\right]\right),:=e^{2\left(x-\ell_{j} / 2\right)}$ on $V_{j}\left[\frac{\ell_{j}}{2}, \ell_{j}\right],:=e^{\ell_{m}}$ on $\mathcal{C}_{m},:=e^{-2 x+\ell_{k}}$ on $\tilde{V}_{k}\left[\ell_{k}, \infty\right]$ and that

$$
\left\|(\nabla \mathbf{A})_{\chi}: C^{1}\left(M, \chi, q_{2}\right)\right\| \leq C
$$

where $q_{2}:=e^{-(3 n-k-1) x}$ on $V_{i, q}[0, \infty]$ when $q>1$ or $q=1$ and $V_{i, 1}[0, \infty]$ was originally an end of large or moderate amplitude or a catenoidal end, $:=e^{-(2 n-3 k-3) x}$ on $V_{i, 1}[0, \infty]$ when $V_{i, 1}[0, \infty]$ was originally an end of small amplitude, $q_{2}:=e^{x}$ on $\left(\cup \Omega_{i}^{\prime}\right) \bigcup\left(\cup V_{j}\left[0, \frac{\ell_{j}}{2}\right]\right),:=e^{3 x-\ell_{j}}$ on $V_{j}\left[\frac{\ell_{j}}{2}, \ell_{j}\right],:=e^{2 \ell_{m}}$ on $\mathcal{C}_{m}^{\prime},:=e^{-3 x+5 \ell_{k}}$ on $\tilde{V}_{k}\left[\ell_{k}, \infty\right]$.

When $q: M \rightarrow \mathbb{R}$ is an arbitrary function,

$$
\left\|(\nabla \phi)_{\chi}: C^{1}(M, \chi, q)\right\| \leq C\left\|\phi: C^{2, \alpha}(M, \chi, \tilde{q} q)\right\|,
$$

where $\tilde{q}:=e^{-x}$ on $M \backslash\left(\cup V_{i, q}[0, \infty] \bigcup \cup \tilde{V}_{k}\left[\ell_{k}, \infty\right]\right),:=e^{(n-k-1) x}$ on $V_{i, q}[0, \infty]$, $e^{x-2 \ell_{k}}$ on $\tilde{V}_{k}\left[\ell_{k}, \infty\right]$, whose proof follows from $(\nabla \phi)_{\chi}=\rho_{g} \nabla \phi$ and lemma 3.3.4. Since $\nabla_{g}^{2} \phi=\nabla_{\chi}^{2} \phi+T * \nabla \phi$,

$$
\left\|\nabla_{g}^{2} \phi: C^{1}(M, \chi, q)\right\| \leq C\left\|\phi: C^{2, \alpha}(M, \chi, q)\right\| .
$$


From lemma 5.1.1 and the above estimates, we get for any arbitrary function $q: M \rightarrow \mathbb{R}$

$$
\left\|Q_{\zeta, \vec{\xi}, \phi}: C^{0, \alpha}\left(M, \chi, \bar{q} q^{2}\right)\right\| \leq C\left\|\phi: C^{2, \alpha}(M, \chi, q)\right\|^{2}
$$

where $\bar{q}:=e^{x}$ on $M \backslash\left(\cup V_{i, q}[0, \infty] \bigcup \cup \tilde{V}_{k}\left[\ell_{k}, \infty\right]\right),:=e^{-(n-k-1) x}$ on $V_{i, q}[0, \infty]$, $:=e^{-x+2 \ell_{k}}$ on $\tilde{V}_{k}\left[\ell_{k}, \infty\right]$. The result follows.

\subsection{Proof of the main theorem.}

Theorem 5.2.1. If $\tau$ is small enough, then there are

$$
\left(\left(\zeta_{j}\right),\left(\vec{\xi}_{\Omega_{i}}\right),\left(\vec{\xi}_{\mathcal{C}_{m}}\right)\right) \in Z_{\gamma_{1}} \times \Xi_{\gamma_{2}} \times \bar{\Xi}_{\gamma_{2}}
$$

and $\varphi \in C^{\infty}(M)$ with small \|\|$_{2}$ norm such that $\vec{X}_{\zeta, \vec{\xi}, \varphi}: M \rightarrow \mathbb{E}^{3}$ given by $\vec{X}_{\zeta, \vec{\xi}, \varphi}:=\vec{X}_{\zeta, \vec{\xi}}+\varphi \vec{\nu}_{\zeta, \vec{\xi}}$ is a minimal immersion.

Proof. Fix $\alpha^{\prime}$ in $(0, \alpha)$ and define

$$
\mathcal{B}_{\gamma_{3}}:=\left\{\phi \in C_{l o c}^{2, \alpha^{\prime}}\left(M, \chi_{0, \overrightarrow{0}}\right):\left\|\phi: C^{2, \alpha}\left(M, \chi_{0, \overrightarrow{0}}, \tilde{q}\right)\right\| \leq \gamma_{3} \ell^{2} \tau\right\},
$$

where $\tilde{q}$ is the function in definition 4.2 .8 and $\gamma_{3}$ is a constant to be determined in the middle of this proof. Because of $0<\alpha^{\prime}<\alpha<1$ and the behavior of norms at ends, $\mathcal{B}_{\gamma_{3}}$ is compact in

$$
\mathcal{X}:=\left\{\phi \in C_{\text {loc }}^{2, \alpha^{\prime}}\left(M, \chi_{0, \overrightarrow{0}}\right):\left\|\phi: C^{2, \alpha^{\prime}}\left(M, \chi_{0, \overrightarrow{0}}, \bar{q}\right)\right\|<\infty\right\},
$$

where $\bar{q}:=e^{\lambda^{\prime} x}$ on $\cup V_{i, q}[0, \infty],:=e^{\lambda^{\prime} x} e^{-(1-2 \lambda) \ell_{k} / 2}$ on $\tilde{V}_{k}\left[\ell_{k}, \infty\right],:=\tilde{q}$ on $M \backslash\left(\cup V_{i, q}[0, \infty] \cup \cup \tilde{V}_{k}\left[\ell_{k}, \infty\right]\right)$ for a small positive constant $\lambda^{\prime}$. So, $Z_{\gamma_{1}} \times$ $\Xi_{\gamma_{2}} \times \bar{\Xi}_{\gamma_{2}} \times \mathcal{B}_{\gamma_{3}}$ is a compact convex subset of the Banach space $\mathbb{R}^{K+2 I} \times$ $\mathbb{R}^{3(I+1)} \times \mathbb{R}^{3(K+I)} \times \mathcal{X}$.

Now, consider the map

$$
\mathcal{J}: Z_{\gamma_{1}} \times \Xi_{\gamma_{2}} \times \bar{\Xi}_{\gamma_{2}} \times \mathcal{B}_{\gamma_{3}} \rightarrow \mathbb{R}^{K+2 I} \times \mathbb{R}^{3(I+1)} \times \mathbb{R}^{3(K+I)} \times \mathcal{X}
$$

which sends $\left(\left(\zeta_{j}\right),\left(\vec{\xi}_{\Omega_{i}}\right),\left(\vec{\xi}_{\mathcal{C}_{m}}\right), \phi\right)$ to

$$
\begin{aligned}
\left(\left(\zeta_{j}-\Theta_{\zeta, \vec{\xi}, j}-\Theta_{\zeta, \vec{\xi}, \phi, j}\right),\left(\vec{\xi}_{\Omega_{i}}-\Lambda_{\zeta, \vec{\xi}, i}-\Lambda_{\zeta, \vec{\xi}, \phi, i}\right)\right. & \\
& \left.\left(\vec{\xi}_{\mathcal{C}_{m}}-\bar{\Lambda}_{\zeta, \vec{\xi}, m}-\bar{\Lambda}_{\zeta, \vec{\xi}, \phi, m}\right), \Phi_{\zeta, \vec{\xi}}+\varphi_{\zeta, \vec{\xi}, \phi}\right) .
\end{aligned}
$$


where

$$
\Theta_{\zeta, \vec{\xi}, j}, \Lambda_{\zeta, \vec{\xi}, i}, \bar{\Lambda}_{\zeta, \vec{\xi}, m}, \Phi_{\zeta, \vec{\xi}}
$$

are the ones in proposition 4.3.4, and

$$
\Theta_{\zeta, \vec{\xi}, \phi, j}, \Lambda_{\zeta, \vec{\xi}, \phi, i}, \bar{\Lambda}_{\zeta, \vec{\xi}, \phi, m}, \varphi_{\zeta, \vec{\xi}, \phi}
$$

are the ones in proposition 4.2 .9 with $E=Q_{\zeta, \vec{\xi}, \phi}$. By reviewing the construction, it is not hard to see that $\mathcal{J}$ is continuous and that

$$
\begin{gathered}
\left|\zeta_{j}-\Theta_{\zeta, \vec{\xi}, j}-\Theta_{\zeta, \vec{\xi}, \phi, j}\right| \leq \frac{3}{4} \gamma_{1} \ell^{2} \tau+C \gamma_{3}^{2} \tau^{5 / 4}, \\
\left|\vec{\xi}_{\Omega_{i}}-\Lambda_{\zeta, \vec{\xi}, i}-\Lambda_{\zeta, \vec{\xi}, \phi, i}\right| \leq \frac{3}{4} \gamma_{2} \ell^{2} \tau+C \gamma_{3}^{2} \tau^{5 / 4}, \\
\left|\vec{\xi}_{\mathcal{C}_{m}}-\bar{\Lambda}_{\zeta, \vec{\xi}, m}-\bar{\Lambda}_{\zeta, \vec{\xi}, \phi, m}\right| \leq \frac{3}{4} \gamma_{2} \ell^{2} \tau^{(3-2 \lambda) / 2}+C \gamma_{3}^{2} \tau^{7 / 4-\lambda}, \\
\left\|\Phi_{\zeta, \vec{\xi}}+\varphi_{\zeta, \vec{\xi}, \phi}: C^{2, \alpha}\left(M, \chi_{0, \overrightarrow{0}}, \tilde{q}\right)\right\| \leq C \ell^{2} \tau+C \gamma_{3}^{2} \tau^{5 / 4}
\end{gathered}
$$

So, by taking $\tau$ small enough and $\gamma_{3}$ large enough, we see that $\mathcal{J}$ sends $Z_{\gamma_{1}} \times \Xi_{\gamma_{2}} \times \bar{\Xi}_{\gamma_{2}} \times \mathcal{B}_{\gamma_{3}}$ into itself. Then, by the Schauder fixed point theorem [GT, Theorem 11.1], there is a fixed point, say $\left(\left(\zeta_{j}\right),\left(\vec{\xi}_{\Omega_{i}}\right),\left(\vec{\xi}_{\mathcal{C}_{m}}\right), \phi\right)$ of $\mathcal{J}$. By the definition of $\mathcal{J}$, we have

$$
\begin{aligned}
& \Theta_{\zeta, \vec{\xi}_{, j}}+\Theta_{\zeta, \vec{\xi}, \phi, j}=0, \quad \Lambda_{\zeta, \vec{\xi}_{, i}}+\Lambda_{\zeta, \vec{\xi}, \phi, i}=\overrightarrow{0} \\
& \bar{\Lambda}_{\zeta, \vec{\xi}, m}+\bar{\Lambda}_{\zeta, \vec{\xi}, \phi, m}=\overrightarrow{0}, \quad \underline{\phi}=\Phi_{\zeta, \vec{\xi}}+\varphi_{\zeta, \vec{\xi}, \phi}
\end{aligned}
$$

which immediately imply

$$
\mathcal{L}_{\chi}(\underline{\phi})=-2 \rho_{g}^{-2} H_{\zeta, \xi}-2 Q_{\zeta, \vec{\xi}}(\underline{\phi}) \quad \text { on } M
$$

Hence, $\vec{X}_{\zeta, \vec{\xi}, \underline{\varphi}}: M \rightarrow \mathbb{E}^{3}$ is a minimal immersion. $C^{\infty}$ follows from standard theory.

\section{References.}

[A] T. Aubin, Nonlinear analysis on Manifolds. Monge-Ampère Equations, Springer Verlag, 1982.

[C] I. Chavel, Eigenvalues in Riemannian Geometry, Academic Press, 1984. 
[Ch] J. Choe, Index,vision number, and stability of complete minimal surfaces, Arch. Rational Mech. Anal. 109 (1990), 195-212.

[CR] C. Cosin and A. Ros, A Plateau problem at infinity for properly immersed minimal surfaces with finite total curvature, preprint.

[DHKW] U. Dierkes, S. Hildebrandt, A. Küster, and O. Wohlrab, Minimal Surfaces, vol I., Springer Verlag, 1992.

[EK] N. Ejiri and M. Kotani, Index and flat ends of minimal surfaces, Tokyo J. Math. 16 (1993), 37-48.

[FP] S. Fakhi and F. Pacard, Existence results for minimal hypersurfaces with finite total curvature, to appear, Manuscripta Mathematica.

[G] R. Gulliver, Index and total curvature of complete minimal surfaces, Proc. Symp. Pure Math. 44 (1986), 207-212

[GT] D. Gilbarg and N.S. Trudinger, Elliptic partial differential equations of second order, 2nd Edition, Springer-Verlag, 1983.

[HK] D. Hoffman and H. Karcher, Complete embedded minimal surfaces of finite total curvature, Encyclopædia of Mathematical Sciences, 90, Springer Verlag, 1997, 5-93.

[JM] L. Jorge and W. H. Meeks III, The topology of complete minimal surfaces of finite total Gaussian curvature, Topology 22 (1983), 203-221.

[K1] N. Kapouleas, Complete constant mean curvature surfaces in Euclidean three space, Ann. of Math. 131 (1990), 239-330.

[K2] Constant mean curvature surfaces constructed by fusing Wente tori, Proc. Natl. Acad. Sci. USA 89 (1992), 5695-5698.

[K3] Constant mean curvature surfaces in Euclidean spaces, Proceedings of the International Congress of Mathematicians, Zürich, Switzerland 1994(1995), Birkhäuser, 481-490.

[K4] Constant mean curvature surfaces constructed by fusing Wente tori, Invent. Math. 119 (1995), 443-518.

[K5] Complete embedded minimal surfaces of finite total curvature, J. of Differential Geometry 47 (1997), 95-169.

[KKS] N. Korevaar, R. Kusner, and B. Solomon, The structure of complete embedded surfaces with constant mean curvature, J. of Differential Geometry 30 (1989), 465-503. 
[MPU] R. Mazzeo, D. Pollack, and K. Ulenbeck, Connected sum constructions for constant scalar curvature metrics, Topol. Methods Nonlinear Anal. 6 (1995), 207-233.

[MP] R. Mazzeo and F. Pacard, Constant mean curvature surfaces with Delaunay ends, to appear, Comm. Anal. Geom.

[MPP] R. Mazzeo, F. Pacard, and D. Pollack, Connected sums of constant mean curvature surfaces in Euclidean 3 space, to appear, Journal für die Reine und Angewandte Mathematik.

[MR] S. Montiel and A. Ros, Schrödinger operators associated to a holomorphic map, volume 1481 of Lecture notes in mathematics, pages 147-174, Springer Verlag, 1990.

[N] J. C. C. Nitsche, Lectures on minimal surfaces, vol 1, Cambridge University Press, 1989 .

[O] R. Osserman, A survey of minimal surfaces, Dover Publications, New York, 2nd edition, 1986.

[P] J. Pérez, On Singly-Periodic Minimal Surfaces with Planar ends, Trans. Amer. Math. Soc. 349 (1997), 2371-2389.

[PR] J. Pérez and A. Ros, The space of properly embedded minimal surfaces with finite total curvature, Indiana Univ. Math J.45 (1996), 177-204.

[RT] H. Rosenberg and E. Toubiana, Some remarks on deformations of minimal surfaces, Trans. Amer. Math. Soc. 295 (1986), 491-499.

[S1] R. Schoen, Uniqueness, symmetry, and embeddedness of minimal surfaces, J. Differential Geometry 18 (1983), 791-809.

[S2] R. Schoen, The existence of weak solutions with prescribed singular behavior for a conformally invariant scalar equation, Comm. Pure Appl. Math. 41 (1988), 317-392

[Sm] N. Smale, $A$ bridge principle for minimal and constant mean curvature submanifolds in $\mathbb{R}^{N}$, Invent. Math. 90 (1987), 505-549.

[T] J. Tysk, Eigenvalue estimates with applications to minimal surfaces, Pacific J. of Math. 128 (1987), 361-366.

[Y] S.D. Yang, On a connected sum construction for complete minimal surfaces of finite total curvature, $\mathrm{PhD}$ thesis (1997), Brown University. 
A connected sum construction for complete minimal surfaces

ReCEIVED JANUARY 22, 1999.

BROWN UNIVERSITY

PROVIDENCE, RI 02912

E-mail address: sdyang@math.brown.edu 